\title{
Peripheral MR angiography : costs, effects and technical improvements
}

Citation for published version (APA):

de Vries, M. (2005). Peripheral MR angiography : costs, effects and technical improvements. [Doctoral Thesis, Maastricht University]. Universitaire Pers Maastricht. https://doi.org/10.26481/dis.20051209mv

Document status and date:

Published: 01/01/2005

DOI:

10.26481/dis.20051209mv

Document Version:

Publisher's PDF, also known as Version of record

\section{Please check the document version of this publication:}

- A submitted manuscript is the version of the article upon submission and before peer-review. There can be important differences between the submitted version and the official published version of record.

People interested in the research are advised to contact the author for the final version of the publication, or visit the DOI to the publisher's website.

- The final author version and the galley proof are versions of the publication after peer review.

- The final published version features the final layout of the paper including the volume, issue and page numbers.

Link to publication

\footnotetext{
General rights rights.

- You may freely distribute the URL identifying the publication in the public portal. please follow below link for the End User Agreement:

www.umlib.nl/taverne-license

Take down policy

If you believe that this document breaches copyright please contact us at:

repository@maastrichtuniversity.nl

providing details and we will investigate your claim.
}

Copyright and moral rights for the publications made accessible in the public portal are retained by the authors and/or other copyright owners and it is a condition of accessing publications that users recognise and abide by the legal requirements associated with these

- Users may download and print one copy of any publication from the public portal for the purpose of private study or research.

- You may not further distribute the material or use it for any profit-making activity or commercial gain

If the publication is distributed under the terms of Article $25 \mathrm{fa}$ of the Dutch Copyright Act, indicated by the "Taverne" license above, 


\section{Peripheral MR Angiography \\ Costs, effects and technical improvements}


(C) M. de Vries, Maastricht 2005

mdvr@rdia.azm.nl

ISBN 9052784973

Printed by: Datawyse / Universitaire Pers Maastricht

Cover design: "Satellite image of the Mississippi River Delta"

Courtesy to NASA Earth Observatory and Suzanne Gerretsen.

Financial support for publication of this thesis was generously provided by: Phillips Medical Systems, Schering AG, Medis Medical Imaging Systems Bv, GE Healthcare, Guerbet, and the Netherlands Heart Foundation. 


\title{
Peripheral MR Angiography \\ Costs, effects and technical improvements
}

\author{
Proefschrift
}

ter verkrijging van de graad van doctor aan de Universiteit Maastricht, op gezag van de Rector Magnificus

Prof. mr. G.P.M.F. Mols, volgens het besluit van het College van Decanen,

in het openbaar te verdedigen

op vrijdag 9 december 2005 om 12.00 uur

door

Marianne de Vries

geboren op 21 juni 1976 te Groningen

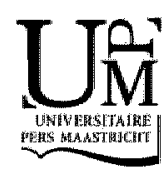




\section{Promotores:}

Prơf. dr. J.M.A. van Engelshoven

Prof. dr. M.G.M. Hunink (Erasmus Universiteit Rotterdam)

Coipromotores

Dr. M.W. de Haan

Dr. P.J. Nelemans

\section{Beoordelingscommissie}

Prof. dr. P.J.E.H.M. Kitslaar, voorzitter

Prof. dr. D.A. Legemate (Universiteit van Amsterdam)

Prof. dir. J.G. Maessen

Prof. dr. J.L. Severens 


\section{Contents}

\section{Chapter 1}

General Introduction

Chapter 2

Contrast-Enhanced Peripheral MR Angiography using SENSE in multiple stations: Feasibility study

\section{Chapter 3}

Comparison of generic and disease-specific questionnaires for the assessment of quality-of-life in patients with peripheral arterial disease

Chapter 4

Clinical and cost comparisons of duplex ultrasound and contrastenhanced magnetic resonance angiography in patients with peripheral arterial disease: a multicenter randomized trial

Chapter 5

Multicenter randomized controlled trial of the costs and effects of non-Invasive diagnostic imaging in patients with peripheral disease: The DIPAD trial

Chapter 6

Contrast-enhanced peripheral MR angiography at 3.0 Tesla: Initial experience with a whole-body scanner in healthy volunteers

Chapter 7

Accuracy of semi-automated analysis of 3D contrast-enhanced MR angiography for detection and quantification of aortoiliac stenoses

Chapter 8

General discussion

Chapter 9

Summary and conclusions

Nederlandse samenvatting

Appendix

Dankwoord

Curriculum Vitae 



\section{Chapter 1}

Introduction 


\section{Introduction}

\section{Peripheral arterial disease}

Peripheral arterial disease (PAD) of the lower extremities is a common and disabling disease $(1,2)$. PAD originates from arteriosclerosis that causes stenoses in the arteries of the lower extremities. The first manifestation of symptomatic PAD is usually intermittent claudication. Patients with intermittent claudication have a decrease in walking distance due to pain complaints in their legs, resulting in major changes in lifestyle and quality-of-life. In a minority of the patients PAD progresses to criticall limb ischemia, leading to rest pain and /or tissue necrosis in the lower legs.

\section{Imaging workup}

Revascularisation of the stenotic arteries is often successfully performed by means of percutaneous or surgical interventions. Imaging workup is necessary to determine the optimal revascularisation strategy in order to improve quality-of-life for claudication patients, or to avoid tissue loss in patients with critical ischemia. When revascullarisation is being considered, most centers in the Netherlands use duplex ultrasound (DUS) as the initial imaging modality, sometimes followed by digital subtraction angiography (DSA) $(3,4)$. Only a few centers use contrast-enhanced MR angiography (CE-MRA) or computed tomographic angiography (CTA) as the initial imaging modality for detecting stenoses in patients with $P A D$.

DUS is a well-established non-invasive modality that provides both anatomical and functional information about the arterial system, resulting in an accurate determination of the stenosis severity (5-7). However, DUS is operator-dependent, and does not provide an easy-to-interpret image ("roadmap") of the vascular system. Moreover, depiction of the crural arteries with DUS is often difficult and time consuming.

CTA and CE-MRA are both relatively new, non-invasive tests with high accuracy for the detection of stenoses in patients with PAD (8-12). Both techniques provide three-dimensional images of the arterial system. Moreover, CTA and CE-MRA depict the upper and lower leg arteries routinely, thereby producing a "roadmap" of the arteries, which is very useful for treatment planning.

Disadvantages of CE-MRA include the injection of a contrast agent, and the fact that some patients are claustrophobic or have other contraindications to undergo MR imaging (13).

Disadvantages of CTA are the use of radiation and potentially nephrotoxic lodinated contrast agents (14). In addition, vessel wall calcifications can affect image interpretation and the $3 \mathrm{D}$ reconstruction techniques are time-consuming.

In the everyday imaging work-up of patients with PAD the question arises which imaging test is optimal for treatment planning, and at the same time, is the most cost-effective. Addressing both issues we have optimized the CE-MRA protocol for patients with PAD, and performed a multicenter cost-effectiveness study: the DIPAD study. 


\section{DIPAD study}

The Diagnostic Imaging for Peripheral Arterial Disease (DIPAD) study was performed at 4 hospitals in the Netherlands: Maastricht University Medical Center; Erasmus Medical Center Rotterdam, St Catharina Hospital Eindhoven and the University Medical Center St Radboud. In this prospective study all patients with PAD who were scheduled for initial diagnostic imaging were randomly assigned to undergo CE-MRA or the currently employed test, which was CTA in Rotterdam and DUS in the other 3 centers.

To assess cost-effectiveness, all costs of (additional) imaging, therapeutic interventions and outpatient visits were collected during a 6 months follow-up period. Other outcome measures included change in quality-of-life, physician's confidence to take a treatment decision, and change in disease severity.

Objectives of this thesis

1. To optimize current contrast-enhanced MR angiographic protocols for patients with PAD.

2. To assess whether disease-specific or generic questionnaires are preferable to assess quality-of-life after treatment of patients with PAD (the DIPAD study).

3. To determine the optimal non-invasive initial imaging workup for patients with $P A D$, which is based on the clinical and economic consequences of replacing DUS by CE-MRA and of replacing CTA by CE-MRA (the DIPAD study).

4. To assess the future directions for CE-MRA such as the use of higher magnetic field strength, and optimisation of the image evaluation using a software program that semi-automated detects stenoses on 3D CE-MRA data.

\section{Outline of this thesis}

1. In chapter 2, the use of a parallel imaging technique (SENSE) in multiple stations to optimize peripheral CE-MRA is evaluated, using the IA-DSA as standard of reference.

2. In chapter 3, we determine which questioninaire is most sensitive to detect changes in quality-of-life after treatment for patients with PAD (the DIPAD study).

3. In chapter 4, the cost-effectiveness of DUS and CE-MRA as initial imaging test for patients with PAD is compared; based on these results it is recommended which imaging test is preferable (the DIPAD study).

4. In chapter 5, the results of the cost-effectiveness of CE-MRA versus DUS are combined with the results of cost-effectiveness of CE-MRA versus CTA (the DIPAD study).

5. In chapter 6, the feasibility of using the 3.0 Tesla scanner for peripheral CE-MRA is described.

6. In chapter 7, the accuracy of stenosis measurements on CE-MRA data of aortoiliac arteries using a semi-automated software program is assessed and compared with the accuracy of conventional stenosis measurements. 
7. Chapter 8 provides a general discussion, in which current and future imaging strategies for patients with PAD are discussed and recommended.

8. Chapter 9 provides a summary of the most important findings.

The research was funded by a grant of the Netherlands Organization for Health Research and Development. 


\section{References}

1. Meijer WT, Hoes AW, Rutgers D, Bots ML, Hotman A, Grobbee DE. Peripheral arterial disease in the elderly: The Rotterdam Study. Artenioscler Thromb Vasc Biol 1998:18(2):185-192.

2. Fowkes $F G$, Howsley $E$, Cawood EH, Macintyre $C C$, Ruckley $\mathrm{CV}$, Prescott RJ. Edinburgh Artery Study: prevalence of asymptomatic and symptomatic peripheral arterial disease in the general population. Int J Epidemial 1991;20(2):384-392.

3. Elsman BH, Legemate DA, van der Heyden FW, de Vos $H_{3}$ Mali WP, Eikelboom BC. The use of colar-coded duplex scanning in the selection of patients with lower extremity arterial disease for percutaneous transluminal angioplasty: a prospective study. Cardiovasc Intervent Radiol 1996;19(5):313-316.

4. Elsman $\mathrm{BH}$, Legemate $\mathrm{DA}$, van der Heijden $\mathrm{FH}$, de Vos $\mathrm{HJ}$ "Mali WP, Eikelboom BC. Impact of ultrasonographic duplex scanning on therapeutic decision making in lower-limb arterial disease. Bir J Surg 1995;82(5);630-633.

5. Koelemay MJ, den Hartog D, Prins MH, Kromhout JG, Legemate DA, Jacobs MJ. Diagnosis of arterial disease of the lower extremities with duplex ultrasonography. Br J Surg 1996;83(3):404409 .

6. Visser K, Hunink MG. Peripheral arterial disease: gadolinilum-enhanced MR angiography versus color"-guided duplex US-a meta-analysis. Radiology 2000;216(1):67-77.

7. de Vries SO, Hunink MG, Polak JF. Summary receiver operating characteristic curves as a technique for meta-analysis of the diagnostic performance of duplex ultrasonography in peripheral arterial disease. Acad Radiol 1996;3(4):361-369.

8. Martin ML, Tay KH, Flak $B$, et al. Multidetector CT angiography of the aortoiliac system and lower extremities: a prospective comparison with digital subtraction angiography. AJR Am J Roentgenol 2003;180(4):1085-1091.

9. Willmann JK, Wildermuth $S$, Pfammatter $T_{n}$ et al. Aortoiliac and renal arteries: prospective intraindividual comparison of contrast-enhanced three-dimensional MR angiography and multidetector row CT angiography. Radiology 2003;226(3):798-811.

10. Catalano $C_{r}$ Fraioli $F$, Laghi $A$, et al. Infrarenal aortic and lower-extremity arterial disease: diagnostic performance of multi-detector row CT angiography. Radiology 2004;231(2):555-563.

11. Koelemay MJ, Lijmer $J G$, Stoker $J$, Legemate DA, Bossuyt PM. Magnetic resonance angiography for the evaluation of lower extremity arterial disease: a metaranalysis. Jama 2001;285(10):1338-1345.

12. Nelemans PJ, Leiner $T$, de Vet $H C$, van Engelshoven JM. Peripheral arterial disease: metaanalysis of the diagnostic performance of MR angiography. Radiology $2000 ; 217(1): 105-114$.

13. Shellock FG, Crues JV. MR procedures: biologic effects, safety, and patient care. Radiology 2004:232(3):635-652.

14. Brenner DJ, Elliston CD. Estimated radiation risks potentially associated with full-body $\mathrm{CT}$ screening. Radiology 2004;232(3):735-738. 


\section{Chapter 2}

\section{Contrast-Enhanced Peripheral MR Angiography Using SENSE in Multiple Stations: Feasibility Study}

J Magn Reson Imaging (JMRI) 2005; 21:37-45

Marianne de Vries, Robbert J. Nijenhuis, Romhild M. Hoogeveen, Michiel W. de Haan, Jos M.A. van Engelshoven, Tim Leiner. 


\begin{abstract}
Purpose

To investigate if the use of parallel imaging is feasible and beneficial for peripheral contrast-enhanced magnetic resonance angiography (CE-MRA).
\end{abstract}

\title{
Materials and Methods
}

Nineteen consecutive patients underwent peripheral CE-MRA using SENSE with twofold reduction in the upper and lower leg stations. Conventional non-accelerated imaging using constant level appearance (CLEAR) was used in the aortoiliac station. The findings were compared with those in a similar patient group that underwent peripheral CE-MP angiography using our standard imaging protocol without SENSE. Intra-arterial digital subtraction angiography (IA-DSA) was used as the standard of reference. Lower extremity vessels were divided into anatomic segments (aortoiliac, upper legs, lower legs) for review. In each anatomic segment signal- and contrast to noise ratios (SNR, CNR), venous contamination, subjective image quality as well as sensitivity and specificity were determined for both patient groups.

Results

SNR and CNR improved significantly for the aortoiliac and upper leg segments (all Pvalues $\leq .001)$. Small reductions were seen in the frequency of disturbing venous enhancement $(P=N . S$.). There were no significant differences with regards to subjective image quality or diagnostic accuracy (all $P>3$ ). Overall sensitivity and specificity in the SENSE group were $81 \%$ and $95 \%$, respectivelly. For the nonSENSE group these figures were $79 \%$ and $96 \%$, respectively.

\section{Conclusions}

Preliminary results show that contrast-enhanced three-station peripheral MR angiography using a full length peripheral arterial coil in combination with SENSE in the upper and lower leg stations is feasible and useful for further optimization of peripheral MRA. Using SENSE allows for routine, high-quality depiction of the entire peripheral vascular tree including the pedal arch. Higher SENSE factors are needed for further optimization. 


\section{Introduction}

Contrast-enhanced peripheral MR angiography (CEMRA) is papidly becoming a widely accepted alternative to X-ray arteriography for the pre-interventional workup of peripheral arterial occlusive disease $(1-3)$. Using CE-MRA; the peripheral arterial tree can be depicted by acquiring three consecutive imaging volumes in the aortoiliac, upper leg and lower leg regions during injection of $0.1-0.3 \mathrm{mmol} / \mathrm{kg}$ of an extracellular MR contrast agent. A common problem when this sequential approach is used is sub optimal image quality of the lower legs $(4,5)$. Although not mandatory in every patient, depiction of the vessels in the lower leg is clinically important when considering treatment options such as distal bypass surgery in patients with severe intermittent claudication and chronic critical ischemia $(6,7)$. High resolution imaging of small, diseased lower leg arteries in these patients currently demands long imaging times, and is challenging because there is a direct trade-off between the duration of the acquisition and the chance for disturbing venous enhancement.

Recently, parallel imaging techniques such as sensitivity encoding (SENSE) have been introduced. SENSE allows for reduction of scan time by simultaneous collection of MR signals from multiple reception coils (8). When applied to CE-MRA of the peripheral arteries, SENSE allows for reduction in imaging time (and thus a decrease in the chance for venous enhancement) while maintaining spatial resolution. As was shown by Maki et al. and Kurihara et al., SENSE can be used for obtaining higher resolution images or more anatomical coverage in the same imaging time $(9,10)$. The inherent drawback of using parallel imaging is reduction in signal-to-noise ratio. However, signal loss may be reversed by faster gadolinium injection $(10,11)$.

The purpose of the current study was to investigate if the use of SENSE as part of a 'traditional' sequential multi-station imaging protocol could improve objective and subjective image quality by speeding up image acquisition, increasing spatial resolution and anatomical coverage. We compared our findings with those in a similar patient group that underwent peripheral CE-MRA using our standard imaging protocol without SENSE. In both groups of patients intra-arterial digital subtraction angiography (IA-DSA) was used as the standard of reference.

\section{Materials and Methods}

All acquisitions were performed on a $1.5 \mathrm{~T}$ clinical MR scanner (Intera, software release 8.2, Philips Medical Systems, Best, The Netherlands) with a gradient strength of $30 \mathrm{mT} / \mathrm{m}$ and a rise time of $200 \mu \mathrm{s}$. The study protocol was approved by the institutional review board, and all patients were required to give informed consent before they could be included into the study. 


\section{Study Design And Patients}

Thirty-eight consecutive patients, referred by their vascular surgeon for CE-MRA secondary to suspected or proven peripheral arterial disease, were included in the study. None of the patients had contra-indications for undergoing CE-MRA and IADSA, or declined to participate. All subjects underwent IA-DSA and CE-MRA within a period of 6 weeks. The first nineteen patients underwent CE-MRA using our standard protocol and the following nineteen patients underwent CE-MRA LISing SENSE. Patient characteristics and severity of disease are listed in table 1.

Table $1 \quad$ Patient characteristics

\begin{tabular}{lll}
\hline Imaging protocol & Non-SENSE & SENSE \\
\hline Number of patients & 19 & 19 \\
Sex (M/F) & $15 / 4$ & $12 / 7$ \\
Mean age (range $\pm \mathrm{SD})$ & $63(46-76 ; \pm 8.6)$ & $62(35-78 ; \pm 10.8)$ \\
Diabetes Mellitus & 5 & 4 \\
Prior peripheral vascullar surgery & 7 & 7 \\
Mild-moderate claudication & 13 & 13 \\
Severe claudication & 4 & 4 \\
Rest pain & 2 & 1 \\
Rest pain and ulcerations & 0 & 1 \\
\hline
\end{tabular}

SENSE: sensitivity encoding

\section{Standard CE-MRA Protocol}

The standard CE-MRA protocol consisted of four steps: 1) patient positioning, 2) acquisition of low-resolution time-of-flight (TOF) localizer scans, 3) acquisition of non contrast-enhanced three-dimensional gradient echo 'mask' scans (which were later subtracted from the corresponding contrast-enhanced images), and 4) acquisition of contrast-enhanced scans using identical imaging parameters as the mask scans.

TOF scout views were first acquired to outline aortoiliac, upper and lower limb arterial anatomy. TOF imaging parameters were identical for each station and were TR (ms) / TE (ms) / flip angle ( $)$ / acquisition time (s): 11/6.9/50/88. Orthogonal maximum intensity projections (MIPS) of the TOF datasets were generated to prescribe the subsequent 3D imaging volumes. Using these MIPS, 3D CE-MRA imaging volumes, taillored to individual patient anatomy in all three stations, were prescribed using the imaging parameters shown in table 2. Overlap between separate stations was set to $35 \mathrm{~mm}$. Appropriate start of CE-MRA acquisition was determined using real time bolus monitoring software (BolusTrak, Philips Medical Systems, Best, The Netherlands). When enhancement was first seen in the descending aorta, the patient was given a breath-hold command and image acquisition was started 4-5 sec later. K-space filling was set to linear for the aortoiliac arteries and to improved elliptical centric phase encoding (Contrast Enhanced Timing Robust Angiography or CENTRA) for the upper and lower leg arteries to reduce the 
risk of venous enhancement (12). In all three stations a dedicated, $129 \mathrm{~cm}$ long, 12 element phased array peripheral vascular reception coil was used (Philps Medical Systems, Best, The Netherlands). This coil has a flexible twowpiece design, with one piece placed posterior to the patient and one piece placed anterior to the patient. During acquisition of each separate station, the corresponding 2 anterior and 2 posterior elements of the coil are selected via the software and used simultaneously for signal reception.

Each patient received a standard dose of $35 \mathrm{~mL}$ of gadolinium DTPA (Magnevist, Schering, Berlin, Germany) administered at a rate of $1.8 \mathrm{~mL} / \mathrm{s}$ for the first $15 \mathrm{~mL}$, and $0.6 \mathrm{~mL} / \mathrm{s}$ for the remaining $20 \mathrm{~mL}$ (total contrast injection duration: $42 \mathrm{~s}$ ), followed by a saline flush of $20 \mathrm{~mL}$ at $0.6 \mathrm{~mL} / \mathrm{s}$. All injections were done with a remote controlled MR compatible injection system (Medrad Spectris, Indianola, PA, USA). Table occupancy for this protocol was approximately 20 minutes. Reconstruction and postprocessing required on average an additional 20 minutes.

\section{CE-MRA Protocol Using SENSE}

The imaging protocol using SENSE was identical to the non-SENSE protocol in terms of patient positioning, acquisition of TOF scout scans and use of the dedicated, three-station peripheral vascular coil. However, in each station a reference scan was made to obtain sensitivity profiles for unfolding pixels in the undersampled direction. These gradient echo reference scans were acquired prior to the non contrastenhanced mask scans with the following imaging parameters: TR (ms) / TE (ms) / flip angle (degrees) / acquisition time (s): 8.0/0.79/7/60. Imaging parameters for the SENSE CE-MRA acquisitions are also listed in table 2.

In the present implementation a maximum SENSE factor of 2 (with undersampling in the left-to-right phase encoding direction) was possible, i.e. the acquisition time could be halved. However, a requirement for the use of SENSE is that the field-of-view in the SENSE phase-encode direction covers the entire object in that direction; if this is not the case, potentially disturbing aliasing artifacts are created in the central part of the images. Based on this boundary condition we chose not to use parallel imaging in the pelvic arteries. This was because we would have to increase the value for the FOV in the left-to-right direction with $30-40 \%$ in order to avoid aliasing and therefore the acquisition duration would not be substantially shorter for most patients. Instead, Constant LEvel AppeaRance (CLEAR) was used for the aortoiliac station to improve coil sensitivity and to suppress noilse in the images. CLEAR corresponds to SENSE reduction factor one, and does not lead to a reduction in acquisition time. For the upper and lower leg stations k-space was undersampled twofold (SENSE factor 2) in the left-to-right phase encoding direction. The resultant reduction in scan time was partially applied to improve the truly measured spatial resolution from 4.7 to $3.9 \mathrm{~mm} 3$ in the upper legs (table 2). In the lower legs, the time savings were used with the objective to decrease venous enthancement and to increase the number of partitions to include the pedal arch. The FOV was not increased for the upper and lower legs in case it did not cover the entire patient in the SENSE phase encoding direction (left-right), because aliasing artefacts 
in the central part of the image are almost never disturbing as there is generally only air between the legs and no arteries.

To offset some of the expected loss in signal-to-noise ratio, the injection protocol was changed. The rate at which gadolinium was administered was empirically increased from 1.8 to $2.0 \mathrm{~mL} / \mathrm{s}$ for the first $15 \mathrm{~mL}$, and from 0.6 to 0.9 $\mathrm{mL} / \mathrm{s}$ for the remaining $20 \mathrm{~mL}$ (total contrast injection duration: $30 \mathrm{~s}$ ), followed by a saline flush of $20 \mathrm{~mL}$ at $0.6 \mathrm{~mL} / \mathrm{s}$. Table occupancy was slightly longer $(3-4 \mathrm{~min})$ compared to the conventional imaging protocol, because of the requirement to perform reference scans. Reconstruction and postprocessing also took approximately 20 minutes.

Table 2 Imaging parameters for the SENSE and non-SENSE 3D CE-MRA protocols

\begin{tabular}{|c|c|c|c|c|c|c|}
\hline & $\begin{array}{l}\text { Aortoiliac } \\
\text { non-SENSE }\end{array}$ & CLEAR & $\begin{array}{l}\text { Upper legs } \\
\text { non-SENSE }\end{array}$ & SENSE & $\begin{array}{l}\text { Lower legls } \\
\text { non-SENSE }\end{array}$ & SENSE \\
\hline TR (msec) & 4.7 & 4.8 & 4.7 & 4.9 & 4.0 & 4.0 \\
\hline TE (msec) & 1.4 & 1.4 & 1,4 & 1.4 & 1.4 & 1.4 \\
\hline FA (degrees) & 40 & 40 & 40 & 40 & 30 & 30 \\
\hline FOV-freq $(\mathrm{mm})$ & 470 & 470 & 470 & 470 & 470 & 470 \\
\hline FOV-phase (mm) & 259 & 258 & 259 & 305 & 259 & 282 \\
\hline Matrix (freq) & 368 & 384 & 352 & 384 & 480 & 480 \\
\hline Matrix (phase) & 155 & 156 & 155 & 200 & 264 & 288 \\
\hline Slice thickness & 2.8 & 2.6 & 2.1 & 2.1 & 1.5 & 1.5 \\
\hline Voxel size (mm3) & $6.0^{*}$ & $5.3^{*}$ & $4.7^{*}$ & $3.9^{*}$ & $1.4^{*}$ & $1.4^{*}$ \\
\hline Number of slices & $30-35$ & $26-29$ & $20-25$ & $24-30$ & $65-70$ & 80 \\
\hline SENSE reduction factor & none & none & none & 2 & none & 2 \\
\hline Acquisition duration (sec) & $22-25 t$ & $19-22 \dagger$ & $15-18 \dagger$ & $12-15 \dagger$ & $69-74 \dagger$ & $45 \dagger$ \\
\hline Partial echo $(67.7 \%)$ & yes & yes & yes & yes & yes & yes \\
\hline Half scan $\neq$ & no & no & no & no & no & no \\
\hline Phase encoding order & linear & linear & CENTRA & CENTRA & CENTRA & CENTRA \\
\hline
\end{tabular}

CLEAR = Constant LEvel AppeaRance; FA = flip angle; FOV = field-of-view; freq = frequency encoding direction; phase = phase encoding direction; * Actually measured voxel sizes, these voxels were later reconstructed to half their size by zero interpolation in the slice direction; $t$ the exact duration of each station varied per patient with the number of partitions used in each station. $\ddagger$ Half scan $=$ half Fourier imaging (or half number of excitations) in the phase-encoding direction $(75 \% \mathrm{~K}$ space filling).

\section{Intra-Arterial Digital Subtraction Angiography}

All IA-DSA examinations were supervised by experienced interventional radiologists who were unaware of CE-MRA findings. Patients were imaged using either Philips Integris V5000 (Philips Medical Systems, Best, The Netherlands) or Siemens Polystar (Siemens Medical Systems, Erlangen, Germany) digital angiography equipment. In 31 patients aortic flush arteriograms were obtained using 4 French universal flush catheters (Cordis, Miami, FL) placed in the infrarenal aorta. A total of 100-120 $\mathrm{mL}$ contrast medium (lohexol, Omnipaque, $300 \mathrm{mg} / / \mathrm{mL}$, Nycomed 
Amersham. Eindhoven. The Netherlands) was injected at flow rates ranging from 4 to $12 \mathrm{~mL} / \mathrm{s}$. In 7 patients selective arteriograms were obtained using an antegrade puncture technique where the catheter was placed in the common femoral artery or distal thereof with a 4 French sheath (Cordis "Miami, FL). The total amount of contrast medium used in these cases varied between 60 to $80 \mathrm{~mL}$. The number and orientation of views obtained with both techniques was at the discretion of the supervising radiologist.

\section{Image Analysïs}

For both MRA and IA-DSA anallysis, the arterial tree was divided into 29 segments: infrarenal abdominal aorta, both left and right common iliac, external iliac, common femoral, superficial femoral (proximal and distal), popliteal (above and below knee), tibeoperoneal trunk, anterior tibial (proximal and distal), posterior tibial (proximal and distal), and peroneal (proximal and distal) arteries.

For every vessel segment that was considered evaluable, signal-to-noise ratio (SNR) and contrast-to-noise ratio (CNR) were determined in all 38 patients. SNR was determined on the original contrast-enhanced partitions by drawing a region of interest (ROI) within the vessel segment and dividing the mean signal value by the standard deviation of noise measured outside the patient. CNR was determined by subtracting the signal intensity measured in a ROI immediately adjacent to the vessel segment from intravascular values and dividing by the standard deviation of noise measured outside the patient. No corrections were made to adjust for different voxel sizes.

To compare possible differences in venous enhancement between the nonSENSE and SENSE groups the frequency as well as the severity of venous enhancement was assessed for each station. Severity was classified using the following categories: superficial and/or deep venous enhancement rendering one or more vessel segments uninterpretable; superficial and deep venous enhancement present but not disturbing interpretation; superficial venous enhancement present but not disturbing interpretation, and no venous enhancement.

In addition, a subjective interpretability score (SIS) was given for each station, based on review of individual partitions of the subtracted contrast-enhanced $3 \mathrm{D}$ volumes and whole volume MIP images. SIS ranged from $0=$ not sufficient for further treatment planning; 1 = suboptimal image interpretability but sufficient for further treatment planning; and 2 = good interpretability and sufficient for further treatment planning.

Finally, we investigated potential differences in diagnostic accuracy between the non-SENSE and SENSE CE-MRA protocols. For this purpose one experienced observer, blinded for clinical information and IA-DSA results, assessed the presence or absence of hemodynamically significant stenoses (defined as $250 \%$ linear diameter reduction) and occlusions on MRA. Another observer, unaware of the CEMRA and clinical findings, assessed the IA-DSA images in similar fashion. For the pedal arch both observers only assessed if this vessel was patent or not. Based on this, sensitivity and specificity of both non-SENSE and SENSE techniques were calculated and compared on a station-by-station basis. 


\section{Statistical Analysis}

SNR, CNR, SIS and VES were compared for both groups using the Mann-Whitney U test for two independent samples. The Mann-Whitney $U$ test was used because it was assumed that there was no normal distribution. Comparisons were made on a station-by-station basis. The Chi-square test was used to compare if there was a difference in sensitivity and specificity between the SENSE and the non-SENSE group. A P-value less than 0.05 was taken to indicate a statistically significant difference. All statistical analyses were performed using SPSS (Version 10.0, SPSS Inc. Chicago, $\mathrm{L}$ ).
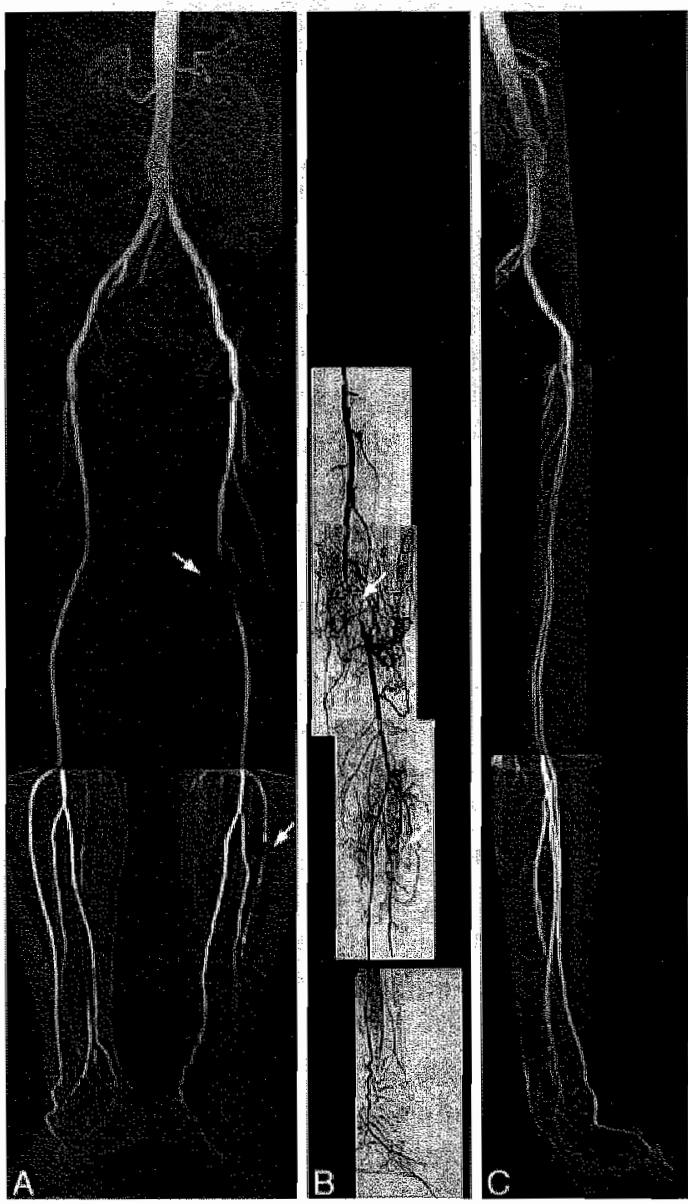

Figure 1

Non-SENSE peripheral CE-MRA and corresponding IA-DSA in a 68 year old male with moderate complaints of intermittent claudication in the left leg (Fontaine II b peripheral arterial disease). The left panel a: shows a whole volume coronal maximum intensity projection (MIP) of the acquired dataset. The middle panel $b$ : shows the corresponding coronal antegrade $A$ A-DSA of the left upper and lower leg the distal lower leg and foot are detached because this is a lateral projection). The right panel $c$ : shows a lateral view of the same CEMRA dataset. Both the CE-MRA and the IA-DSA show an occlusion in the left superficial femoral artery (upper arrows), and an occlusion in the left anterior tibial artery (llower arrows). 


\section{Results}

In 35 patients MRA examinations from the aortoiliac arteries down to and including the lower legs were obtained. In the remaining three patients (all in the non-SENSE group) the lower legs were not depicted. In two patients, this was because the referring clinician also requested information about the thoracic aorta, thereby excluding the lower leg station. In one patient, the lower leg station scan failed due to incorrect timing of table movement. Therefore, the lower legs were depicted in only 16 patients of the non-SENSE group. In figure 1, a representative example is shown of a patient imaged using the non-SENSE protocol. In figures 2 and 3 examples are shown of patients imaged with the parallel imaging protocoll. Total imaging time, from setup to diagnosis toak 40 to 45 minutes for both SENSE and non-SENSE protocols.
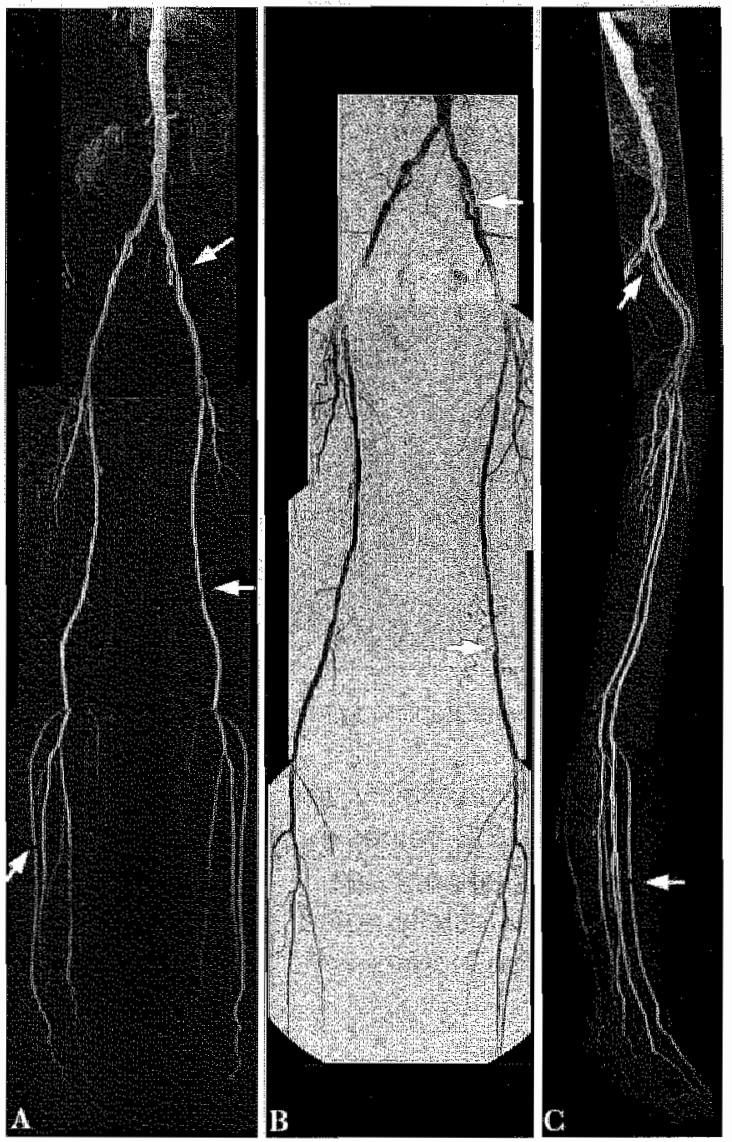

Figuire 2

Peripheral CE-MRA using SENSE and IA-DSA in a 73 year old femble with bilateral moderate complaints of intermittent claudication. The left panel a: shows a coronal MIP. In the middle panel $b$ : the corresponding IA-DSA can be seen. The right panel C. shows a sagittal (laterall) view of the same CE-MRA dataset. There is a high grade stenosis in the left external illac antery (upper arrows). In addition, there is a high grade stenosis in the left femoral superficial artery on both IA-DSA and CE-MRA (middle arrows). On the CE-MRA images a stenosis in the right anterior tilbial antery can also be seen (lower arrows) which was not depicted on IADSA because of limited anatomical coverage.

In table 3 , the results of the SNR and CNR measurements are listed. For the aortoiliac station, SNR and CNR increased significantly by $62 \%$ and $45 \%$ (both P< $.0011)$ when CLEAR was used. For the upper leg station, SNR and CNR also increased significantly by $88 \%$ and $90 \%$ both $P<.001$ ) when SENSE was used. There was no significant difference in SNR and in CNR between the SENSE and 
control groups with regards to the lower legs. Both SNR and CNR decreased slightly $(-3 \%)$ in the SENSE group, which was not significant $(P=.3)$.

Table 3 Mean Signal to noise ratio and Contrast to noise ratio measured in 3 stations.

\begin{tabular}{lllllll}
\hline $\begin{array}{l}\text { Mean } \\
( \pm S D)\end{array}$ & $\begin{array}{l}\text { Aortoiliac } \\
\text { non-SENSE }\end{array}$ & CLEAR & $\begin{array}{l}\text { Upperlegs } \\
\text { nan-SENSE }\end{array}$ & SENSE & $\begin{array}{l}\text { Lower legs } \\
\text { non-SENSE }\end{array}$ & SENSE \\
\hline SNR & $34( \pm 7)$ & $55( \pm 16) \dagger$ & $26( \pm 12)$ & $49( \pm 21) \dagger$ & $34( \pm 5)$ & $33( \pm 7)$ \\
CNR & $31( \pm 8)$ & $45( \pm 13) \dagger$ & $22( \pm 11)$ & $42( \pm 19) \dagger$ & $30( \pm 5)$ & $29( \pm 7)$ \\
\hline
\end{tabular}

$\mathrm{SD}=$ standard deviation; $\uparrow$ significantly higher compared to non-SENSE imaging protocol $(\mathrm{P} \leq .001)$.

There was a trend towards less venous contamination with the use of parallel imaging, but this was not statistically significant (table 4). We observed a $14 \%$ reduction of disturbing venous enhancement in the lower leg station in the SENSE group $(2 / 19 ; 11 \%)$ compared to the control group $(4 / 16 ; 25 \%)$. Both the non-SENSE and the SENSE group did not show any disturbing venous enhancement in the aortoiliac or upper leg stations.

Table 4 Distribution of patients over different degrees of venous enhancement as measured in the lower leg station

\begin{tabular}{llll}
\hline & Frequency & & \\
Number of Patients & non-SENSE & SENSE & P-value \\
\hline No venous & $5 / 16^{*}(31 \%)$ & $10 / 19(53 \%)$ & 1.0 \\
Superficial & $5 / 16^{*}(31 \%)$ & $3 / 19(16 \%)$ & 0.8 \\
Deep, interpretable & $2 / 16^{*}(13 \%)$ & $4 / 19(21 \%)$ & 0.2 \\
Deep, not interpretable & $4 / 16^{*}(25 \%)$ & $2 / 19(11 \%)$ & 0.4 \\
\hline
\end{tabular}

*In only 16 patients of the non-SENSE group the lower legs were depicted (see text).

The results with regards to subjective interpretability are shown in table 5 . Images in the SENSE group had somewhat better SIS values in aortoiliac and upper leg stations, but these differences were not significant (all $P>.05$ ). However, improvements in subjective interpretability were especially noted in the lower legs of the patients that underwent the SENSE protocol (an increase of $21 \%$ ), however this difference was also not significant $(P=.4)$. 

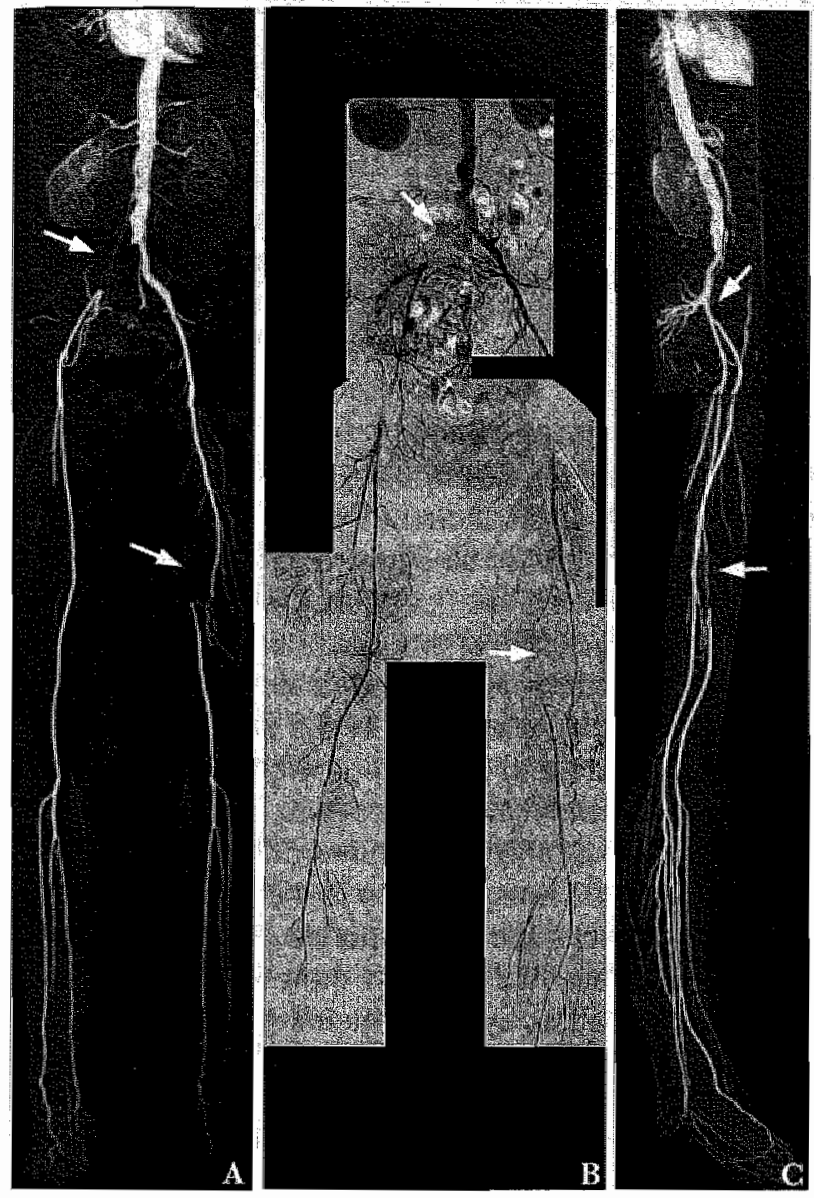

Figure 3

CE-MRA Using SENSE and corresponding IA-DSA in a 69 year old female with moderate complaints in the figint leg. In the left panel a: a coronal MIP image of the acquired dataset is shown. In the middle panel b: the corresponding 1A-DSA and the right panel $c$. the sagittal (lateral) view of the same CE-MRA dataset can be seen. Both IA-DSA and CEMRA show an occtusion in the right common lliac artery (upper arrows) and an occlusion in the left superficial femoral artery (lower arrows) of similar length and degree of severity.

Note better opacification of distal runoff arteries on CEMRA.

Table $5 \quad$ Comparison of Subjective Interpretability Score

\begin{tabular}{llll}
\hline Mean ( \pm SD) & non-SENSE & SENSE & P-value \\
\hline Aortoiliac & $1.8( \pm 0.5)$ & $2.0( \pm 0)$ & 0.4 \\
Upper legs & $1.9( \pm 0.2)$ & $2.0( \pm 0.2)$ & 1.0 \\
Lower legs & $1.4( \pm 0.8)$ & $1.7( \pm 0.7)$ & 0.4 \\
\hline
\end{tabular}

The results with regards to diagnostic accuracy of SENSE and non-SENSE imaging protocols in comparison to IA-DSA are shown in table 6. Sensitivity and specificity were not significantly different for any of the three vascular regions between the patients that underwent the SENSE protocol and the patients that were imaged using our previous, non-SENSE protocol, although the sensitivity in the lower leg station was improved when SENSE was used. 
Table 6 Diagnostic accuracy of SENSE and non-SENSE imaging protocols

\begin{tabular}{lllllll}
\hline & $\begin{array}{l}\text { Sensitivity } \\
\text { Non-SENSE }\end{array}$ & SENSE & P-walue Non-SENSE & SENSE & P-value \\
\hline Aontollac & $91 \%(21 / 23)$ & $85 \%(11 / 13)$ & 1.0 & $97 \%(91 / 94)$ & $97 \%(65 / 68)$ & 1.0 \\
Upperlegs & $68 \%(20 / 29)$ & $74 \%(14 / 19)$ & 0.7 & $93 \%(94 / 101)$ & $96 \%(90 / 94)$ & 0.5 \\
Lowerlegs & $78 \%(25 / 32)$ & $87 \%(13 / 15)$ & 0.7 & $95 \%(136 / 140)$ & $95 \%(146 / 154)$ & 0.4 \\
\hline
\end{tabular}

\section{Discussion}

The present study shows that the implementation of parallel imaging for peripheral CE-MRA is feasible. Parallel imaging allows for additional flexibility in the choice of MR imaging parameters and optimization in terms of objective and subjective image quality. When parallel imaging is combined with the use of a 3-station peripheral vascular coil it is possible to routinely depict the entire peripheral vascular tree including the pedal arch without much disturbing venous enhancement. This is of great importance for distal arterial bypass graft planning. In the current study we employed a SENSE factor of 2 for the upper and lower legs which resulted in a modest reduction in venous enhancement and small improvements in subjective interpretability and diagnostic accuracy. On the other hand, SNR and CNR improved significantly, which was manifested as improved image quality in the figures as well.

The present study corroborates the results recently reported by Maki et al who used parallel imaging for total runoff peripheral CE-MRA in the distal station (10). They reported improved calf artery visualization by implementing SENSE in one station for speeding up the acquisition time. In contrast to the current study the pedal arch was, however, not routinely depicted.

The cause of the improved SNR and CNR is not completely clear. For the aortoiliac station this might be explained by faster contrast agent injection and the use of CLEAR (thereby inducing noise suppression). In image reconstruction from sensitivity-encoded data, noise enhancement occurs in regions where the geometric relations of coil sensitivities are not optimal. This effect is described by the local geometrical noise factor $\mathrm{g}$. The latter is a mathematical function of the coil sensitivities and of the SENSE reduction factor, with geometric factors usually growing as the reduction factor increases. Thus, when using parallel imaging one expects a drop in SNR and CNR proportional to g (13). Moreover, the geometry factor, and consequently SNR and CNR, are spatially variable. Ideally, this needs to be incorporated for the most accurate SNR and CNR calculations. For the upper and lower leg stations, however, values for SNR and CNR are artificially lowered when SENSE is used because measured noise contains real noise but also signal variations due to coil setup and aliasing artifacts (because of incomplete coverage of the upper and lower legs in the SENSE phase-encode direction). Still, SNR and CNR increased with the use of SENSE, which is most likely explained by the increase in contrast medium injection speed, and the increased number of partitions in the upper and lower leg stations. 
Theoretically, the use of SENSE offers synergistic benefits for reduction of venous enhancement in the lower legs when used in combination with optimized centric phase encoding. This is not only because the data points in the center of Kspace (which encode contrast information) for the lower legs are collected earlier, but also because the speed by which K-space is traversed from the center outwards increases by the acceleration factor used. Therefore, one of the most important improvements in the current: study was the reduction in scan duration from $132 \mathrm{~s}$ in the control group to $80 \mathrm{~s}(-39 \%)$ when parallel imaging was used.

The time that became available because of the use of SENSE in two stations was invested in a combination of reducing temporal resolution (to avoid venous enhancement'), increasing spatial resolution, and increasing the number of slices for more complete anatomical coverage. Even though scan time was reduced, we were able to further decrease voxel sizes. Despite the reduction in acquisition duration by using SENSE, we were still not able to completely prevent venous enhancement in the lower legs. This means that, in some cases, our acquisition duration is still too long to avoid venous enhancement. Venous enhancement occurs because the lower leg acquisition usually starts past peak arterial contrast medium concentration and during increasing venous concentration. It is known that intravenously injected contrast material can travel down the peripheral arteries very rapidly (in some patients at approximately 6 seconds per station (14)). Therefore a $39 \%$ reduction in total acquisition time obtained with the use of parallel imaging technique may not be sufficient for elimination of venous enhancement in all patients. To completely overcome venous enhancement higher parallel imaging-factors are needed to further reduce total acquisition duration. Alternative methods for preventing venous enhancement are the use of multiple injections with 2D (15) or 3D (16) Cartesian acquisitions of the lower leg first or the use of time resolved imaging of contrast kinetics (TRICKS) (17). With the latter imaging technique high-resolution 3D images of the lower legs every few seconds by updating the central part of k-space more often than the periphery. The advantage of using these techniques is that almost always at least one image with peak arterial and very little venous filling will be depicted. Drawbacks are a lower signal to noise ratio due to the lower amount of contrast medium that is given to image the lower legs only and the increased imaging and post-processing time. The additional advantage of using a $2 \mathrm{D}$ time resolved technique for the lower legs is that a subsequent $3 \mathrm{D}$ high resolution examination of the entire peripheral vascular tree can be adapted to variations between patients in contrast travel time to the calf (18). Encouraging results with regard to reducing venous enhancement have also been obtained by using infrasystolic midfemoral or lower leg cuff-compression (19,20).

In the current study no significant differences were found with regard to subjective image quality although image quality was indeed higher in the lower leg station. Given the minimal changes in imaging parameters (except for reduction in acquisition duration) this is perhaps not surprising. In contrast, the maintenance of subjective image quality is encouraging because it shows that parallel imaging is easy to combine with different imaging protocols and k-space trajectories, without major artifacts. As described earlier in the discussion on venous enhancement, 
higher reduction factors in all three stations are probably needed to take optimal advantage of parallel imaging for peripheral CE-MRA.

With regards to diagnostic accuracy no significant differences were observed. When SENSE was used sensitivity decreased somewhat for the aortoiliac station, but increased for the upper and lower legs. This might be explained by the reduction of venous enhancement and depiction of pedal arch when SENSE was used. We must comment, however, that the sensitivity we found in the control group was somewhat lower when compared to a recent meta-analysis of peripheral CE-MRA studies, that showed sensitivities between $81-100 \%$ in the aortoiliac station, $98-100$ $\%$ in the upper leg station and $92-100 \%$ for the lower legs (1). The lower values of sensitivity might be explained by the case mix and/or the relatively small patient group included in this feasibility study.

The current study has limitations, the most important of which is that the two imaging protocols were not compared in the same patient group. However, we believe that the impact of this may be relatively small since both the control and SENSE protocol patient populations suffered from similar degrees of peripheral arterial occlusive disease. A second limitation is that resolution (matrix slice thickness) and coverage (number of slices) parameters as well as acquisition duration and injection rate were changed all at once. If the time gain associated with the use of parallel imaging had been exclusively used for reducing scan time, a lower rate of venous enhancement may have been observed. Because multiple parameters were changed we did not expect to detect largle differences in the outcome parameters between the control group and the group where parallel imaging was used. However, the aim of the current study was to prove the feasibility of using parallel imaging in two stations for peripheral CE-MRA, and to explore possible problems in the implementation thereof. An important insight of the current study is that only SENSE reduction factors of greater than 2 are likely to confer significant increases in image quality and diagnostic accuracy. Another important limitation is that we did not take into account the variations in noise as a function of space. This has influenced the values we found for SNR and CNR. However, our primary aim was to investigate if there was no major loss of image quality when using parallel imaging.

In conclusion, preliminary results show that parallel imaging with SENSE in combination with a three station surface coil is useful for further optimization of peripheral CE-MRA in terms of coverage of the pedal arch and improved vessel to background contrast. These improvements are clinically important because they may allow peripheral MR angiography to be used with more confidence as a planning tool for distal vascular interventions. Higher parallel imaging reduction factors are needed to optimize these benefits. 


\section{References}

1. Nelemans PJ, Leiner $T$, de Vet $H C$, van Engelshoven $\mathrm{MM}$. Peripheral arterial disease. metaanalysis of the diagnostic performance of MR angiography. Radiology 2000, 217(1):105-114.

2. Carpenter JP, Baum RA, Holland GA, Barker CF. Peripheral vascular surgery with magnetic resonance anglography as the sole preoperative imaging modality: J Vasc Surg 1994; 20(6):861-869; discussion 869-871.

3. Visser $K_{n}$ Hunink MG. Peripheral arterial disease: gadolinium-enhanced MR angiography versus color-guided duplex US--a meta-analysis. Radiology 2000; 216 (1):67-77.

4. Ho KY, Leiner T, de Haan MW, van Engelshoven JM. Peripheral MR angiography. Eur Radiol $1999 ; 9(9): 1765-1774$.

5. Hood MN, Ho VB, Foo TK, Marcos HB, Hess SL "Choyke PL. High-resolution gadoliniumenhanced 3D MRA of the infrapopliteal arteries. Lessons for improving bolus-chase peripheral MRA Magn Reson Imaging 2002; 20(7):543-549.

6. Carpenter JP, Golden MA, Barker CF, Holland GA, Baum RA. The fate of bypass grafts to angiographically occult runoff wessels detected by magnetic resonance angiography. $₫$ Vasc Surg 1996; 23(3):483-489.

7. Huber TS, Back MR, Ballinger RJ, et al. Utility of magnetic resonance arteriography for distal lower extremity revascularization. J Vasc Surg 1997; 26(3);415-423; discussion 423-414.

8. Pruessmann KP, Weiger M, Scheidegger MB, Boesiger $P$, SENSE: sensitivity encoding for fast MRl. Magn Reson Med 1999; 42(5):952-962.

9. Kurihara $Y$, Yakushiji $Y K$, Tani I, Nakajima $Y$, Van Cauteren M. Coil sensitivity encoding in MR imaging: advantages and disadvantages in clinical practice. AJR Am J Roentgenal $2002 ; 178 ;(5): 1087-1091$.

10. Maki JH, Wilson GJ, Eubank WB, Hoogeveen RM. Utilizing SENSE to achieve lower station sub-millimeter isotropic resolution and minimal venous enhancement in peripheral MR angiography. J Magn Reson Imaging 2002;15(4):484-491.

11. Maki $\mathrm{JH}$, Prince MR, Chenevert TC. Optimizing three-dimensional gadiolinium-enhanced magnetic resonance angiography. Original investigation. Invest Radiol 1998; 33(9):528-537.

12. Willinek WA, Gieseke $J_{\|}$Conrad $R$, et al. Randomly segmented central k-space ordering in highspatial-resolution contrast-enhanced MR angiography of the supraaortic arteries: initial experience. Radiology 2002; 225(2):583-588.

13. Weiger $M$, Pruessmanm KP, Kassner $A$, et al. Contrast-enthanced 3D MRA using SEINSE. J Magn Reson Imaging 2000; 12(5):671-677.

14. Prince $M R_{3}$, Chabra SG, Watts $R$, et al. Contrast material travel times in patients undergoing peripheral MR angiography. Radiology 2002; 224(1):55-61.

15. Wang $Y$, Winchester PA, Khilnani NM, et al. Contrast-enhanced peripheral MR angiography from the abdominal aorta to the pedal arteries: combined dynamic two-dimensional and boluschase three-dimensional acquisitions. Invest Radiol 2001; 36(3):170-177.

16. Morasch MD, Collins J. Pereles FS, et al. Lower extremity stepping-table magnetic resonance angiography with multilevel contrast timing and segmented contrast infusion. J Vasc Surg 2003; 37(1):62-71.

17. Swan JS, Carroll $T J$, Kennell TW, et all. Time-resolved three-dimensional contrast-enhanced MR angiography of the peripheral vessels. Radiology $2002 ; 225$ (1):43-52. 
18. Wang $Y$, Chen $\mathrm{CZ}$, Chabra $\mathrm{SG}$, et al. Bolus arterial-venous transit in the lower extremity and venous contamination in bolus chase three-dimensional magnetic resonance angiography. Invest Radiol 2002; 37(8):458-463.

19. Bilecen $D$, Schulte $A C$, Bongartz $G_{*}$ Heidecker $H G$, Aschwanden $M_{*}$ Jager $K A$. infragenual cuffcompression reduces venous contamination in contrast-enhanced MR angiography of the calf. J Magn Reson lmaging 2004, 20(2):347-351.

20. Herborn CU, Ajaj W, Goyen M, Massing S, Fuehm SG, Debatin JF. Peripheral Vasculature: Whole-Body MR Angiography with Midfemoral Venous Compression-Initial Experience. Radiology 2004: 230(3):872-878. 


\section{Chapter 3}

\section{Comparison of generic and disease-specific questionnaires for the assessment of quality-of-life in patients with peripheral arterial disease}

Journal of Vascular Surgery 2005: 41:2: 261-268

Marianne de Vries, Rody Ouwendijk, Alphons G. Kessels, Michiel W. de Haan, Karin Flobbe, M.G. Myriam Hunink, Jos M.A.van Engelshoven, Patricia J. Nelemans, 


\section{Abstract}

\section{Purpose}

The purpose of the present study was to compare the ability of generic and diseasespecific questionnaires to assess quality of life (QoL) at baseline and to detect change in QoL after treatment in patients with peripheral arterial disease (PAD).

\section{Materials and Methods}

In the prospective multicenter trial, 514 patients with PAD who needed imaging workup and had an ankle brachial pressure index (ABPI) $<0.90$ were included. Patients with severe co-morbidity were excluded, leaving a study population of 450 patients. Patients completed two generic questionnaires (SF-36 and Euroqol-5D) and one disease-specific questionnaire (VascuQol) at baseline and after 6 months of follow-up. Rutherford classification, treadmill walking distance, were determined at baseline and after 6 months of follow-up and were considered indicators of disease severity. Each of the three questionnaires was evaluated for its ability to discriminate between severe and mild disease at baseline, and to discriminate between a large and small change in disease severity after follow-up, using receiver operating characteristic (ROC) curves and areas under the curves (AUCs). The underlying assumption was that disease severity is a major determinant of QoL. This implies that the validity of a QoL questionnaire is reflected by its ability to discriminate between mildly and severely diseased patients.

\section{Results}

At baseline 443 patients and after follow-up 386 patients completed questionnaires. At baseline no significant (P>.05) differences were observed between AUCs for the total scores of the three questionnaires, indicating that all three questionnaires assessed the disease severity equally well. After follow-up the AUCs for the VascuQol were significantly higher than the AUCs for the SF-36 and Euroqol-5D, with respect to detection of improvement in Rutherford classification $(\mathcal{A}<.05)$, indicating that change in disease severity after follow-up was best detected by the VascuQol.

\section{Conclusion}

The VascuQol is the preferred questionnaire as outcome measure for QoL in future trials and clinical follow-up of patients with PAD. 


\section{Introduction}

Peripheral arterial disease (PAD) is a chronic condition with a high morbidity which is reflected in an impaired quality-of-life $(\mathrm{QoL})^{(1-3)}$. The major treatment goal for $\mathrm{PAD}$ is to improve functional status of the patient and to relieve disability. Treatment of PAD is usually evaluated in terms of clinical parameters such as Rutherford classification, ankle brachial pressure index (ABPI) and walking distance. A major drawback of these clinical parameters is that they poorly reflect the perspective of the patient after treatment ${ }^{(4-6)}$. As a result several studies propose measurement of $\mathrm{QoL}$ as a primary endpoint in evaluating treatment effects $(7-11)$. These studies have, however, used a wide variety of different questionnaires to evaluate $\mathrm{QoL}$, because it is still unclear which questionnaire best serves this purpose.

One approach to measure $\mathrm{QoL}$ is the use of generic health questionnaires such as the Short Form 36 (SF-36) or the Euroqol-5D, which measure physical, social and emotional dimensions of health. The advantage of generic questionnaires is that they can be used for evaluating QoL for all kind of diseases, and for calculating utility values in cost-effectiveness analysis. However, these generic questionnaires are considered to be less sensitive to detect small but clinically important differences in treatment effects, because they do not focus on specific effects of disease ${ }^{(12)}$.

Another approach for assessing QoL is the use of disease-specific questionnaires in patients with PAD, such as the VascuQoll (9). The VascuQol measures more specific elements of peripheral arterial disease, and thus theoretically, is more responsive to measure more subtle effects after treatment ${ }^{(13)}$.

To date, little is known about the comparative validity between generic and disease-specific questionnaires in patients with PAD. Comparative studies are needed to determine which questionnaire has the best ability to assess QoL at baseline and to capture change in QoL after treatment. A comparative study of Morgan et al. used the SF-36 mainly for validation of the VascuQol, instead of determining which questionnaire should be preferred in the assessment of QoL in patients with PAD ${ }^{(13)}$

The present study compared the ability of generic with disease-specific questionnaires to assess QoL at baseline and to detect change in QoL after treatment in patients with PAD.

\section{Material and Methods}

\section{Patients and Questionnaires}

The present study was part of a large prospective Dutch multicenter study in which patients with PAD were randomized between MR angiography and the locally employed imaging protocol, either duplex or CT angiography, to compare imaging work-up strategies. Four centres in the Netherlands participated in the study.

Between December 2001 and September 2003 all patients with PAD, who needed imaging workup to evaluate the feasibility and choice of revascularisation 
procedure, were included. The study population consisted of 514 patients with intermittent claudication or critical ischaemia and an ankle brachial pressure index (ABPI) $<0.90$. Clinical utility, patient outcome, and costs between the different imaging work-up strategies were compared. For this purpose clinical parameters and QoL were collected at baseline and six months after imaging work-up (Figure 1).

Figure 1 Flow diagram of the study and data collection

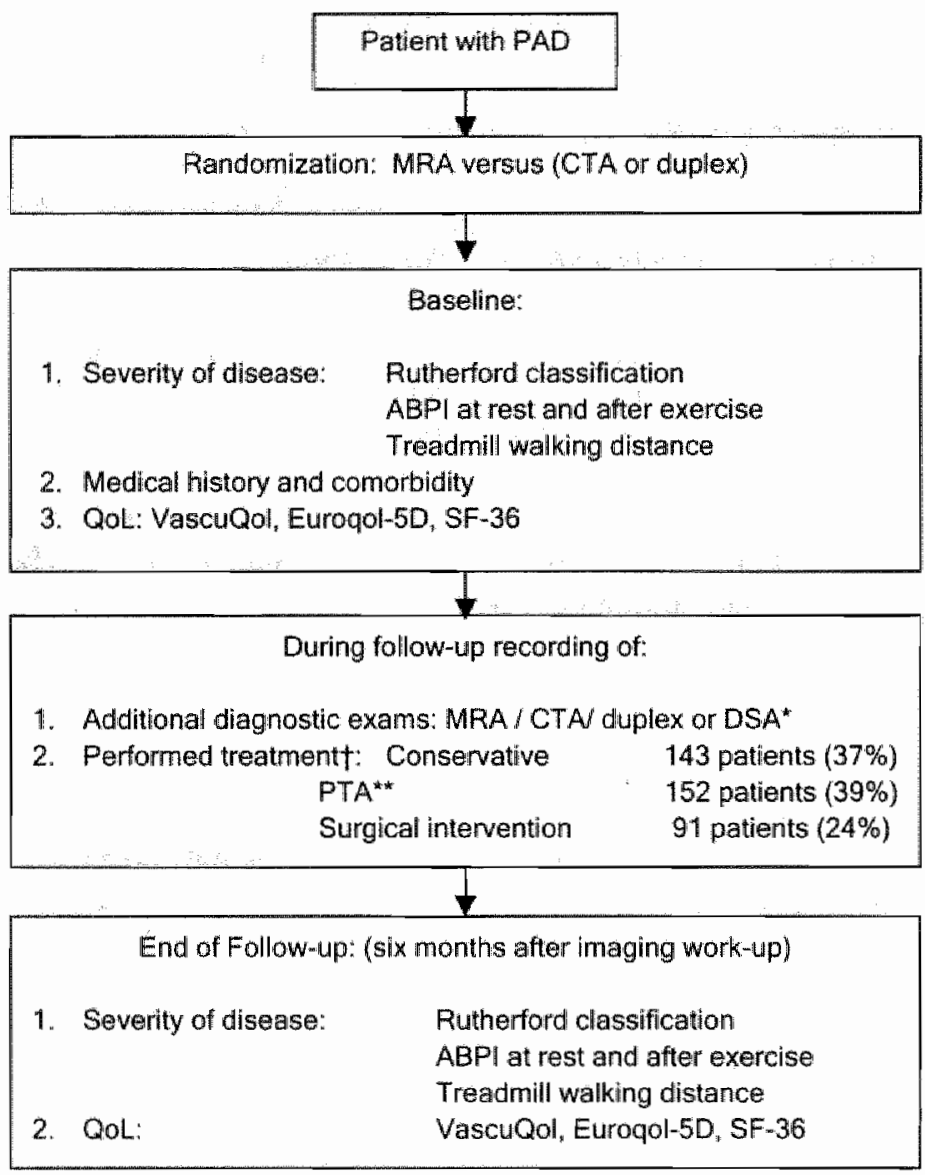

$\mathrm{PAD}=$ peripheral arterial disease; $M R A=$ magnetic resonance angiography; $C T A=$ computed tornographic angiography; $A B P I=$ ankle brachial pressure index; $D S A=$ digital subtraction angiography. ${ }^{*} \mathrm{PTA}=$ percutaneous translurninal angioplasty. After follow-up 386 patients returned their questionnaire. The percentages are calculated using these 3.86 patients.

Excluded because they could not participate in the randomized trial were patients who needed an imaging workup within 3 days, patients with contraindications for MR angiography and CT angiography, and patients who already had had an imaging workup indicating that revascularisation was needed. The present study also excluded patients with severe co-morbidity in order to avoid 
influence of co-morbidities affecting QoL on the comparison of disease-specific and generic questionnaires. Of the 514 patients who were included in the study, 64 patients with severe co-morbid diseases were excluded, leaving a study population of 450 patients without severe co-morbidity.

All patients signed written informed consent prior to the randomization. All patients completed two generic questionnaires (SF-36 and Euroqol-5D) and a disease-specific questionnaire (VascuQol). Patients returned their questionnaires by mail at baseline and six months after imaging work-up.

\section{Short Form 36 (SF-36)}

The SF-36 is a well established generic questionnaire and has often been used for patients with various diseases (14). The SF-36 comprises eight different health dimensions. In the present study we used four of these eight health dimensions: physical functioning (10 items), role physical functioning (4 items), bodily pain (2 items) and general health (5 items). We selected these four dimensions, because these have shown to be the most responsive for measuring changes in QoL after treatment of patients with PAD ${ }^{(8,13,15)}$. Patients' responses were converted to a scale ranging from 0 (worst possible score) to 100 (best possible score).

\section{Euraqol-5D}

The other employed generic questionnaire was the Euroqol-5D. It contains five questions regarding mobility, self-care, usual activities, pain and anxiety / depression with each three levels of severity corresponding to "No problems (level 1) to some problems (level 2), and extreme problems" (level 3). The responses of the patients were converted to a scale ranging from -1 to $+1^{(16)}$.

\section{Vascular Quality of Life Questionnaire (VascuQol)}

The VascuQol is developed as a disease-specific questionnaire for patients with PAD. It contains 25 items (questions) subdivided into five dimensions: pain, symptoms, activities, social and emotional. Each question has a seven point response option, Patients" responses were converted to a scale ranging from 1 (worst possible score) to 7 (best possible score) ${ }^{(13)}$.

\section{Data analysis}

Data was analysed at the level of the symptomatic leg, defined as the leg in which treatment was performed. If the patient was treated on both legs or was treated conservatively, the symptomatic leg was defined as the leg with the most severe symptoms according to the patient at baseline. In case symptoms at baseline were comparable in both legs, we selected a leg with the lowest ABPI. When patients with critical ischemia could not complete a walking treadmill test due to severe pain complaints in their legs, the missing value for walking distance was imputed by the worst possible score. Missing items on returned questionnaires were imputed using 
the mean value of that variable. If questionnaires were not returned by patients, these patients were excluded from the respective analysis.

Because there is no gold standard for $\mathrm{QoL}$, an important assumption underlying the analysis was that disease severity is a major determinant of QoL. This implies that the construct validity of a QoL questionnaire is reflected by its ability to discriminate between severely and less severely diseased patients. In order to make this assumption more plausible patients with severe ca-morbidity (which is another important determinant of QoL) were excluded from analysis (17). Comorbidity was defined as a myocardial infarction within the previous 6 months, a cerebrovascular accident (CVA), or severe renall failure needing dialysis.

Moreover, the natural course of patients with critical ischemia is malignant due to high mortality in contrast to the often benign natural course for patients with claudication. As a result, the relevance of QoL measurements in patients with claudication and critical ischemia has different aspects. Therefore, the comparative validity of the questionnaires was assessed separately for patients with claudication and for patients with critical ischemia.

The cross-sectional construct validity was assessed for all three questionnaires. It was assumed that the cross-sectionall construct validity of a questionnaire is reflected by its ability to discriminate at baseline between patients with mild and severe disease by showing lower QoL scores in more severely diseased patients. Indicators for disease severity that were used were Rutherford classification, and treadmill walking distance. The median values of these indicators were used as cut-off points to classify patients into two groups of equal size with severe versus less severe disease. This approach was chosen, because it is statistically efficient.

Likewise, the longitudinal construct validity was assessed for all three questionnaires. It was assumed that the longitudinal construct validity of a questionnaire is reflected by its ability to capture change in QoL after treatment by showing larger changes in QoL scores in patients with larger changes in disease severity. Changes in indicators for disease severity (Rutherford classification, and treadmill walking distance) were calculated by subtracting the baseline score from the score measured at 6 months after the date of the diagnostic exam. Again, the median values of the changes in indicators of disease severity were used as cut-off point to classify patients into two groups of equal size with large improvement versus small or no improvement.

Both cross-sectional construct validity and longitudinal construct validity were compared between the three questionnaires. We present the mean QoL scores in the groups with severe versus less severe disease (cross-sectional construct validity) and the mean change in the groups with large improvement versus small or no improvement (longitudinal construct validity). The validity of the questionnaires was quantified by constructing receiver operating characteristic (ROC) curves and calculating areas under the curves (AUCS). The questionnaire with the highest AUC is superior in its ability to discriminate between severe and mild disease at baseline and to detect change during follow-up. The method of Hanley et al. was used to test statistical differences between the AUCs $(P<.05)^{(18)}$. 
The primary analysis consisted of comparing ALCs based on total scores on each questionnaire. For calculating the total score on the SF-36 we used the algorithm of Brazier et al. ${ }^{(19,20)}$. This algorithm uses 6 out of 8 dimensions and requires information on 11 of the original 36 items. In our study only 3 dimensions and 6 items were available. We selected four dimensions, because these have shown to be the most responsive for measuring changes in QoL after treatment of patients with $\mathrm{PAD}{ }^{[8,13,15)}$. Missing items in our study were set to zero in the algorithm of Brazier et al.

In additional analyses, we compared AUCs based on the score on dimensions measuring similar constructs such as physical functioning of the SF-36 and the dimension activities of the Vascuqol.

We also performed a subgroup analysis in patients with claudication without any co-morbid disease, excluding all patients with diabetes, CVA, myocardial

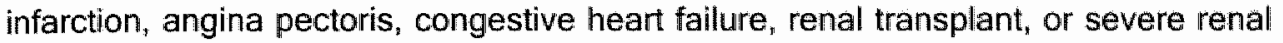
failure needing dialysis. This left 251 patients for analysis.

\section{Results}

At baseline, the VascuQol, SF-36 and Euroqol-5D were returned by 443 of 450 patients. In 7 patients the questionnaires were not returned owing to non-compliance $(n=4)$, comorbidity $(n=1)$, or death $(n=2)$. After six months follow-up, 386 patients returned questionnaires. No follow-up occurred in 64 patients because of noncompliance $(n=35)$, comorbidity $(n=11)$, or death $(n=18)$. All returned questionnaires had high completion rates. Completion rates for the individual dimensions varied from $98.7-99.5 \%$ (Euroqol-5D) to $98.7-99.7 \%$ (VascuQol) to $97.5-99.5 \%$ (SF-36).

Of the 386 patients who had returned their questionnaires after follow-up, 348 patients had intermittent claudication and 38 patients had critical ischemia. Table 1 shows the baseline characteristics of these patients. Figure 1 shows the number of patients who returned their questionnaires at follow-up and underwent conservative or interventional treatment. Of the 91 patients who underwent surgery, the time interval between date of surgery and returning their follow-up questionnaire was more than 2 months for 71 patients ( $78 \%$ ). 
Table 1 Baseline characteristics of patients who returned questionnaires after follow-up

\begin{tabular}{|c|c|c|c|c|}
\hline Characteristics of 386 patients & \multicolumn{2}{|c|}{ Claudication } & \multicolumn{2}{|c|}{ Critical ischemia } \\
\hline Number of pattents & \multicolumn{2}{|l|}{348} & \multicolumn{2}{|l|}{38} \\
\hline Male / female & \multicolumn{2}{|c|}{$230(66 \%) / 118(34 \%)$} & \multicolumn{2}{|c|}{$24(63 \%) / 14(37 \%)$} \\
\hline Age (mean;(SD)) & \multicolumn{2}{|l|}{$64(11)$} & \multicolumn{2}{|l|}{$65(12)$} \\
\hline \multicolumn{5}{|l|}{ Medical history } \\
\hline Tobacco use (ever/ never) & $325(93 \%)$ & $123(7 \%)$ & $35(92 \%)$ & $13(8 \%)$ \\
\hline Diabetes mellitus & 62 & $(18 \%)$ & 20 & $(53 \%)$ \\
\hline Hypertension & 162 & $(47 \%)$ & 21 & $(55 \%)$ \\
\hline Hyperlipidemia & $174^{*}$ & $(50 \%)$ & $16^{*}$ & $(42 \%)$ \\
\hline \multicolumn{5}{|l|}{ Renal disease } \\
\hline Mild renal insufficiency & $13^{*}$ & $(4 \%)$ & $3^{*}$ & $(8 \%)$ \\
\hline Renal transplant & 3 & $(1 \%)$ & 4 & $(11 \%)$ \\
\hline \multicolumn{5}{|l|}{ Cardiac disease } \\
\hline Congestive heart failure & 10 & $(3 \%)$ & 3 & $(8 \%)$ \\
\hline Myocardial infarction $>6$ months ago & 61 & $(18 \%)$ & 6 & $(16 \%)$ \\
\hline Angina pectoris & 36 & $(10 \%)$ & 2 & $(5 \%)$ \\
\hline Transient ischemic attack (TIA) & 20 & $(6 \%)$ & 3 & $(8 \%)$ \\
\hline Previous revascularisation & 126 & $(36 \%)$ & 17 & $(45 \%)$ \\
\hline \multicolumn{5}{|l|}{ Rutherford classification } \\
\hline \multicolumn{5}{|l|}{ None } \\
\hline \multicolumn{5}{|l|}{ Mild claudication } \\
\hline Moderate claudication & \multirow{5}{*}{$\begin{array}{l}38 \\
167 \\
141\end{array}$} & \multirow{2}{*}{\multicolumn{3}{|c|}{$\begin{array}{r}(11 \%) \\
(11 \%)\end{array}$}} \\
\hline Severe claudication & & & & \\
\hline Ischemic rest pain & & (48\%) & \multirow{2}{*}{$\begin{array}{l}17 \\
21\end{array}$} & $(45 \%)$ \\
\hline Critical ischemia with minor ulcer & & $(41 \%)$ & & $(55 \%)$ \\
\hline Critical ischemia major ulcer & & & 0 & $(0 \%)$ \\
\hline \multicolumn{5}{|l|}{ Exercise data at baseline (mean,(SD)) * } \\
\hline$A B P I$ at rest & 0.62 & $(0.19)$ & 0.52 & $(0.25)$ \\
\hline ABPI after exercise & 0.39 & $(0.23)$ & 0.38 & $(0.33)$ \\
\hline Walking distamce (meter) & 201 & (93) & 101 & $(110)$ \\
\hline
\end{tabular}

Note. Data are in numbers of patients and percentages are in between parentheses.

" For some patients information on these baseline characteristics was missing.

The percentages refer to the number of patients for whom data was available

\section{Cross-sectional Construct validity}

The construct validity was determined separately for patients with claudication and critical ischemia. Table 2 presents the mean values of all questionnaires after classifying patients into two groups based on the respective indicators of disease severity at baseline.

All questionnaires of patients with claudication and critical ischemia showed higher mean values of QoL in patients with less severe disease compared to patients with more severe disease irrespective of the indicators of disease severity (Table 2). 
Moreover, all QoL scores in critical ischemia patients were lower than the QoL scores in patients with claudication (Table 2). These results indicate that all three questionnaires meet the expectation that more severely diseased patients have lower scores on QoL.

A comparison of AUCs calculated from total scores of all three questionnaires resulted in only minor differences between these AUCs. These differences were not statistically significant (all $P>.05$ ) (Table 2). Likewise, the ROC curve in Figure 2 shows no differences between the AUCs after classifying claudication patients into two groups according to the Rutherford classification.

Additional analyses comparing the dimensions Activity (Vascuqol) with Physical functioning (SF-36) showed minor differences between the AUCs. However; the AUC for discriminating between claudication patients with higher versus lower than median walking distance was significantly higher for the SF-36 $(0.76)$ than for the VascuQol $(0.68)(P<.002)$.

The subgroup anallysis for claudication patients without any co-morbid disease showed similar results. 
Table 2 Cross-sectional construct validity

\begin{tabular}{|c|c|c|c|c|c|c|}
\hline \multirow[t]{3}{*}{ Mean OoL values } & \multicolumn{6}{|c|}{ PATIENTS WITH CLAUDICATION $N=386$} \\
\hline & \multicolumn{3}{|c|}{ Walking distance } & \multicolumn{3}{|c|}{ Rutherford classification } \\
\hline & $\leq 200 m^{*}$ & $>200 \mathrm{~m}^{*}$ & AUC & 4 4. & $1-3$ & AUC \\
\hline VascuQal & & $\therefore$ & 3 & $\sqrt{3+3}$ & 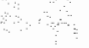 & $\therefore:$ \\
\hline Activity & 3.1 & 3.9 & 0.68 & 31 & 3.7 & 0.65 \\
\hline Symptoms & 4,4 & 47 & 0.59 & 4.3 & 4.6 & 0.57 \\
\hline Pain & 3.3 & 3.7 & 0.60 & 3.3 & 3.6 & 0.58 \\
\hline Social & 3.8 & 4.7 & 0.67 & 3.8 & 4.5 & 0.60 \\
\hline Emotional & 4.0 & 4.6 & 0.61 & 4.0 & 4.5 & 0.59 \\
\hline Total & 3.7 & 4.2 & 0.65 & 3.6 & 4.1 & 0.62 \\
\hline \multicolumn{7}{|l|}{$S F-36$} \\
\hline Physical functioning & 33 & 51 & $0.76^{1}$ & 34 & 46 & 0.66 \\
\hline Bodily pain & 44 & 52 & 0.61 & 43 & 51 & 0.61 \\
\hline Role physical functioning & 27 & 51 & 0.66 & 28 & 45 & 0.60 \\
\hline General health & 51 & 57 & 0.58 & 53 & 53 & 0.51 \\
\hline Total & 0.75 & 0.78 & 0.68 & 0.75 & 0.78 & 0.64 \\
\hline Eurogol-50 & 0.52 & 0.63 & 0.61 & 0.49 & 0.62 & 0.63 \\
\hline
\end{tabular}

$\mathrm{QoL}=$ quality of life; $\mathrm{N}=$ number of patients who returned their questionnaires at baseline. $\mathrm{AUC}=$ area under the curve.* For patients with claudication, the median value $(200 \mathrm{~m})$ of walking distance was used as cut-off value to define patients with severe disease $(\leq 200 \mathrm{~m})$ and mild disease $(>200 \mathrm{~m})$. \# The median value of Rutherford classification: 1-3 represents none to moderate claudication, 4 represents severe claudication. ${ }^{1}$ AUC for the SF-36 based on score on dimension physical activity is significantly higher than the AUC for the VascuQol based on score on the dimension activity $(P$ $<.002)$.

Figure 2 ROC Curve at baseline for patients with claudication

\section{Rutherford Classification}

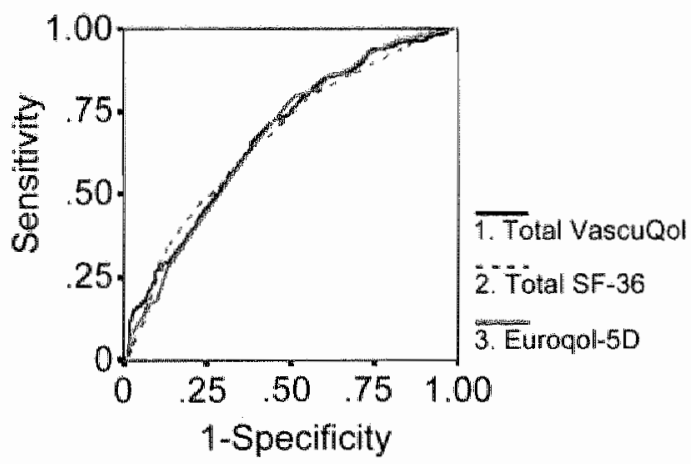

$P 1-2=.6$ is the $P$-value comparing AUCs of the VascuQol with the SF-36.

$P 1-3=.8$ is the P-value comparing AUCs of the VascuQol with the Euroqol-5D.

$P 2-3=.8$ is the $P$-value comparing AUCs of the SF-36 with the Euroqol-5D. 
Table 2

Continued

\begin{tabular}{|c|c|c|c|c|c|c|}
\hline \multirow[t]{3}{*}{ Mean QoL values } & \multicolumn{6}{|c|}{ PATIENTS WITH CRITICAL ISCHEMIA N= 57} \\
\hline & \multicolumn{3}{|c|}{ Walking distance } & \multicolumn{3}{|c|}{ Rutherford dassification } \\
\hline & $\leq 30 \mathrm{mt}$ & $>30 \mathrm{~m} t$ & AUC & $=6 \pm$ & $=5+$ & AUC \\
\hline \multicolumn{7}{|l|}{ VascuQd } \\
\hline Activity & 2.3 & 2.6 & 0.55 & 2.5 & 2.5 & 0.52 \\
\hline Symptoms: & 3.0 & 3.3 & 0.55 & 3.1 & 3.3 & 0.51 \\
\hline Pain & 2.3 & 2.1 & 0.46 & 2.4 & 2.0 & 0.36 \\
\hline Social & 2.7 & 3.1 & 0.57 & 2.9 & 3.0 & 0.48 \\
\hline Emotional & 2.9 & 3.4 & 0.59 & 3.1 & 3.5 & 0.56 \\
\hline Total & 2.9 & 29 & 0.57 & 2.8 & 2.8 & 0.52 \\
\hline \multicolumn{7}{|l|}{$S F-36$} \\
\hline Physical functioning & 28 & 31 & 0.54 & 27 & 31 & 0.56 \\
\hline Bodily pain & 29 & 34 & 0.61 & 31 & 35 & 0.58 \\
\hline Role physical funct & 21 & 16 & 0.46 & 20 & 17 & 0.46 \\
\hline General health & 38 & 38 & 0.48 & 38 & 37 & 0.46 \\
\hline Total & 0.71 & 0.73 & 0.57 & 0.71 & 0.73 & 0.59 \\
\hline Euroqot-5D & 0.29 & 0.43 & 0.62 & 0.31 & 0.40 & 0.57 \\
\hline
\end{tabular}

†For patients with critical ischemia the median value of walking distance was $30 \mathrm{~m}$.

¥ For patients with critical ischemia the median value of Rutherford classification was

Rutherford category 6 (patients with critical ischemia with minor ulcer, lie. the group with severe disease) and Rutherford category 5 (patients with ischemic rest pain, i.e. the group with "mild disease"). Role physical funct $=$ Role physical functioning dimension of the SF-36

\section{Longitudinal construct validity}

Patients with claudication were separated from critical ischemia patients, and Table 3 shows the mean values of change in QoL for patients with large improvement versus patients with small or no improvement at 6 months after imaging workup. All three questionnaires had higher mean values for change in $\mathrm{QoL}$ in patients with larger improvement compared to patients with small to no improvement. This trend was observed irrespective of which indicator of disease severity is considered.

To determine which questionnaire detected the largest change after follow-up, ROC curves and AUCs were calculated based on change in the total scores (Table 3 and Figure 3). AUCs for the Vascuqol were higher than the AUCs for the SF-36, which was significant for discriminating between claudication patients with more and less than median change in Rutherford classification (AUCs 0.82 versus $0.67, P<$ .001) (Table 3). The group of critical ischemia patients was too small to demonstrate statistical differences. 
Table 3 Longitudinal construct validity after 6 moniths follow-up

\begin{tabular}{|c|c|c|c|c|c|c|c|}
\hline \multirow[t]{3}{*}{ Mean change $(\Delta)$ in $\mathrm{OoL}$} & \multicolumn{7}{|c|}{ PATIENTS WITH CLAUDICATION } \\
\hline & \multicolumn{3}{|c|}{$\Delta$ Walking distance ${ }^{*}$} & \multicolumn{4}{|c|}{$\Delta$ Rutherford classification } \\
\hline & $\leq 7 m^{*}$ & $>7 \mathrm{~m}^{*}$ & ALC & $\leq 0$ & $\geq 1 \#$ & AUC & \\
\hline \multicolumn{8}{|l|}{ Vasculol } \\
\hline$\triangle$ Activity. & 0.9 & 1.4 & 0.58 & 0.1 & 1.8 & 0.82 & 3 \\
\hline$\Delta$ Symptoms & 0.6 & 1.0 & 0.59 & 0.1 & 1.2 & 0.75 & \\
\hline$\triangle$ Pain & 0.9 & 1.4 & 0.58 & 0.3 & 1.8 & 0.80 & \\
\hline$\triangle$ Social & 0.6 & 1.0 & 0.59 & 0.0 & 1.3 & 0.72 & \\
\hline$\Delta$ Emotionall & 0.6 & 1.1 & 0.60 & 0.0 & 1.4 & 0.79 & \\
\hline$\Delta$ Total & 0.8 & 1.2 & 0.60 & 0.1 & 1.5 & 0.82 & $t_{2} 2$ \\
\hline \multicolumn{8}{|l|}{$S F-36$} \\
\hline$\triangle$ Physical functioning & 11 & 18 & 0.57 & 1 & 22 & 0.77 & \\
\hline$\Delta$ Bodily pain & 8 & 14 & 0.57 & -1 & 19 & 0.73 & \\
\hline$\Delta$ Role phys functioning & 13 & 17 & 0.55 & 2 & 21 & 0.63 & \\
\hline$\Delta$ General health & -1 & 0 & 0.51 & -3 & 1 & 0.56 & \\
\hline$\Delta$ Total & 0.01 & 0.02 & 0.53 & 0.00 & 0.03 & 0.67 & \\
\hline$\triangle$ Euroqol-5D & 0.06 & 0.11 & 0.55 & -0.02 & 0.13 & 0.68 & \\
\hline
\end{tabular}

$\mathbb{N}=$ number of patients who returned their questionnaires after 6 months follow-up.

"The median value 7 and $3 \mathrm{~m}$ of the change in walking distance was used as cut-off value to define patients with little or no improvement $(<7$ and $3 \mathrm{~m})$ and large improvement $(>7$ and $3 \mathrm{~m})$ for patients with claudication and critical ischemia, respectively. The change in walking distance for the group with large improvement seems small, due to the low median walues. However, for the group with large improvement the mean change in walking distance was 87 and 142 meter for patients with claudication and critical ischemia, respectively.

\# Patient group with an upward shift by one of more $(z 1)$ clinical categories of the Rutherford classification.

Figure 3 ROC curve after follow-up for patients with claudication.

\section{Change in Rutherford Classification}

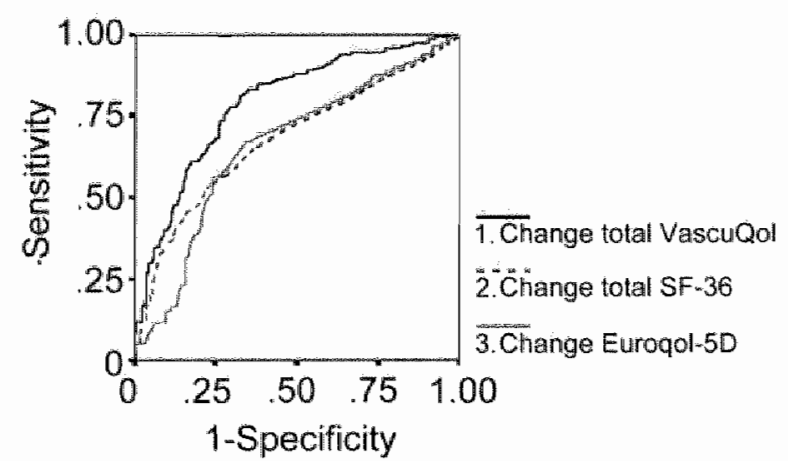

$P 1-2<.001$ is the $P$-value comparing the AUCs of the VascuQol with the SF-36.

$P 1-3<.001$ is the $P$-value comparing the AUCs of the VascuQol with the Euroqol-5D.

$P 2.3=.7$ is the P-value comparing the AUCs of the SF.36 with the Euroqol-5D. 
Table 3 Continued

Mean change $(\Delta)$ in $\mathrm{QoL}$

PATIENTS WITH CRITICAL ISCHEMIA N $=38$

$\triangle$ Walking distance*

$\triangle R$ Rutherford classification

$\leq 3 m^{*}>3 m^{*} \quad$ AUC $\leq 3 \quad 24+\quad$ AUC

VascuQol

$\triangle$ Activity

$0.7 \quad 1.6$

0.72

0.4

1.4

0.74

$\triangle$ Symptoms

1.4

2.2

0.67

0.8

2.0

0.78

$\triangle$ Pain

1.4

2.7

0.76

0.7

2.2

0.83

$\triangle$ Social

0.9

0.62

0.5

0.72

$\triangle$ Emational

$0.4 \quad 1.4$

0.76

0.2

0.74

$\triangle$ Total

0.9

0.76

0.5

1.1

$0.81^{2}$

$S F-36$

$\triangle$ Physical functioning

$\triangle$ Bodily pain

$\Delta$ Role phys functioning

$\triangle$ General health

$\triangle$ Total

$\begin{array}{rr}2 & 11 \\ 18 & 27 \\ 0 & 23 \\ -3 & 4 \\ 0.01 & 0.05 \\ 0.11 & 0.37\end{array}$

0.66

0.52

0.69

0.69

$\triangle$ Euroqol-5D

0.60

-4
2
2
-6
0.02
0.18

1.6

0.68

$\begin{array}{ll}27 & 0.77 \\ 18 & 0.58\end{array}$

$2 \quad 18 \quad 0.58$

$\begin{array}{ccc}-6 & 3 & 0.68\end{array}$

0.74

0.04

0.63

0.18

0.51

‡ Patient group with an upward shift by four of more $(\geq 4)$ clinical categories of the Rutherford classification. 'AUC for the VascuQol based on change in total score is significantly higher than the AUC for the SF-36 based on change in total score $(\mathcal{A} .05)$.

${ }^{3}$ AUC for the VascuQol based on change in total score is significantly higher than the AUC based on change in score on the Euroqol-5D $(P<.05)$.

${ }^{3}$ AUC for the VascuQol based on change in score on dimension activity is significantly higher than the AUC for the SF-36 based on change in score on the dimension physical activity $(P<.05)$.

The AUCs for the VascuQol were also higher than the AUCs for the Euroqol5D. Significant differences in AUCs were found for discriminating between more and less than median change in Rutherford classification for both patients with claudication (0.82 versus 0.68$)$ and patients with critical ischemia $(0.81$ versus 0.51 ) $(P<.003)$. This is also expressed in Figure 3, which shows a higher ROC curve for the VascuQol compared to ROC curves for the SF-36 and Euroqol-5D, after classifying claudication patients based on changes in Rutherford classification.

In additional analyses, comparing the dimensions Activity (Vascuqol) with Physical functioning (SF-36), the AUCs for the VascuQol were higher compared to the AUCs for the SF-36 (Table 3). The AUC for the Vascuqol (0.82) for discriminating between claudication patients with more and less than median change in Rutherford classification was significantly higher than the AUC for the SF-36 (0.77) $(P=.002)$.

The subgroup analysis for claudication patients without any co-morbid disease showed similar results. 


\section{Discussion}

The present study shows that the generic and the disease-specific questionnaires performed equally well in discriminating severe from mild disease at baseline in both patients with claudication and critical ischemia (Table 2). However, the diseasespecific questionnaire (VascuQol) was better in discriminating a large versus a small change in disease severity after follow-up than the generic questionnaires (SF-36 and the Euroqol-5D) (Table 3) for both patients with claudication and critical ischemia. These results suggest that the VascuQol is a better questionnaire than the $S F-36$ or Euroqol-5D to detect change in $Q 0 L$ in patients with PAD.

Our findings are consistent with other studies, whilch showed that the disease severity of PAD at baseline and change in disease severity are reflected by $\mathrm{QOL}$ scores as measured by VascuQol, SF- 36 and Euroqol-5D ${ }^{(4,13,21,22)}$. However, the unique feature of our study is the statistically grounded comparison of a diseasespecific questionnaire with generic questionnaires, with respect to their ability to assess QoL at baseline and to capture change in QoL during follow-up.

A frequently applied method for comparing questionnaires is to calculate correlation coefficients ${ }^{(4,13,21,22)}$. However, we opted for calculating areas under the ROC curves, because this provides a method to quantify the differences between the questionnaires with respect to cross-sectional and longitudinal construct validity, and these differences can easily be tested.

The reason for the overall limited use of disease-specific questionnaires, might be that these questionnaires are relatively new and have undergone a limited validation process ${ }^{(12)}$. One recommended disease-specific questionnaire, the CLAU$S$, has often been used in trials using specific medication, but it measures only QoL in patients with intermittent claudication ${ }^{(23-25)}$. We preferred to use the VascuQol, because both patients with intermittent claudication and patients with critical ischaemia were included in our study.

A major problem in comparing the validity of QoL questionnaires is the lack of a gold standard. The approach in this study is driven by the assumption that disease severity is a major determinant of quality of life. However, it might be argued that "objective" disease severity is not always directly related to loss of quality of life, and that other parameters like the patients' expectations of life and co-morbidity are also major determinants of QoL. To avoid confounding by co-morbidity we restricted the study population to patients without severe co-morbidity. We think, that the assumption is valid that differences in differences in disease severity in such a population are reflected by differences in QoL.

A limitation of this present study was that not all dimensions of the SF-36 questionnaire were used. Selection of dimensions may have induced loss of information and, consequiently a sub-optimal calculation of a total score on the SF36. Our reason for selecting specific dimensions was that the length of the SF-36 has been shown to be unfavorable for full completion of all questions by the patient ${ }^{(26)}$. In cur opinion, full completion of a selected number of dimensions was more important than poor completion of all dimensions in the SF-36 questionnaire. Indeed, the completion rate of the four dimensions of SF-36 was substantially higher (range, 98$99 \%)$ than reported in previous studies which used all dimensions SF-36 (70- 
$91 \%)^{(26,27)}$. We used the dimensions physical functioning, role physical functioning, bodily pain and general health because these items have proved to be the most responsive in detecting changes after follow-up in patients with PAD ${ }^{(8,13,15)}$. The dimensions and items that were excluded, have previously been shown to change little during follow-up of patients with PAD ${ }^{(8,13,15)}$. Based on the results of these studies, we assumed that the excluded items would remain constant in our population and may not affect the change in the total score of the SF-36 ${ }^{(8,13,15)}$.

Another limitation of our study was the substantial proportion of patients with missing data after follow-up, because 64 patients had not returned their questionnaire. It is not to be expected that the missing data influenced the outcome of the comparison between the questionnaires.

Moreover, the questionnaires were returned for practical reasons by mail, instead of administration of the SF-36 by a third party. However, we had high completion rates of the returned questionnaires, indicating that patients had no difficulties for completing questionnaires.

Furthermore, it must be noted that the results of this study pertain only to a subgroup of patients with PAD, namely patients who needed imaging work-up to determine treatment policy. From a clinical viewpoint, the question which type of questionnaire has the best ability to detect changes in QoL after treatment, is most relevant for this subgroup. The results of this study, however, may not be generalized to patients with uncomplicated claudication who do not need an image work-up.

Calculation of the total score of the SF-36 has been criticized in the literature because it has not been thoroughly validated in patients, although some studies showed that the total score of the SF-36 is a valid measure ${ }^{(28-30)}$. Comparison of total scores of different questionnaires might be more valid to determine which questionnaire is preferred instead of comparing several not always corresponding health dimensions. Although our calculation of the total score of the SF-36 was difficult because not all items were included in our study, the AUCs based on the total score of the SF-36 were similar to the AUCs based on the scores in the four individual dimensions of the SF-36 (Tables 2 and 3). Furthermore, to verify our results of comparing AUCs based on total scores of all questionnaires, we compared in additional analyses the AUCs based on the score of the most responsive dimension of the SF-36 ("physical activity") with the corresponding dimension of the VascuQol ("activities"). These additional analyses showed similar results as the comparison of AUCs based on total scores (Tables 2 and 3).

\section{Practical application}

The treatment of patients with PAD is, or should be, predominantly aimed at improving $\mathrm{QOL}{ }^{(7-11)}$. QOL is important to evaluate different treatment strategies and their cost-effectiveness. Therefore, assessment of $\mathrm{QoL}$ could be a stronger basis for evaluating effects of treatment than the traditional clinical outcomes such as ABPI. As was demonstrated in other studies changes in ABPI correlate poorly with changes in QoL scores ${ }^{(5,13,15)}$. This finding indicates that a frequently used "hard" measure of outcome does not always reflect the patient"s perceived QoL. 
Currently, most patients with claudication are treated conservatively, and only those patients who have severe symptoms according to the opinion of the surgeon are considered for interventional treatment. Hicken et al. showed that surgeons misjudged their patients' QoL in everyday clinical practice ${ }^{(2)}$. Consequently, the surgeons might assign interventional treatment to the wrong patients. Standardised assessments of QoL by means of questionnaires can help surgeons decide which patients are eligible for interventional treatment. Based on the assumption that disease severity is an important determinant of quality of life, the results of our study favor the use of the VascuQol due to its the superior construct validity compared to the SF-36 and Eurogol-5D for assessing changes in QoL in patients with PAD.

\section{Conclusions}

All three questionnaires VascuQol, SF-36 and Euroqol-5D performed equally well in assessing QoL at baseline in patients with PAD. The disease-specific VascuQol is superior to the generic questionnaires SF-36 and Euroqol-5D with respect to the detection of changes in QoL after follow-up. The VascuQol is the preferred questionnaire as outcome measure for $\mathrm{QoL}$ in future trials and clinical follow-up of patients with PAD. 


\section{References}

1. Khaira $H S_{\mathrm{k}}$ Hanger $R$, Shearman $C P$. Quality of life in patients with intermittent claudication. Eur J Vase Endovasc Surg 1996;11(1):65-69.

2. Hicken $G J$, Lossing $A G$, Ameli $M$. Assessment of generic health-related quality of life in patients with intermittent claudication. Eur J Vasc Endovasc Surg 2000;20(4);336-341.

3. Pell JP. Impact of intermittent claudication on quiality of life. The Scottish Vascular Audit Group. Eur J Vasc Endovasc Surg 1995;9(4):469-472.

4. de Vries $S$, Kuipers $W$, Hunink M. Intermittent claudication: Symptom severity versus health values. Journal of Vascular Surgery 1998;27(3):422-430.

5. Chetter IC, Dolan P, Spark JI, Scott DJ, Kester RC. Correlating clinical indicators of lower-limb ischaemia with quality of life. Cardiovasc Surg 1997:5(4):361-366.

6. Fowkes $F G$, Gillespie $\mathbb{N}$. Angioplasty (versus non surgical management) for intermittent claudication. Cochrane Database Syst Rev 2000(2):CD000017.

7. Hiatt WR, Hirsch AT, Regensteiner JG, Brass EP. Clinical Trials for Claudication : Assessment of Exercise Performance, Functional Status, and Clinical End Points. Circulation 1995;92(3):614-621.

8. Bosch JL, van der Graaf $Y$, Hunink MG. Health-related quality of life after angioplasty and stent placement in patients with iliac artery occlusive disease: results of a randomized controlled clinical trial. The Dutch lliac Stent Trial Study Group. Circulation 1999;99(24):3155-3160.

9. Cassar $K_{\text {, }}$ Bachoo $\mathrm{P}$, Brittenden J. The Effect of Peripheral Percutaneous Transluminal Angioplasty on Quality of Life in Patients with Intermittent Claudication. European Journal of Vascular and Endovascular Surgery 2003;26(2):130-136.

10. Chetter IC, Spark JI, Kent PJ, Berridge DC, Scott DJ, Kester RC. Percutaneous transluminal angioplasty for intermittent claudication: evidence on which to base the medicine. Eur J Vasc Endovasc Surg 1998;16(6):477-484.

11. Dormandy JA, Rutherford RB. Management of peripheral arterial disease (PAD). TASC Working Group. TransAtlantic Inter-Society Concensus (TASC). J Vasc Surg 2000;31(1 Pt 2):S1-S296.

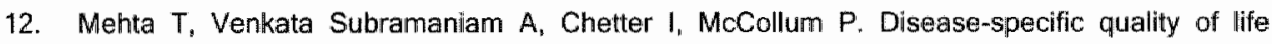
assessment in intermittent claudication: review. Eur J Vasc Endovasc Surg 2003:25(3):202-208.

13. Morgan MB. Crayford $T$. Murrin B, Fraser SC. Developing the Vascular Quality of Life Questionnaire: a new disease-specific quality of life measure for use in lower limb ischemia. J Vasc Surg 2001;33(4):679-687.

14. McHorney $\mathrm{CA}_{4}$ Ware $\mathrm{JE}_{\mathrm{s}} \mathrm{Jr}_{\mathrm{r}}$, Lu JF, Sherboume CD. The MOS 36-item Short-Form Health Survey (SF-36): III. Tests of data quality "scaling assumptions, and reliability across diverse patient groups. Med Care 1994;32(1):40-66.

15. Currie IC, Wilson YG, Baird RN, Lamont PM. Treatment of intermittent claudication: the impact on quality of life. Eur J Vasc Endovasc Surg 1995:10(3):356-361.

16. Dolan P. Modeling valuations for EuroQol health states. Med Care 1997:35(11): 1095-1108.

17. Breek J, Hamming $\downarrow$, De Vries J, Henegouwen D, van Heck G. The impact of walking impairment, cardiovascular risk factors, and comorbidity on quality of life in patients with intermittent claudication. Journal of Vascular Surgery 2002;36(1):94-99.

18. Hanley JA, McNeil BJ. A method of comparing the areas under receiver operating characteristic. curves derived from the same cases. Radiology 1983;148(3):839-843.

19. Brazier $J$, Roberts $J$, Deverill M. The estimation of a preference-based measure of health from the SF-36. J Health Econ 2002;21(2):271-292. 
20. Brazier $J$, Usherwood $T$, Harper $R$, Thomas $K$. Deiriving a preference-based single index from the UK SF-36 Health Survey. J Clin Epidemiol 1998;51(11):1115-1128.

21. Chetter IC, Spark JI, Dolan P, Scott DJ, Kester RC. Quality of life analysis in patients with lower limb Ischaemia: suggestions for European standardisation. Eur J Vasc Endovasc Surg $1997,3(6): 597-604$.

22. Wann Hansson CC, Rahm $H_{3}$ I, Risberg $\mathrm{BB}_{3}$ Klevsgard RR. A comparison of the Nottingham Health Profile and Short Form 36 Health Survey in patients with chronic lower limb ischaemia in a longitudinal perspective. Heatth Qual Life Outcomes 2004,2(1):9:

23. Spengel $F$, Clement $D_{1}$ Boccalon $H$, Llard $F$, Brown $T$, Lehert $P$. Findings of the Naftidrofuryl in Quality of Life (NIQOL) European study program. Int Angiol 2002;21(1):20-27.

24. D'Hooge D, Lehert $P$, Clement DL. Naftidrofuryl in quality of life (NIQOL). A. Belgian study. Int Angiol 2001;20(4):288-294.

25. Spengel $F$, Brown TM, Poth J, Lehert P. Naftidrofuryl can enhance the quality of life in patients with intermittent claudication. Vasa 1999;28(3):207-212.

26. Brazier JE, Watters S.J, Nicholl JP, Kohler B. Using the SF-36 and Euroqol on an elderly population. Qual Life Res 1996;5(2):195-204.

27. Harper R, Brazier JE, Waterhouse JC, Walters SJ, Jones NM, Howard P. Comparison of outcome measures for patientis with chronic obstructive pulmonary disease (COPD) in an outpatient setting. Thorax 1997;52(10):879-887.

28. Lee TA, Hollingworth $W$, Sullivan $S D$. Comparison of directly elicited preferences to preferences derived from the SF-36 in adults with asthma. Med Decis Making 2003;23(4):323-334.

29. Conner-Spady $B$, Suarez-Almazor ME. Variation in the estimation of quality-adjusted life-years by different preference-based instruments. Med Care 2003;41(7):791-801.

30. Longwarth L, Bryan S. An empirical comparison of EQ-5D and SF-6D in liver transplant patienis. Health Econ 2003;12(12):1061-1067. 


\section{CHAPTER 4}

Clinical and Cost Comparisons of Duplex Ultrasound and Contrast-Enhanced Magnetic Resonance Angiography in Patients with Peripheral Arterial Disease: a Multicenter Randomized Trial

Radiology 2005; Accepted

Marianne de Vries, Rody Ouwendijk, Karin Flobbe, Patricia J. Nelemans, Alphons G. Kessels, GeertWillem H. Schurink, J. Adam van der Vliet, Frans M.J. Heijstraten, Philippe W.M. Cuypers, Lucien E.M. Duijm, Jos M.A.van Engelshoven, M.G. Myriam Hunink, Michiel W. de Haan. 


\section{ABSTRACT}

\section{Purpose}

To determine clinical and economic consequences of replacing duplex ultrasound (DUS) by contrast-enhanced MR angiography (CE-MRA) for the initial imaging workup of patients with peripheral arterial disease (PAD).

\section{Materials and Methods}

The study was approved by the institutional review board, and all patients signed written informed consent. In a randomized multicenter trial, patients with PAD who needed imaging workup and had an ankle brachial pressure index $(A B P I)<0.90$, were recruited by vascular surgeons between January 2002 and September 2003. Patients were randomly assigned to CE-MRA or DUS, the new versus the current imaging workup. Primary outcome measure were costs. Secondary outcomes included therapeutic confidence, change in disease severity and change in quality of life (QOL) assessed during 6 months follow-up. Indicators for disease severity were Rutherford classification, treadmill walking distance, $A B P I$ at rest, and $A B P I$ after exercise. QoL was assessed by Short-Form 36, EuroQol-5D and VascuQol. All costs of (additional) imaging, therapeutic interventions and outpatient visits were calculated from a hospital perspective. Data were evaluated with Student thest and multivariable linear regression analysis.

\section{Results}

At 6 months, 352 patients (239 (68\%) men, $113(32 \%)$ women; mean age 65 years) were analyzed. Use of CE-MRA reduced the number of additional vascular imaging procedures by $42 \%$ compared to DUS, and CE-MRA was associated with a higher therapeutic confidence. Total diagnostic costs of CE-MRA were $€ 167$ higher than the costs of DUS. This difference was statistically significant $(P<.001)$. No statistically significant differences were found for the total costs, change in disease severity and change in QoL between DUS and CE-MRA (all P. 05).

\section{Conclusion}

The choice between DUS and CE-MRA for the initial imaging workup for patients with PAD depends on the local expertise with these techniques and cost considerations relevant to the local setting. Replacing DUS by CE-MRA for the initial imaging workup of patients with PAD reduces the need for additional imaging, albeit the total diagnostic costs are higher. 


\section{Introduction}

A challenge in the imaging workup for patients with peripheral arterial disease (PAD) is choosing the best imaging workup which provides all information and is costeffective. Peripheral arterial disease (PAD) of the lower legs is a common and disabling disease $(1,2)$. Revascularisation strategies for patients with PAD require assessment by means of imaging workup. If revascularisation is being considered, most centres in the Netherlands use duplex ultrasound (DUS) as the initial imaging modality, sometimes followed by digital subtraction angiography (DSA) $(3,4)$. Only a few centres use contrast-enhanced MR angiography (CE-MRA) as the initial imaging modality for detecting stenoses in patients with PAD.

DUS is a well-established non-invasive modality with good sensitivity and specificity (5), and its performance can be further improved by the addition of functional (colour flow) imaging (6). However, DUS is operator-dependent, and does not provide an easy-to-interpret image ("roadmap") of the vascular system which is useful for treatment planning. Finally, it is often difficult to depict the infra-popliteal vessels.

Some studies $(7,8)$ have preferred CE-MRA to DUS, for several reasons. An often mentioned argument is that CE-MRA does produce a "roadmap" of the arteries, making CE-MRA a more effective tool for treatment planning compared to DUS. Moreover, some studies showed that CE-MRA is a more effective tool for treatment planning compared to DUS and even to DSA (8-11). In addition, a meta-analysis by Visser et al. showed that CE-MRA has better discriminatory power than DUS (7). On the other hand, CE-MRA has the disadvantage that it requires a contrast agent, and some patients have contraindications to undergo MR imaging.

Despite favorable reports on CE-MRA, many centres use this technique only as an additional exam instead of an initial imaging exam. It is currently unknown whether CE-MRA or DUS is most cost-effective as initial imaging test in everyday clinical practice. Thus, the purpose of our study was to determine the clinical and economic consequences of replacing DUS by CE-MRA for the initial imaging workup of patients with PAD.

\section{Material and Methods}

\section{Setting, Patients and Randomization}

The present study was a prospective multicenter study in which patients with PAD were randomized between CE-MRA and DUS to compare imaging workup strategies (the new versus the current imaging workup, respectively). Two university hospitals (Maastricht University Medical Center, Maastricht, and University Medical Center St Radboud, Nijmegen) and one general hospital (St Catharina Hospital, Eindhoven) all in the Netherlands participated in the study. Between January 2002 and September 2003 all patients with $\mathrm{PAD}$, who were referred by the vascular surgeons for imaging workup to evaluate the feasibility and choice of revascularisation procedure, were eligible for enrolment. The study population consisted of patients with intermittent 
claudication or critical ischaemia and an ankle brachial pressure index (ABPI) $<0.90$. Patilents were excluded if they needed imaging workup within 3 days, had contraindications for MR angiography, and already had had a previous imaging workup indicating that revascularisation was needed.

The study was approved by each hospital institutional review board, and all patients signed written informed consent prior to randomization. Randomization was performed centrally and took place through the Trial Coordinating Center by telephone. The random allocation scheme used a computer-generated black design with a block size of 8 . Patients, the referring vascular surgeons and research assistants were all unaware of the randomization sequence. Given the nature of the procedure, patients and physicians were not blinded to the assigned imaging workup.

\section{Imaging and Evaluation}

Each participating hospital was equipped with state-of the art MR-scanners (1.5 Tesla), and DUS equipment. Due to variety of the manufacturers and models of imaging equipment each hospital was allowed to use the imaging protocols, which it considered to be optimal (Table 1). Radiologists interpreted images as part of their normal workflow:

In all three hospitals, DUS was performed by experienced ultrasonographers (range of experience; 7 to 15 years). After randomisation the referring vascular surgeon determined the extent of the DUS examination (aortoiliac and 1 or femoropopliteal arteries only, or the need for imaging of the crural arteries), based on the history and findings on physical examination of the patient.

In all three hospitals, DUS was performed by experienced ultrasonographers.

The referring vascular surgeon determined the extent of the DUS examination (aortoiliac and / or femoropopliteal arteries only, or the need for imaging of the crural arteries), based on the history and findings on physical examination of the patient.

\section{Outcome Measures}

The outcome measures were therapeutic confidence, change in disease severity, change in quality of life (QoL), and costs, during 6 months follow-up. Provided that improvement in QoL was equal for the CE-MRA and DUS group, our primary outcome measures were costs. Consequently, measuring patients Q Q was also an important outcome measure.

During a weekly vascular conference, the therapeutic confidence was assessed by the radiologists' and vascular surgeons" confidence with respect to their ability to make a therapeutic decision based on the performed imaging modality and was rated on a scale of $0-10(0=$ no confidence, $10=$ extremely confident to take a treatment decision). The final confidence score was based on a consensus between the radiologist's and vascular surgeon's opinion. At each participated hospital three radiologists and four vascular surgeons were involved regarding the therapeutic confidence assessment. Furthermore, it was recorded whether additional vascular 
imaging was necessary and performed. The recommended treatment was recorded as well.

Indicators for disease severity were Rutherford classification, treadmill walking distance, $\mathrm{ABP} \mid$ at rest, and $\mathrm{ABP}$ after exercise, which were determined at baseline and 6 months after imaging workup (measurements were performed by the technicians). Disease severity was assessed by the vascular surgeon who treated the patient.

Table 1

\begin{tabular}{|c|c|c|c|c|}
\hline & & Eindhoven & Maastricht & Nijmegen \\
\hline \multicolumn{5}{|c|}{ DUs imaging } \\
\hline \multicolumn{2}{|c|}{ Manufacturer DUS II } & Siemens & Aloka & Phillps \\
\hline \multicolumn{2}{|c|}{ Aortoiliac transducer } & 4 and $5 \mathrm{MHz}$ & 3.5 and $5 \mathrm{MH}-\mathrm{z}$ & 2-4 MHz \\
\hline \multicolumn{2}{|c|}{ Upper + lower legs transducer } & 5 and $7.5 \mathrm{MHz}$ & $7.5 \mathrm{MHz}$ & $4-7 \mathrm{MHz}$ \\
\hline \multicolumn{5}{|c|}{ Sienose Severity: $¥$} \\
\hline $1=$ & $0-19 \%$ & $P S V=50-130 \mathrm{~cm} / \mathrm{sec}$ & PSVR $<1.5$ & PSV $45-150 \mathrm{~cm} / \mathrm{s}$ \\
\hline $2=$ & $20-49 \%$ & PSV $\uparrow$ of $30 \%$ & $1.5>$ PSVR $<2.5$ & $1.5>$ PSVR $<2.5$ \\
\hline $3=$ & $50-75 \%$ & $\begin{array}{l}\text { PSV } \uparrow \text { of } 50 \% \text { and } \\
\text { spectral broadening } \uparrow\end{array}$ & $\begin{array}{l}\text { PSVR } \geq 2.5 \text { and } \\
E D V<60 \mathrm{~cm} / \mathrm{s}\end{array}$ & $\begin{array}{l}\text { PSVR } \geq 2.5^{*} \text { and } \\
E D V<60 \mathrm{~cm} / \mathrm{s}\end{array}$ \\
\hline $4=$ & $75-99 \%$ & $\begin{array}{l}\mathrm{PSV} \uparrow \text { of } 50 \% \text { and } \\
\mathrm{EDV}>20 \mathrm{~cm} / \mathrm{s}\end{array}$ & $\begin{array}{l}P S V R \geq 2.5 \text { and } \\
E D V>60 \mathrm{~cm} / \mathrm{s}\end{array}$ & $\begin{array}{l}\text { PSVR } \geq 2.5 \text { and } \\
E D V>60 \mathrm{~cm} / \mathrm{s}\end{array}$ \\
\hline $5=$ & Occlusion & no signal & no signal & no signal \\
\hline \multicolumn{5}{|c|}{ MR imaging } \\
\hline \multicolumn{2}{|c|}{$\begin{array}{l}\text { Manufacturer MR sicanner } \\
\text { * fiield strength }\end{array}$} & $\begin{array}{l}\text { Philips } 1.5 \mathrm{~T} \\
\text { (release } 8.1 .3 \text { ) }\end{array}$ & $\begin{array}{l}\text { Philips } 1.5 \mathrm{~T} \\
\text { (release } 8.2 \text { ) }\end{array}$ & $\begin{array}{l}\text { Siemens } 1.5 \mathrm{~T} \\
\text { (release VB } 33 \mathrm{G} \text { ) }\end{array}$ \\
\hline \multicolumn{2}{|l|}{ Coil +} & $\begin{array}{l}\text { Quadrature body } \\
\text { gadoteridol }\end{array}$ & $\begin{array}{l}\text { Peripheral vascular } \\
\text { gadolinium DTPA }\end{array}$ & $\begin{array}{l}\text { Body phased array } \\
\text { gadolinium DTPA }\end{array}$ \\
\hline \multicolumn{2}{|c|}{$\begin{array}{l}\text { Manner of contrast injection \# } \\
\text { Bolus arrival }+\dagger\end{array}$} & $\begin{array}{l}1 \text { injection of } 40 \mathrm{ml} \\
\text { BolusTrak }\end{array}$ & $\begin{array}{l}1 \text { injection of } 35 \mathrm{ml} \\
\text { BolusTrak }\end{array}$ & $\begin{array}{l}3 \times 15 \mathrm{ml} \\
\text { Test bolus }\end{array}$ \\
\hline \multicolumn{2}{|c|}{ Injection rate (mi/sec) phase } & $\begin{array}{l}\text { First } 20 \mathrm{ml} \text { at } 0.6 \mathrm{ml} / \mathrm{s} \\
\text { last } 20 \mathrm{ml} \text { at } 0.3 \mathrm{ml} / \mathrm{s}\end{array}$ & $\begin{array}{l}\text { First } 15 \mathrm{ml} \text { at } 1.8 \mathrm{ml} / \mathrm{s} \\
\text { last } 20 \mathrm{ml} \text { at } 0.6 \mathrm{ml} / \mathrm{s}\end{array}$ & $2.5 \mathrm{mi} / \mathrm{sec}$ \\
\hline \multicolumn{2}{|c|}{ Name of MR protocol + t } & MobiTrak & Flexible parameters & 3 separate injections \\
\hline
\end{tabular}

\#PSVR = peak systolic velocity ratio, EDV = end diastolic velocity. "A stenose was $>50 \%$ when the PSV $>2.0$ for patients with a bypass in the upper legs. $\dagger$ The peripheral vascular coil is a dedicated, $129 \mathrm{~cm}$ long, 12-element phased array coil (Philips Medical Systems, Best, The Netherlands) that was used for all three stations. \#All injections were done with a remote controlled MR compatible injection system (Medrad Specris, Indianola, PA USA). †† Appropriate start of CE-MRA acquisition was determined using real time bolus monitoring software ((BolusTrak, Philips Medical Systems, Best, The Netherlands), or with (Test Bolus, Siemens, Siemens Medical Systems, Erlangen, Germany)).

\# To obtain MR arteriograms of the entire peripheral arterial tree, Hospital 1 uses a commercially available software package (MobiTrak. Philips Medical Systems, Best, The Netherlands).

MobiTrak uses for all three stations the same fixed imaging parameters. In some patients hospitall 1 used flexible parameters protocol. To improve spatial and temporal resolution, Hospital 2 and 3 used different imaging parameters for the aortoiliac station, upper leg and lower leg station. 
Change in QoL was assessed by the rating scale (RS) (12) "wo generic questionnaires (Short Form 36 (SF-36) $(13,14)$ and Euroqol-5D (15)) and a diseasespecific questionnaire (VascuQol (16)), which were completed (by the patients themselves) at baseline, 2 weeks, 3 months and 6 months after imaging workup. Patients returned their questionnaires by mail. We selected the following four most responsive health dimensions of the SF-36 for patients with PAD: physical functioning, role physical functioning, bodily pain and general health $(14,16,17)$.

\section{Costs}

Costs calculations were performed from a hospital perspective which means that all costs made inside the hospital were estimated, and according to the Dutch guidelines for cost calculations in health care (18). To calculate the mean total cost per imaging strategy per patient, we collected all diagnostic costs, outpatient visits costs, the physician costs and therapeutic costs. Costs were collected at each hospital during 6 months after the date of the initial imaging workup. All costs were reported in euros for the year 2002 (the exchange rate was 0.90 euro per US dollar, Jan, 2002).

Diagnostic costs consisted of the costs of initial and additional vascular imaging, and were computed by adding the directly and non-directly assignable costs.

Directly assignable costs contained equipment costs (such as investment), dedicated MR coils, maintenance, room construction costs, personnel costs, and material costs. The equipment costs were computed by adding the annualized costs of radiological equipment and the annual equipment maintenance costs, both divided by the proportion of the total available room time ( $80 \%$ of a 40 hour work-week) and with an annual $3 \%$ discount rate (18-20). Personnel costs were based on multiplying the mean wages (including the social security of $37 \%$ ) with the measured time spent on an imaging test for each personnel-category. Material costs were computed by adding cost prices of the materials used during imaging such as contrast agent, syringes and/or interventional catheters.

Non-directly assignable costs comprised housing costs, costs of supporting departments $_{\mathrm{r}}$ and overhead costs. The housing costs for the radiological rooms were based on the housing costs per $\mathrm{m}^{2}$ per year multiplied with the room surface. Costs of supporting departments was based on the records of Financial and Economics Department of each hospital. The overhead costs were estimated to be $15 \%$ of directly assignable costs (18). Therapeutic costs included costs for percutaneous vascular interventions (i.e. percutaneous angioplasty, stent placement, and thrombolysis), vascular surgery (i.e. bypass surgery, endarteriectomy, and amputation), and costs associated hospital admissions or due to complications. Costs of vascular surgery were measured and calculated in a similar fashion as the diagnostic costs using the data of another comparable study (21). The costs of the number of outpatient visits, days of hospital and / or intensive care admissions were based on the national estimates (18). 


\section{Statistical Analysis}

The results were analyzed according to the intention-to-(diagnose)-and-treat principle and the CONSORT guidelines $(22,23)$. As a result, patients who died during follow-up were not excluded from cost analysis and the worst possible scores were used to represent $Q$ oL at 6 months follow-up. For all other patients, data that was missing due to questionnaires that were not retumed had to be excluded from the analysis of change in QoL. Missing items on returned questionnaires were imputed using the mean value of that variable.

The sample size calculation was based on QoL, because to our opinion it is of major importance not to miss a clinically relevant difference in this outcome measure. It was expected that after treatment $40-50 \%$ of the patients had a considerable improvement of their symptoms as detected by the dimensions pain and physical functioning of the SF-36 (14). In order to detect a clinically important difference of $15 \%$ with $80 \%$ power and a 2 -tailed a level of .05 , we calculated a final target sample of 340 patients. Anticipating a $5 \%$ drop out rate our recruitment goal was 357 patients.

Differences in costs between DUS and CE-MRA were adjusted for the baseline characteristics that were assumed to potentially influence the outcomes according to the Trans Atlantic Inter-Society Consensus (TASC) (24). Therefore, the following determinants at baseline that were recommended by the TASC were entered (at once) in a multivariable linear regression analysis: the presence of renal diseases (i.e. renal transplantation and renal insufficiency), cardiac diseases, cerebrovascular diseases, or diabetes mellitus, disease severity (critical ischemia vs. claudication), hospital setting, and study group.

The Student $t$ test was used to test differences between the other outcome measures of both imaging workup strategies. Since multiple statistical tests were performed a $P$-value $\leq .01$ was considered to be statistically significant. All analyses were performed using SPSS version 11.0 (SPSS, Inc., Chicago, II).

\section{Results}

A total of 720 patients were evaluated for trial eligibility, of whom 363 patients were excluded for different reasons. Figure 1 shows more details on the flow of patients through the trial. After randomization, 180 patients were allocated to CE-MRA and 177 patients were allocated to DUS. Two patients in the CE-MRA group and three patients in the DUS group either died or withdrew from the study prior to undergoing imaging, and were therefore excluded from analysis. Consequently, the cost analysis was performed on 178 patients in the CE-MRA group and 174 patients in the DUS group. During follow-up 4 other patients died in the CE-MRA and 5 patients died in the DUS group. 
Figure 1 Study Flow Chart

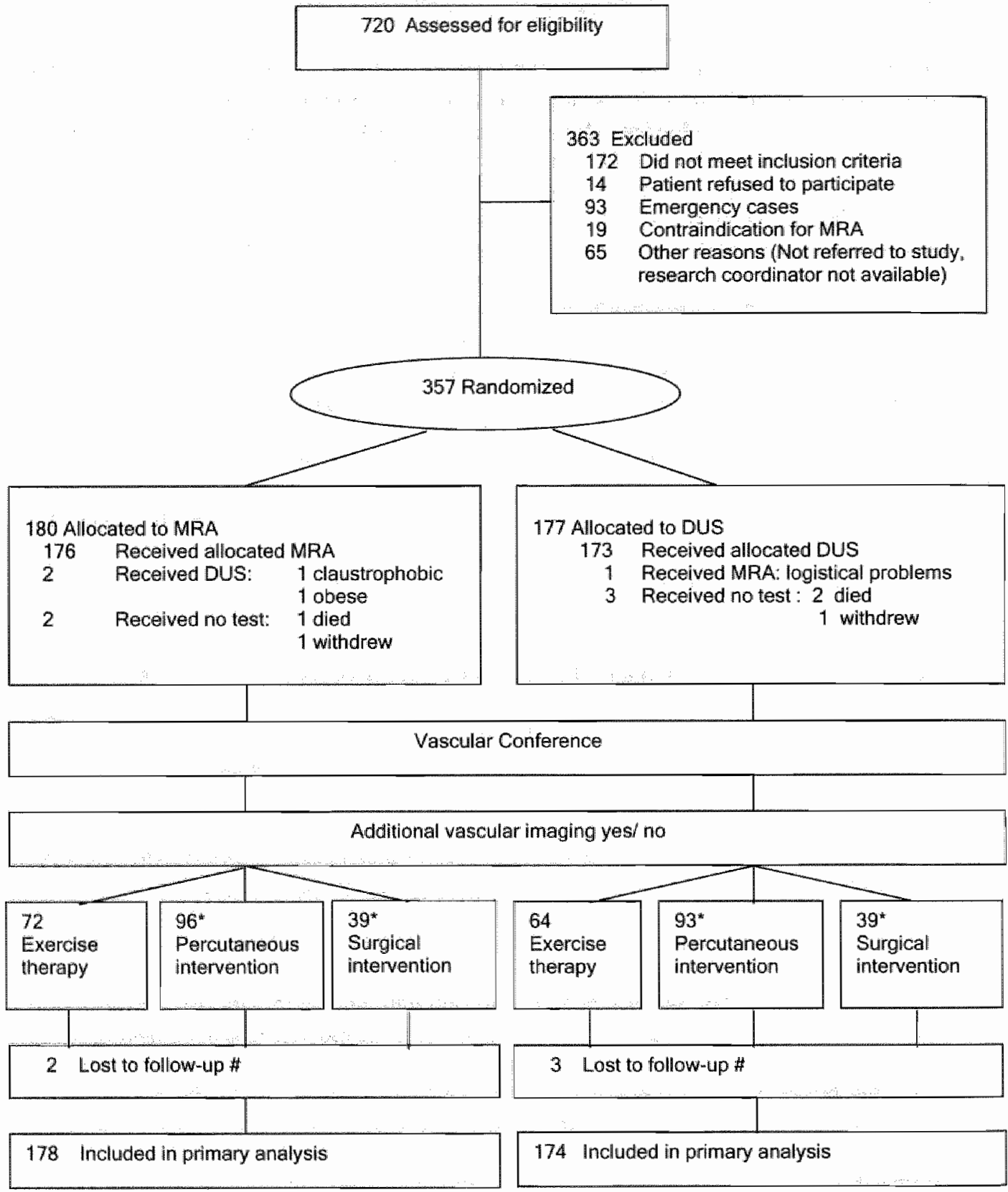

* Number of interventional procedures, (some patients underwent several interventional procedures).

\# Lost to follow-up were patients that received no imaging test. 
The distribution of baseline characteristics was similar in the CE-MRA and DUS group (Table 2), except for co-morbidity that occurred slightly more often in the CEMRA group. The distribution of the severity of PAD expressed as Rutherford classification was equal for both groups. The CE-MRA group included 19 patients (11\%) and DUS group included 20 patients (11\%) with critical ischemia.

Table 2

Baseline Characteristics

\begin{tabular}{|c|c|c|c|c|}
\hline$\ldots$ & \multicolumn{2}{|c|}{ CE-MRA } & \multicolumn{2}{|l|}{ DUS } \\
\hline Total number of patients $(\mathrm{N})$ & 178 & & 174 & \\
\hline \multicolumn{5}{|l|}{ Number of patients at each hospital } \\
\hline Eindhoven & 49 & & 50 & \\
\hline Maastricht & 92 & & 87 & \\
\hline Nijmegen & 37 & & 37 & \\
\hline Male / female & 117 & $(66 \%) / 61(34 \%)$ & 122 & $(70 \%) / 52(30 \%)$ \\
\hline Age years (mean, (SD)) & 65 & (11) & 65 & $(\| 1)$ \\
\hline \multicolumn{5}{|l|}{ Medical history } \\
\hline Tobacco use (ever/ never) & 167 & $(94 \%) / 11(6 \%)$ & 160 & $(92 \%) 14(8 \%)$ \\
\hline Diabetes mellitus & 41 & $(23 \%)$ & 30 & $(17 \%)$ \\
\hline Hypertension & 88 & $(49 \%)$ & 79 & $(45 \%)$ \\
\hline Hyperlipidemia & $83^{*}$ & $(51 \%)$ & $77^{*}$ & $(49 \%)$ \\
\hline Renal faillure & $16^{*}$ & $(9 \%)$ & $10^{i k}$ & $(6 \%)$ \\
\hline Cardiac disease & 72 & $(40 \%)$ & $63^{*}$ & $(36 \%)$ \\
\hline Cerebrowascular disease & 27 & $(15 \%)$ & 27 & $(16 \%)$ \\
\hline Previous revascularisation & 67 & $(38 \%)$ & 60 & $(35 \%)$ \\
\hline \multicolumn{5}{|l|}{ Rutherford classification } \\
\hline Mild- severe (Rutherford 1-4) & 159 & $(89 \%)$ & 154 & $(89 \%)$ \\
\hline Ischemia-ulcers (Rutherford 5-7) & 19 & $(11 \%)$ & 20 & $(11 \%)$ \\
\hline \multicolumn{5}{|l|}{ Exercise data at baseline (mean, (SE)) } \\
\hline $\mathrm{ABPI}$ at rest & 0.60 & $(0.01)$ & 0.60 & $(0.02)$ \\
\hline ABPI after exercise & 0.40 & $(0.02)$ & 0.37 & $(0.02)$ \\
\hline Waiking distance (meter) & 178 & (8) & 189 & (8) \\
\hline \multicolumn{5}{|l|}{ Quality-of-life (mean) } \\
\hline Rating Scale (RS) & 56 & & 60 & \\
\hline SF-36 physical functioning & 38 & & 40 & \\
\hline SF-36 role physical & 34 & & 35 & \\
\hline SF-36 pain & 44 & & 45 & \\
\hline SF-36 general heatth & 49 & & 52 & \\
\hline Eurogol-5D & 0.52 & & 0.55 & \\
\hline VascuQol total & 3.7 & & 3.7 & \\
\hline
\end{tabular}

Data are numbers of patients and percentages in parentheses. "For some patients data on baseline characteristics were not available: The percentages are calculated based on the number of patients for whom data was awailable. 


\section{Outcome Measures}

Both CE-MRA and DUS were associated with high therapeutic confidence scores for making a treatment decision after the imaging workup. The mean therapeutic confidence score was 8.2 for the CE-MRA group and 7.5 for the DUS group, which was significantly higher for the CE-MRA group $(P<.001)$.

Table $3 \quad$ Change in Outcome Mleasurements after Follow-up

\begin{tabular}{|c|c|c|c|c|c|c|}
\hline \multirow[t]{2}{*}{ (A) Mean change } & \multicolumn{2}{|l|}{2 Weeks } & \multicolumn{2}{|l|}{3 Months } & \multicolumn{2}{|l|}{6 Months } \\
\hline & CE-MRA & DUS & CE-MRA & DUS & CE-MRA & DUS \\
\hline \multicolumn{7}{|l|}{$\triangle 1$ Disease severity } \\
\hline$\Delta$ Rutherford category & & & & & +1 & +1 \\
\hline$\Delta$ Walking distance $(m)$ & & & & & 25 & 23 \\
\hline$\triangle A B P \|$ at rest & & & & & 0.15 & 0.14 \\
\hline$\triangle \mathrm{ABPl}$ after exercise & & & & & 0.22 & 0.25 \\
\hline \multicolumn{7}{|l|}{$\triangle$ Quality-of-life } \\
\hline$\triangle$ Rating Scale & 2 & -2 & 3 & 1 & 3 & 1 \\
\hline$\Delta S F-36$ physical funct \# & -2 & 2 & 9 & 8 & 10 & 10 \\
\hline$\Delta S F-36$ role physical & 0 & 1 & 7 & 8 & 6 & 13 \\
\hline$\Delta S F-36$ bodily pain & -1 & 1 & 9 & 9 & 8 & 9 \\
\hline$\Delta S F-36$ general health & -1 & -1 & -1 & -2 & -3 & -2 \\
\hline$\Delta$ Eurogol-5D & -0.04 & -0.01 & 0.04 & 0.03 & 0.05 & 0.03 \\
\hline$\triangle$ VascuQol total & 0.0 & 0.1 & 0.7 & 0.7 & 0.8 & 0.9 \\
\hline
\end{tabular}

$\Delta=$ Changes in indicators for disease severity and QoL were calculated by subtracting the baseline score from the score measured at 6 months after the date of the diagnostic exam. Change in disease severity was only determined after 6 months. SF-36 physical funct $=$ SF-36 physical functioning.

With respect to changes in Rutherford classification, maximum treadmill walking distance, $\mathrm{ABPI}$ at rest and $\mathrm{ABPI}$ after exercise, there was no statistically significant difference between both groups with respect to improvement of disease severity (Table 3).

The response rates for the QoL questionnaires were $98 \%$ at baseline, $95 \%$ at 2 weeks, $91 \%$ at 3 months, and $88 \%$ after 6 months follow-up. Moreover, all returned questionnaires had high completion rates for the individual dimensions (completion rates varied from $97.2 \%$ to $99.7 \%$ ).

Table 3 shows no statistically significant differences in improvement of QoL after 2 weeks, 3 and 6 months between CE-MRA and DUS. 
Table $4 \quad$ Number of Imaging Exams and Interventions and Distribution of Costs

\begin{tabular}{|c|c|c|c|c|c|c|}
\hline \multirow{3}{*}{ Costs (in euros per patient) } & \multirow{2}{*}{\multicolumn{2}{|c|}{$\begin{array}{l}\text { Frequency } \\
\text { CE-MRA DUS } \\
\end{array}$}} & \multicolumn{4}{|c|}{ Costs mean ( 5 and $95 \%$ percentile) } \\
\hline & & & \multicolumn{2}{|c|}{ CE-MRA $(N=178)$} & \multicolumn{2}{|c|}{ DUS $(N=174)$} \\
\hline & $180 \$$ & 174 & 473 & $(305-599)$ & 405 & $(40-168)$ \\
\hline Additional imaging before therapy \# & 19 & 47 & 46 & $(0-105)$ & 207 & $(0-1121)$ \\
\hline Additional imaging after therapy & 21 & 22 & 28 & $(0-96)$ & 46 & $(0-125)$ \\
\hline Total additional imaging \# & 40 & 69 & 118 & $(0-728)$ & 323 & $(0-1766)$ \\
\hline $\begin{array}{l}\text { Total diagnostic costs } \\
\text { (including initial test) }\end{array}$ & & & 532 & $(308-960)$ & 356 & $(57-1434)$ \\
\hline Outpatient visits & 516 & 527 & 182 & $(80-280)$ & 189 & $(80-350)$ \\
\hline Percutaneous interventions & 96 & 93 & 1193 & $(0-354 \| 1)$ & 1131 & $(0-3252)$ \\
\hline Surgical procedures & 39 & 39 & 1105 & $(0-6808)$ & 1037 & $(0-5207)$ \\
\hline Total costs & & & 3012 & $(499-10474)$ & 2713 & $(180-7769)$ \\
\hline
\end{tabular}

$N=$ Number of patients, $\ddagger$ Two extra CE-MRA exams were necessary (in1 patient due to reconstruction failure of the CE-MRA dataset and in 1 patient the aorta was not depicted). \# The casts of the initial test are not included. "Adjusted for small imbalances in disease severity (critical ischemia vs claudication), renal disease, cerebrovascular disease, cardiac disease, diabetes mellitus at baseline, and for hospital. † Positive cost difference indicates that MRA is more expensive than DUS and negative cost difference indicates that DUS is more expensive than CE-MRA.

Table 4 shows that before therapy, 19 additional vascular imaging exams were performed in the CE MRA group, whereas in the DUS group 47 additional vascullar imaging exams were performed. During 6 months follow-up, in total 40 additional vascular imaging exams were performed in the CE-MRA group compared to 69 additional vascular imaging exams in the DUS group. Thus, the total number of additional vascular imaging exams was reduced by $42 \%$ in the CE-MRA group ( $R$ .001 ). For the DUS group, these additional exams consisted mainly of DSA and CEMRA exams. The total number of additional diagnostic DSA exams was reduced by $63 \%$ in the CE-MRA compared to the DUS group (11 versus 30 DSA exams, $P=$ .001). In the DUS group 20 additional CE-MRA exams were performed compared to 1 additional CE-MRA exam in the CE-MRA group. 
Table 4

Continued

\begin{tabular}{|c|c|c|c|}
\hline \multirow{3}{*}{$\begin{array}{l}\text { Costs (in euros per patient) } \\
\text { Initiall test }\end{array}$} & \multicolumn{3}{|c|}{ Adjusted difference $(95 \% \mathrm{Cl})$} \\
\hline & \multicolumn{2}{|c|}{ CE-MRA versus DUS t } & \multirow{2}{*}{$\frac{\text { Pvalue }}{<.001}$} \\
\hline & 349 & $(334$ to 364$)$ & \\
\hline Additional imaging before therapy \# & -167 & $(-246$ to -89$)$ & $<.001$ \\
\hline Additional imaging after therapy & -15 & $(-54$ to 24$)$ & .45 \\
\hline Total additional imaging \# & -202 & $(-314$ to -91$)$ & $<.001$ \\
\hline $\begin{array}{l}\text { Total diagnostic costs } \\
\text { (including initial test) }\end{array}$ & 167 & $(79$ to 255$)$ & $<.001$ \\
\hline Outpatient visits & -8 & $(-23$ to 7$)$ & .30 \\
\hline Percutaneous interventions & 91 & $(-214$ to 407$)$ & .54 \\
\hline Surgical procedures & 16 & $(-540$ to 572$)$ & .95 \\
\hline Total costs & 272 & $(-377$ to 921$)$ & .41 \\
\hline
\end{tabular}

The costs are calculated in euros for the year 2002 (the exchange rate was 0.90 euro per US dallar, Jan 2002).

\section{Costs}

Overall, the mean total costs per patient, consisting of all diagnostic, therapeutic and outpatient visits costs, were $€ 272$ higher in the CE-MRA group than in the DUS group (Table 4). This difference was not significant $(95 \% \mathrm{Cl}, € 377$ to $€ 921 ; P=$ 41).

The mean unit cost for the initial test per patient was $€ 105$ for a DUS exam and $€ 473$ for a CE-MRA exam. The mean costs for total additional imaging per patient during 6 months follow-up was $€ 118$ in the CE-MRA group compared to $€$ 323 in the DUS group (Table 4). The total additional imaging costs were significantly lower in the CE-MRA group compared to the DUS group (mean difference was $€ 202$ per patient, $P<.001$ ). Although, the CE-MRA was associated with lower total additional imaging costs, the total diagnostic costs of the CE-MRA were $€ 167$ higher compared to DUS $(P<.001)$. One-way sensitivity analysis showed that the higher total diagnostic costs for the CE-MRA were explained by the higher costs of the initial imaging test in the CE-MRA group, due to the high investment costs of the MR scanner. In the one-way sensitivity analysis, the investment costs of the MR scanner were reduced by $50 \%$, which resulted in a non-statistically significant difference of total diagnostic costs between the CE-MRA and DUS (adjusted difference $€ 52$; $95 \% \mathrm{Cl}-€ 33$ to $€ 138 ; P=.23$ ).

Furthermore, Table 4 shows that the costs for outpatient visits were not significantly different between the CE-MRA and DUS groups $(P=.30)$. The number and costs for revascularisation through percutaneous transluminal angioplasty (PTA) and surgical procedures were not significantly different between both groups $(P>.05)$.

A subgroup analysis showed no statistically significant differences in total additional imaging costs, total diagnostic costs and total costs between DUS and CEMRA between the three hospitals. 


\section{Discussion}

CE-MRA and DUS performed equally well in the initial imaging workup for patients with $\mathrm{PAD}$, albeit that the two imaging modalities had different qualities that counted in their favor. CE-MRA reduced the number of additional vascular imaging tests and improved the therapeutic confidence score. On the other hand DUS was associated with significantly lower total diagnostic costs compared to CE-MRA due to the high investment costs of the MR scianner. The total costs (i.e. all diagnostic, therapeutic and outpatients visits costs as well as improvement in QoL and disease severity after 6 months) were comparable for the CE-MRA and the DUS group.

Ultimately the choice between DUS and CE-MRA for the initial imaging workup for patients with PAD will depend on local expertise with these techniques and cost considerations relevant to the particular setting. DUS has very good results in terms of sensitivity and specificity even regarding depiction of the lower legs, provided that the ultrasonographers performing the exam are highly experienced (2527). Similarly, the image quality of MRA depends on the quality of the equipment and experience of the MR technologists. For example, high spatial resolution is a prerequisite for optimal depiction of the vascular tree, and high temporal resolution is important to avoid projection of disturbing veins over the arteries. As a result, dedicated MR receiver coils, up-to-date imaging protocols and the use of contrast agents are necessary to obtain optimal image quality. Studies have shown that MRA without the use of contrast agents (e.g. Using time of flight sequences) reduced diagnostic accuracy compared to MRA using contrast agents $(28,29)$.

A major advantage of DUS is that the total diagnostic costs are lower compared to CE-MRA. The higher total diagnostic costs of CE-MRA are explained by the higher costs of the initial CE-MRA exam, particularly due to high investment costs of the MR scanner. It should be noted, however, that MRA is a relatively new imaging technique, which may in part explain the high investment costs of the MR scanner. One may expect that in the future these costs decrease as MR scanners become more widely available. As was shown in this study "with a $50 \%$ reduction in the investment costs of the MR scanner, the total diagnostic costs were almost equal for CE-MRA and DUS. Furthermore, although the difference in diagnostic costs was statistically significant, the magnitude of the difference was not that large and a department may consider the additional expense justified.

Using CE-MRA resulted in a lower need for additional vascular imaging, particularly diagnostic DSA exams than for DUS. This finding was in accordance with the findings of Leiner et al. who showed that surgeons who had to define treatment plans were less likely to order diagnostic DSA after CE-MRA than after DUS (8). In our study the reduction in additional vascular imaging exams is consistent with the significantly higher therapeutic confidence score after CE-MRA than after DUS. The higher therapeutic confidence of the surgeons after CE-MRA indicates that treatment planning was more efficient after CE-MRA than after DUS. An explanation is that CEMRA presents the arteries as a roadmap, which allows better visualization of the location, severity and extent of the stenosis and quality of the outflow arteries.

Although treatment planning was more efficient after CE-MRA, neither CEMRA nor DUS seemed to influence the therapeutic decision, as is suggested by the 
fact that the number and nature of interventional procedures as well as therapeutic costs were similar for both groups. The foremost contributors of the total costs were the costs of therapy and possible complications after therapy (30). Our study also showed that despite the higher total diagnostic costs in the CE-MRA group, similar therapeutic costs resulted in símilar total costs for both DUS and CE-MRA groups.

Our study had several limitations. It is expected that the CE-MRA strategy might be more cost-effective in patients with critical ischemia. These patients often undergo distal bypass surgery and require more adequate depiction of the lower leg arteries, which may be better obtained by CE-MRA. However in our study, only 39 $(11 \%)$ of all 352 patients had critical ischemia, and consequently this group was too small to allow a separate subgroup analysis.

Another study limitation was that the computation of costs was performed using a hospital perspective, instead of using a societal perspective as frequently recommended. $(22,23)$. Calculating costs according to the societal perspective requires adding all costs inside and outside the healthcare sector, such as direct costs (patient costs) and indirect costs (costs due to production losses). We chose for a hospital perspective, because previous cost analyses concerning the management of patients with PAD showed that patient costs were relatively low in both Dutch and United States settings $(31,32)$. Furthermore, the majority of the patients with PAD were retired from the workforce, therefore the costs due to production losses will be negligible (33).

Several small imbalances in the distribution of baseline characteristics between the CE-MRA and the DUS group were observed in spite of randomization. For example, comorbidity occurred somewhat more frequently in the CE-MRA group than in the DUS group. Although the differences were small, we adjusted for relevant baseline characteristics in a multivariable linear regression analysis. In addition, we adjusted for hospital setting.

In conclusion, the choice between DUS and CE-MRA for the initial imaging workup for patients with PAD depends on the local expertise with these techniques and cost considerations relevant to the local setting. Centers that seek to improve their imaging work-ups might opt to expand their expertise in CE-MRA rather than DUS, since CE-MRA increases the physician's confidence in taking treatment decisions, which reduces the need for additional imaging. 


\section{References}

1. Meijer WT, Hoes AW, Rutgers D, Bots ML, Hofman A, Grobbee DE. Peripheral arterial disease in the elderly: The Rotterdam Study. Arterioscler Thromb Vasc Biol 1998;18(2):185-192.

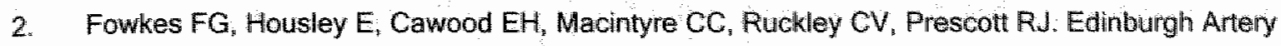
Study: prevalence of asymptomatic and symptomatic peripheral arterial disease in the general population. Int J Epidemiol 1991;20(2):384-392.

3. Elsman $\mathrm{BH}_{4}$ Legemate DA, van der Heyden $\mathrm{FW}$, de Vos $\mathrm{H}$, Mali WP, Eikelboom $\mathrm{BC}$. The use of color-coded duplex scanning in the selection of patients with lower extremity arterial disease for percutaneous transiuminal angioplasty: a prospective study. Cardiovasc Intenvent Radiol 1996;19(5):313-316.

4. Elsman BH, Legemate DA, van der Heijden FH, de Vos HJ, Mali WP, Eikelboom BC. Impact of ultrasonographic duplex scanning on therapeutic decision making in lower-limb arterial disease. Br J Surg 1995;82(5):630-633.

5. Koelemay MJ, den Hartog D, Prins MH, Kromhout JG, Legemate DA, Jacobs MJ. Diagnosis of arteriall disease of the lower extremities with duplex ultrasonography. Br J Surg 1996;83(3):404409.

6. de Vries SO, Hunink MG, Polak JF. Summary receiver operating characteristic curves as a technique for meta-analysis of the diagnostic performance of duplex ultrasonography in peripheral arterial disease. Acad Radiol 1996;3(4):361-369.

7. Visser $\mathrm{K}$, Hunink MG. Peripheral arterial disease: gadolinium-enhanced MR angiography versus collor-guided duplex US--a meta-analysis. Radiology 2000;216(1):67-77.

8. Leiner $T_{*}$, Tordoir $\mathrm{JH}_{3}$ Kessels $\mathrm{AG}_{*}$ et al. Comparison of treatment plans for peripheral arterial disease made with multi-station contrast medium-enhanced magnetic resonance angiography and duplex ultrasound scanning. J Vasc Surg 2003;37(6):1255-1262.

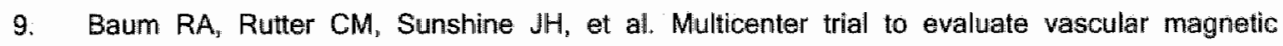
resonance angiography of the lower extremity. American College of Radiology Rapid Technology Assessment Group. JAMA. 1995;274(11):875-880.

10. Carpenter $\mathrm{JP}_{1}$ Baum RA, Holland GA, Barker CF. Peripheral vascular surgery with magnetic resonance angiography as the sole preoperative imaging modality. J Vasc Surg 1994;20(6):861869; discussion 869-871.

11. Carpenter JP, Golden MA, Barker CF, Holland GA, Baum RA. The fate of bypass grafts to angiographically occult runoff vessels detected by magnetic resonance angiography. $\mathrm{J}$ Vasc Surg 1996;23(3):483-489.

12. Bosch $\mathrm{JL}_{\|}$Hunink MG. The relationship between descriptive and valuational quality-of-life measures in patients with intermittent claudication. Med Decis Making 1996; 16(3):217-225.

13. McHorney CA, Ware JE, Jr., Lu JF, Sherbourne CD. The MOS 36-item Short-Form Health Survey (SF-36): III. Tests of data quality "scaling assumptions, and reliability across diverse patient groups. Med Care 1994;32(1):40-66.

14. Bosch JL, van der Graaf $Y$, Hunink MG. Health-related quality of life after angloplasty and stent placement in patients with iliac artery occlusive disease: results of a randomized controlled clinicall trial. The Dutch Illiac Stent Trial Study Group. Circulation 1999;99(24):3155-3160.

15. Dolan P. Modeling valuations for EuroQol health states. Med Care 1997;35(11):1095-1108.

16. Morgan MB, Crayford T, Murrin B, Fraser SC. Developing the Vascular Quality of Life Questionnaire: a new disease-specific quality of life measure for use in lower limb ischemia. $J$ Vasc Surg 2001;33(4):679-687. 
17. Currie IC, Wilson YG, Baird RN, Lamont PM. Treatment of intermittent claudication: the impact on quality of life. Eur J Vase Endovase Surg 1995;10(3):356-361.

18. Oostenbrink $J B$, Koopmanschap MA, Rutten FF. Standardisation of costs: the Dutch Manulal for Costing in economic evaluations. Pharmacoeconomics 2002;20(7):443-454.

19. Hunink MG, Glasziou PP. Decion making in health and medicine-integrating evidence and values. Cambridge: Cambridge University Press; 2001.

20. Gold M. Panel on cost-effectiveness in health and medicine Med Care 1996;34(12 Suppl):DS197-199.

21. Visser $K_{\text {; }}$ de Vries SO, Kitslaar PJ, van Engellshoven JM, Hunink MG. Cost-effectiveness of diagnostic imaging work-up and treatment for patients with intermittent claudication in The Netherlands. Eur J Vasc Endovasc Surg 2003;25(3):213-223.

22. Siegel JE, Weinstein MC, Russell LB, Gold MR. Recommendations for reporting costeffectiveness analyses. Panel on Cost-Effectiveness in Health and Medicine. JAMA $1996 ; 276(16) ; 1339-1341$.

23. Moher $D_{n}$ Schulz KF, Altman DG. The CONSORT statement: revised recommendations for improving the quality of reports of parallel-group randomised trials. Clin Oral Investig 2003;7(1):2-7.

24. Dormandy JA, Rutherford RB. Management of peripheral arterial disease (PAD). TASC Working Group. TransAtlantic Inter-Society Concensus (TASC). J Vasc Surg 2000;31(1 Pt 2):S1-S296.

25. Koelemay MJ, Legemate DA, van Gurp J, Ponson AE, Reekers JA, Jacobs MJ. Colour duplex scanning and pulse-generated run-off for assessment of popliteal and cruropedal arteries before peripheral bypass surgery. Br J Surg 1997;84(8):1115-1119.

26. Koelemay MJ, Legemate DA, de Vos $H$, et al. Duplex scanning allows selective use of arteriography in the management of patients with severe lower leg arterial disease. J Vasc Surg 2001;34(4):661-667.

27. Grassbaugh $J A_{1}$ Nelson PR, Rzucidlo EM, et al. Blinded comparison of preoperative duplex ultrasound scanning and contrast arteriography for planning revascularization at the level of the tibia. J Vasc Surg 2003;37(6):1186-1190.

28. Swan JS, Kennell TW, Acher $\mathrm{CW}_{n}$ et al. Magnetic resonance angiography of aorto-iliac disease. Am J Surg, 2000; 180(1):6-12.

29. Eiberg JP, Lundorf $E$, Thomsen $C$. Sichroeder TV. Peripheral vascular surgery and magnetic resonance arteriography-a review. Eur J Vasc Endovasc Surg 2001;22(5):396-402.

30. Jansen $R$, de Vries $S_{i}$ Cullen $K$, Donaldson $M$, Hunink $M$. Cost-identification analysis of revascularization procedures on patients with peripheral arterial occlusive disease. Journal of Vascular Surgery $1998 ; 28(4): 617-623$.

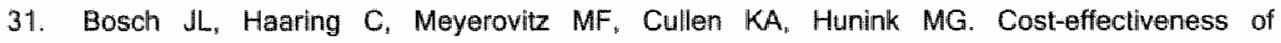
percutaneous treatment of iliac artery occlusive disease in the United States. AJR Am J Roentgenol 2000; 175(2):517-521.

32. Bosch JL, Tetteroo E, Mali WP, Hunink MG. lliac arterial acclusive disease: cost-effectiveness analysis of stent placement versus percutaneous transluminal angioplasty. Dutch lliac Stent Trial Study Group. Radiology 1998;208(3):641-648.

33. Koopmanschap MA, Rutten FF, wan Ineveld BM, van Roijen $L$. The friction cost method for measuring indirect costs of disease. J Health Econ 1995; 14(2):171-189. 


\section{CHAPTER 5}

\section{Multicenter Randomized Controlled Trial of the Costs and Effects of Non-Invasive Diagnostic Imaging in Patients with Peripheral Disease: The DIPAD Trial}

Submitted

Rody Ouwendijk, Marianne de Vries, Theo Stijnen, Peter M. T. Pattynama, Marc R.H.M. van Sambeek, Jaap Buth, Alexander V. Tielbeek, Daan A. van der Vliet, Leo J. SchutzeKool, Peter J.E.H.M. Kitslaar, Michiel W. de Haan, Jos M.A. van Engelshoven,M. G. Myriam Hunink.

This study was supported by a grant (nr. 945-01-039) from ZonMw, Netherlands Organisation for Health Research and Development, The Hague, The Netherlands 


\section{Abstract \\ Purpose}

Non-invasive diagnostic imaging is increasingly used for the evaluation of peripheral arterial disease. The purpose of this study was to compare the costs and effects of three non-invasive imaging tests as the initial imaging test in the diagnostic workup of patients with peripheral arterial disease.

\section{Materials and Methods}

Of 984 patients assessed for eligibility, 514 patients with peripheral arterial disease were randomized to magnetic resonance angiography (MRA) or duplex ultrasound (DUS) in three hospitals and to MRA or computed tomographic angiography (CTA) in one hospital. The outcome measures included the clinical utility, functional patient outcomes, quality of life, and actual diagnostic and therapeutic costs related to the initial imaging test during 6 months follow-up. Clinical utility was assessed with therapeutic confidence $(0-10)$ in the initial imaging test and the number of additional vascular imaging tests performed. Patient outcomes included change in anklebrachial index, maximum walking distance, and clinical status.

\section{Results}

With adjustment for potentially predictive baseline variables, the learning curve, and hospital setting, a significant higher confidence and less additional imaging were found for MRA and CTA compared to DUS. There were no statistically significant differences in improvement in functional patient outcomes and quality of life between the groups. The total costs were significantly higher for MRA and DUS compared to CTA.

\section{Conclusions}

The results suggest that both CTA and MRA are clinically more useful than DUS and that CTA leads to cost-savings compared to both MRA and DUS in the initial imaging evaluation of peripheral arterial disease. 


\section{Introduction}

Peripheral arterial disease (PAD) is the expression of atherosclerosis in the lower limb distal to the aortic bifurcation, which is a major problem in the population of 55 years and older (1). The first manifestation of symptomatic PAD is usually intermittent claudication. In a minority of patients, the disease progresses to critical limb ischemia, i.e. rest pain and tissue necrosis. If PAD is suspected on the basis of patient history and physical examination, ankle-brachial indices (ABD) are generally measured to document the severity of the disease.

Diagnostic imaging is performed when $\mathrm{PAD}$ becomes lifestyle limiting and $a$ revascularization procedure is considered. Non-invasive imaging tests including Duplex ultrasound (DUS), computed tomographic angiography (CTA), and magnetic resonance angiography (MRA) are increasingly used for the initial evaluation of patients with PAD. DUS provides both anatomical and functional information about the arterial system and has been shown to be a reliable modality with fairly good sensitivity and specificity $(2,3)$. DUS is, however; operator dependent and does not provide a precise roadmap for planning treatment. Both MRA and CTA are relatively new non-invasive vascular imaging tests used in the diagnostic workup of peripheral arterial disease. Both modalities provide three-dimensional images of the arterial system with high sensitivity and specificity (4-11). Disadvantages of MRA include the higher investment cost for equipment, the small number of cases in whom the image is uninterpretable due to artifacts, and the fact that some patients are claustrofobic or have a contraindication for MR scanning. The main disadvantages of CTA are the use of radiation, the use of potentially nephrotoxic iodinated contrast media, vessel wall calcifications that affect image interpretation, and the time-consuming $3 \mathrm{D}$ reconstruction techniques. The question arises which imaging test is preferred in the diagnostic work-up of PAD.

To determine which non-invasive test is preferred as initial imaging test in clinical practise we need to take into account not only the diagnostic accuracy of each test, but also the related effects of diagnostic imaging tests on treatment planning, functional improvement, quality of life, and costs $(12,13)$. For this purpose we designed the Diagnostic Imaging of Peripheral Arterial Disease (DIPAD) randomized trial to compare outcomes following DUS, MRA, and CTA as the initial ilmaging test in the diagnostic workup of patients with peripheral arterial disease. Primary outcomes evaluated were quality of life and costs. Secondary outcomes evaluated were clinical utility and functional patient outcomes.

\section{Material and Methods}

\section{Study Patients}

Between December 2001 and September 2003, the DIPAD trial consecutively enrolled 514 patients at 4 Dutch hospitals. Men and women at least 18 years old with symptomatic PAD who were referred from the Department of Vascular Surgery for diagnostic imaging workup to evaluate the feasibility of a revascularization procedure 
were eligible for enrollment. PAD was defined as symptoms of intermittent claudication and/ or critical ischemia with an $A B \mid \leq 0.90(14,15)$.

Patients were excluded if they had contraindications for MRA (eg, pacemaker, cerebral vessel clipping, or claustrophobia) or CTA (eg, severe renal insuffiency or adverse reactions to iodinated contrast agent), or if they needed an acute intervention at the time of randomization.

\section{Study Design}

This was an empirically based and pragmatic multicenter randomized controlled trial evaluating the costs and effects of non-invasive diagnostic imaging in patients with $\mathrm{PAD}$. That is, we designed the trial to reflect clinical practice as it can be implemented rather than creating a strictly controlled "but probably unrealistic, experimental setting (13). The study protocol was approved by the hospital institutional review board for all participating centers, and informed consent was obtained from all patients. The study was performed following Good Clinical Practice guidelines (16). Data were analyzed and reported in accordance with the CONSORT guidelines (17). Patients meeting all eligibility criteria were randomly assigned to undergo MRA or the currently employed test, which was DUS in three hospitals and CTA in one hospital as the initial imaging test. Randomization was performed centrally and took place through the Trial Coordinating Center by telephone. A computer generated list for the strategy assignment was used. Eligible patients were enrolled by one of several researchers who were all unaware of the randomization sequence. Following randomization, patients and clinicians were not blinded for the imaging strategy because this would have been highly impractical and inconsistent with our pragmatic study design.

\section{Imaging Techniques and Evaluation}

DUS was performed by qualified, experienced vascular technologists with 5 and 7.5 $\mathrm{MHz}$ transducers. On the basis of patient history and findings at physical examination, the referring vascular surgeons determined the extent of the DUS examination (aortoiliac, femoropopliteal, crural). The hemodynamic significance of lesions was graded by peak systolic velocity (PSV) ratios, calculated as the PSV in the stenosis divided by the PSV in the prestenotic or poststenotic region. The technologists graded stenosis on a five-point ordinal scale and recorded the findings on a standardized reporting sheet.

All MR examinations were performed on a 1.5-T imager. A body coil or a dedicated peripheral vascular phased-array coill was used for signal reception. In 3 hospitals the protocol included a bolus-chase MRA with single biphasic contrast material injection, automated table movement, and real time bolus monitoring. In one hospital a multi-injection protocol was used. In all hospitals a subtraction technique was used before maximum intensity projections (MIP) were generated.

CTA was performed on a 16-slice multi-detector scanner. A bolus-tracking technique was used with automated table movement and automated bolus detection. 
Before generating MIPs, a segmentation technique was used to remove bony structures and vessel wall calcifications.

Radiologists with extensive experience in interpreting MRA and CTA evaluated all MR and CT images for arterial stenosis or other pathology. All images were evaluated without knowledge of further workup.

\section{Measurement of Quality of life}

Health related quality of life was assessed using a self-administrated questionnaire sent to all patients at the time of randomization and 2 weeks, 3 months, and 6 months after the initial imaging test. The questionnaires contained the EuroQol-5D (EQ-5D), the Rating Scale (RS), the generic Medical Outcomes Study 36-Item Short Form Health Survey (SF-36), and the disease specific VascuQol.

The EQ-5D covers five different health dimensions including mobility, selfcare, usual activities, pain and discomfort, and anxiety and depression, which give a total of 243 different health states. Using a published population-based utility function, a single index score was calculated for each patient (18). A value of 0 equals death and a value of 1 equals maximum health.

The RS is a valuative instrument and consists of one question in which the patient was asked to rate his or her current state of health on a scale from 0 to 100 , where 0 represents death and 100 perfect health (19).

The SF-36 is a multi-item scale and covers eight different health dimensions (20). Based on a previous study, we determined that physical functioning, role functioning limitations due to physical problems, bodily pain, and general health were the relevant dimensions to describe the health status of PAD (21). Each dimension is valued on a 100-point scale, in which 0 means death and 100 indicates maximum health.

The VascuQol is a disease specific descriptive quality of life instrument especially for patients with peripheral arterial disease and contains five domains (activity, symptom, pain, emotion, and social functioning) (22). These 5 domains give a total score, which is valued on a 7-point scale, in which 1 means poor quality of life and 7 indicates maximum health.

For each patient we compared the scores of the different quality of life measures at 2 weeks, 3 months, and 6 months follow-up with the baseline score of that particular measure, which resulted in a mean improvement for each quality of life measure. We used standard rules for item recoding, treatment of missing items, and scoring $(18-20,22)$.

\section{Measurement of Costs}

For the cost analysis, we collected information concerning all relevant items of medical care (i.e. diagnostic and therapeutic) used by each patient during the entire trial. The cost of diagnostic imaging included the initial imaging test, all additional vascular imaging, and the associated hospital admissions. The therapeutic cost included costs for percutaneous vascular interventions (i.e. percutaneous angioplasty, stent placement, and thrombolysis), vascular surgery (i.e. aortic 
bifurcation reconstruction, bypass surgery, endarterectomy, and amputation), and associated hospital admissions. Futhermore, we assessed the costs for outpatient visits during 6 months follow-up. All costs were computed from the hospital perspective according to the Dutch guidelines for cost calculations in health care (23).

Diagnostic costs can be divided in directly and non-directly assignable costs.

Directly assignable costs include personnel costs, material costs such as film, and equipment costs. Personnel costs were computed using the measured time spent on a diagnostic imaging test for each involved personnel-category and the mean wage rates from our hospital. Social security of $37 \%$ of the wage was added in accordance with national guidelines. Costs of materials used in diagnostic procedures were based on cost prices and summed. The annuitized costs (24) of the radiological equipment and the annual equipment servicing costs were summed and divided by the proportion of the total available room time $(80 \%$ of a 40 hour work-week) $(23,24)$. Costs were discounted at a rate of $3 \%$ per annum (25).

Non-directly assignable costs include costs of supporting departments, housing costs, and overhead costs. Information on costs of supporting departments was obtained from records of our Financial and Economics Department. The costs for housing were computed for the involved radiological rooms by multiplying the surface space with the housing costs of 204 euros per $\mathrm{m}^{2}$ per year. The overhead costs for MRA, DUS, and CTA were estimated to be $15 \%$ of directly assignable costs (23).

The costs of percutaneous vascular interventions were measured and calculated in a similar fashion. We obtained unit costs of surgery from another study with a comparable study domain and setting to calculate an overall cost per patient per surgical procedure (26). For a limited number of surgical procedures performed in our study, the unit costs were not available from this article and we had to estimate these costs using the published values as starting point (personal communication van Sambeek, MD, PhD, 2002). The number of days of hospital admission and the number of outpatient visits were collected, and the associated costs were calculated using national estimates of hospital admission, intensive care unit admission, and outpatient visits (23). All costs were reported in euros for the year 2002 (the exchange rate was 0.75 euro per US dollar, Dec, 2004).

\section{Measurement of Clinical Utility}

We assessed the therapeutic confidence of vascular radiologists and surgeons during the weekly vascular conference where the findings of the initial imaging test were discussed and each clinician was asked to rate his/her individual confidence in making a well-founded therapeutic choice on a ten-point rating scale. To adjust for variability in using the rating scale we normalized scores from each physician (27).

Furthermore, we measured the recommendations for additional imaging (DUS, digital subtraction angiography (DSA), MRA, or CTA) during the vascular conference. Any additional vascular imaging test performed within 60 days after the initial test was noted. In addition, all additional vascular imaging tests performed during 6 months follow-up were collected. 


\section{Measurement of Functional Patient Outcames}

The brachial, dorsal pedal, and posterior tibial arterial systolic pressure were assessed by a blood pressure cuff and continuous-wave Doppler ultrasound, both before starting and immediately after completion of the treadmill test, to determine resting and post-exercise ankle-brachial indices (ABI). To calculate the $A B I$ the highest ankle pressure was divided by the highest brachial pressure. A treadmill test, based on a standard constant-load protocol, was performed to assess the maximum walking distance (MWD). The patients walked until they had to stop due to leg pain or they reached the time limit. Both $A B I$ and MWD were measured at baseline and after 6 months follow-up.

Furthermore, we assessed the change in clinical status during 6 months follow-up. For this purpose we used the criteria for reporting significant change in clinical status according to Rutherford (28). These criteria are a combination of standard clinical categories with objective ankle-brachial indices.

Improvement in $\mathrm{AB}$ I and change in clinical status during the trial period was assessed for the treated leg only. If both legs or neither leg were treated, we selected the leg with the most severe symptoms at baseline. In case a patient had the same symptoms of both legs at baseline, we selected a leg at random.

\section{Statistical Analyses}

For each moment in time we calculated the response-rate of the quality of life questionnaires. Furthermore, we entered $20 \%$ of both the quality of life data and the data of the case record form twice in the database to calculate the entry error.

The intention of the study was to demonstrate cost-savings for the diagnostic work-up while quality of life and other patient outcomes are not detrimental affected. The sample size calculation was based on quality of life outcomes because we felt it would be essential not to miss a clinically relevant difference in this outcome. With adequate treatment approximately $40-50 \%$ of patients could be expected to have a substantial improvement of their symptoms after 6 months as measured by the physical functioning and pain attributes on the SF-36 (21). A percentage difference of $10 \%-15 \%$ would be considered clinically relevant. A sample size of 500 would be adequate to avoid missing a percentage difference of at least $12.5 \%$ if $45 \%$ of patients improve.

The results were analyzed according to the intention-to (-diagnose-and)-treat principle. For continuous variables, statistical significance of differences between the 3 groups was evaluated using ANOVA. The statistical significance of differences in dichotomous variables between the 3 groups was assessed using the chi-squared test. We determined the statistical significance of differences in improvement in primary and secondary outcomes between the 3 groups with multivariable and logistic regression. Differences in improvement between the 3 groups are presented with adjustment for predictive baseline characteristics, learning curve of physicians, and hospital setting. Based on previous studies (29) and on clinical experience we assumed that severity of disease (critical ischemia vs claudication), renall disease 
(i.e. renal insufficiency and renal transplantation), cerebrovascular disease, cardiac disease, and diabetes mellitus at baseline were potentially predictive for the outcomes. To adjust for the learning curve of the physicians we included the rank order of the initial imaging tests in the regression analysis. We expressed the rank order by ranking the dates when the initial imaging tests were performed. To analyze the improvement in quality of life, $\mathrm{ABI}$, and maximum walking distance during followup we also adjusted for the baseline scores of these outcome measures. A one-way sensitivity analysis was performed for the diagnostic costs by exploring a range of $50 \%$ to $200 \%$ of the investment costs of radiology equipment.

For all outcome measures we used mean imputation for missing values. A Pvalue of 0.01 was considered statistically significant for the quality of life outcomes and the costs because multiple measures were tested within these groups of outcomes. For other tests a significance level of 0.05 was used. Calculations were performed with SPSS 11.0 for Windows (SPSS Inc., Chicago, II).

\section{Results}

Of 984 patients assessed for eligibility, 514 patients were enrolled, and 470 were excluded because they did not fulfill all inclusion criteria $(n=210)$, were not asked to participate $(n=70)$, refused to participate $(n=18)$, needed an acute intervention $(n=141)$, or had contraindications for MRA or CTA $(n=31)$ (Figure 1). Of the 258 patients assigned to MRA 249 actually underwent MRA. Digital subtraction angiography was performed in two patients because of unexpected claustrophobia when they were confronted with the MR scanner and in one patient because an acute intervention was necessary due to progressive disease. One patient underwent CTA because of logistical problems. DUS was performed in one patient because this patient did not fit in the MR scamner and one patient because of claustrophobia. Three patients did not undergo MRA or any other diagnostic or interventional procedure. One patient died prior to the imaging test and two patients decided to delay the diagnostic workup for at least another year and therefore we had no data available for these patients. Of the 177 patients assigned to DUS 173 actually underwent DUS. One patient received MRA due to logistical problems. Three patients did not undergo DUS or any other diagnostic or interventional procedure because two patients died prior to the imaging test and one patient was diagnosed with a lethal disease and withdrew from the study. Of the 79 patients allocated to CTA all underwent CTA. 
Figure 1 Flow diagram

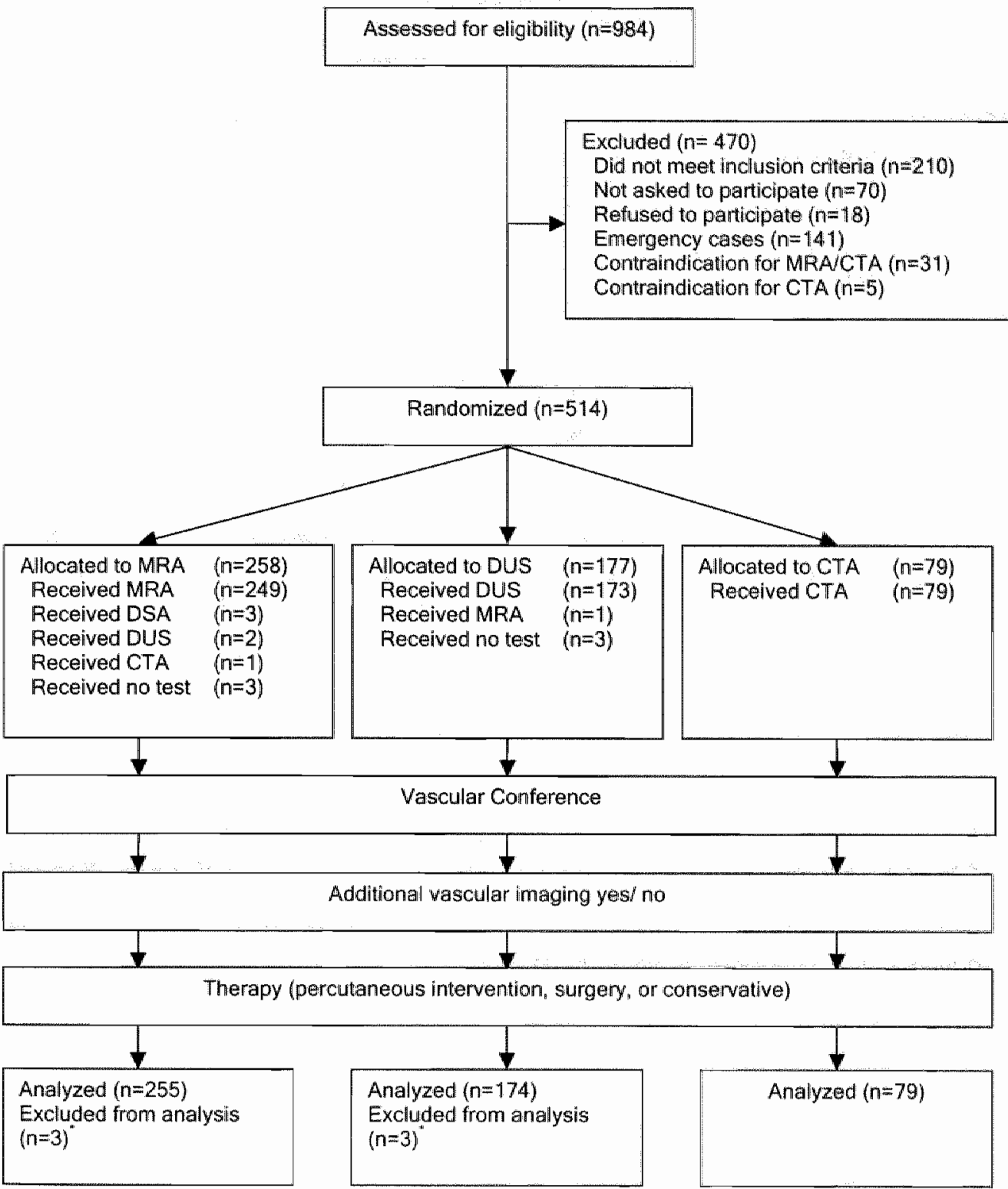

Flow diagram illustrates the reasons for exclusion, random assignment of patients to diagnostic test groups, the diagnostic tests that the patients actually underwent, schematic representation of follow$\mathrm{up}_{\mathrm{a}}$ and actual number of patients included in the analysis. * These patients did not undergo any diagnostic or therapeutic intervention and did not have any data available. 


\begin{tabular}{lllll}
\hline Characteristics & MRA & DUS & CTA & P-value \\
\hline Ade. $y^{*}$ & $64(11)$ & $64(11)$ & $64(12)$ & 1.0 \\
Male sex & $168(66)$ & $122(70)$ & $50(63)$ & 0.5 \\
Diabetes mellitus & $67(26)$ & $30(17)$ & $17(22)$ & 0.09 \\
Hyperlipidemia & $123(51)$ & $77(49)$ & $41(52)$ & 0.9 \\
Simoking & $114(45)$ & $74(43)$ & $31(39)$ & 0.7 \\
Arterial hypertension & $127(50)$ & $79(45)$ & $40(51)$ & 0.6 \\
Cardiac disease & $102(40)$ & $63(36)$ & $20(25)$ & 0.06 \\
Cerebrovascular disease & $41(16)$ & $27(16)$ & $14(18)$ & 0.9 \\
Renal insufficiency & $16(7)$ & $9(5)$ & $1(1)$ & 0.09 \\
Renal transplantation & $9(4)$ & $1(1)$ & $1(1)$ & 0.09 \\
Previous revascularisation & $92(36)$ & $60(35)$ & $30(38)$ & 0.9 \\
Amputation & $10(4)$ & $4(2)$ & $5(6)$ & 0.3 \\
Critical Ischemia & $38(15)$ & $20(12)$ & $14(18)$ & 0.4 \\
ABPI at rest (mean,(SD)) & $0.61(0.20)$ & $0.60(0.20)$ & $0.62(0.17)$ & 0.9 \\
ABPI after exercise & $0.43(0.21)$ & $0.41(0.23)$ & $0.40(0.26)$ & 0.6 \\
Quality of life (mean,(SD)) & & & & \\
EQ-5D & $0.49(0.30)$ & $0.55(0.26)$ & $0.58(0.24)$ & 0.03 \\
Rating scale & $55(21)$ & $60(20)$ & $59(21)$ & 0.06 \\
SF-36 Physical function & $37(22)$ & $40(20)$ & $38(18)$ & 0.4 \\
SF-36Role physical & $34(40)$ & $35(40)$ & $34(40)$ & 1.0 \\
SF-36Badily pain & $44(22)$ & $45(21)$ & $45(21)$ & 0.8 \\
SF-36 General health & $48(21)$ & $53(21)$ & $52(21)$ & 0.02 \\
VascuQal & $3.7(1.2)$ & $3.7(1.1)$ & $3.8(1.2)$ & 0.4 \\
\hline
\end{tabular}

Data are numbers of patients and percentages in parentheses.

${ }^{\dagger}$ Mild renal insuffiency or hemodialysis with permission of the nephrologist to undergo a CTA.

Table 1 shows baseline characteristics of participants according to imaging strategy. The EQ-5D and the domain general health of the SF-36 showed significantly lower values in the MRA group compared to the other 2 groups. The other baseline characteristics were comparable among the 3 groups. We found an error percentage of $0.9 \%$ for double entry of the quality of life data and an error percentage of $0.7 \%$ for double entry of the case record form data.

\section{Quality of Life}

The response rate of the quality of life questionnaires was $99 \%$ at baseline, $93 \%$ at 2 weeks, $89 \%$ at 3 months, and $89 \%$ at 6 months follow-up. The improvement in all quality of life measures from baseline to 2 weeks, 3 months and 6 months follow-up was not statistically significant between the groups (Table 2). However, there was a consistent difference in one direction among all (except one) quality of life measures of slightly more improvement in the CTA group compared to the MRA group. 


\begin{tabular}{|c|c|c|c|c|}
\hline \multirow{2}{*}{\multicolumn{2}{|c|}{ Measure of Quality of Lifie }} & \multicolumn{3}{|c|}{ Adjusted Mean Difference $(95 \% \mathrm{Cl})^{*}$} \\
\hline & & \multirow{2}{*}{$\frac{\text { MRA vs DUS }}{-0.04 \cdot(-0.10 \text { to } 0.01)}$} & \multirow{2}{*}{$\frac{\text { MRA vS CTA }}{-0.02(-0.10 \text { to } 0.06)}$} & \multirow{2}{*}{$\frac{\text { DUS US CTA }}{0.02(-0.07 \text { to } 0.12)}$} \\
\hline EuroQol-5D & 2 weeks & & & \\
\hline & 3 months & $0 .(-0.05$ to 0.06$)$ & $-0.05(-0.13$ to 0.04$)$ & $-0.05(-0.15$ to 0.05$)$ \\
\hline & 6 months & $0 .(-0.06$ to 0.05$)$ & $-0.02(-0.11$ to 0.06$)$ & $-0.02(-0.12$ to 0.08$)$ \\
\hline \multirow[t]{3}{*}{ SF36-Physic funct } & 2 weelks & $-4 \quad(-6$ to 5$)$ & $-4(-8$ to 1$)$ & $1(-5$ to 6$)$ \\
\hline & 3 months & $0 \quad(-5$ to 5$)$ & $-9(-16$ to -2$)$ & $-9(-17$ to 0$)$ \\
\hline & 6 months & $-1 \quad(-6$ to 4$)$ & $-4(-11$ to 4$)$ & $-3(-11$ to 6$)$ \\
\hline \multirow[t]{3}{*}{ SF36-Role physical } & 2 weeks & $-3(-10$ to 3$)$ & $-3(-13$ to 6$)$ & $0(-12$ to 12$)$ \\
\hline & 3 months & $-1 \quad(-9$ to 6$)$ & $-7)(-18$ to 5$)$ & $-5(-19$ to 8$)$ \\
\hline & 6 months & $-6(-13$ to 2$)$ & $-11(-23$ to 0$)$ & $-5(-19$ to 8$)$ \\
\hline \multirow[t]{3}{*}{ SF36-Bodily pain } & 2 weeks & $-3(-6$ to 1$)$ & $-5(-10$ to 1$)$ & $-2(-8$ to 4$)$ \\
\hline & 3 months & $0 \quad(-5$ to 5$)$ & $-6(-13$ to 1$)$ & $-6(-15$ to 3$)$ \\
\hline & 6 months & $-1 \quad(-6$ to 4$)$ & $-3(-11$ to 5$)$ & $-2(-11$ to 8$)$ \\
\hline \multirow[t]{3}{*}{ SF36-General health } & 2 weeks & $-1 \quad(-4$ to 2$)$ & $2(-3$ to 7$)$ & $4(-2$ to 9$)$ \\
\hline & 3 months & $0 \quad(-4$ to 3$)$ & $-4(-9$ to 1$)$ & $-3(-10$ to 3$)$ \\
\hline & 6 months & $-2(-6$ to 2$)$ & $-1(-6$ to 5$)$ & $1(-5$ to 8$)$ \\
\hline \multirow[t]{3}{*}{ Vascuqol } & 2 weeks & $-0.1(-0.3$ to 0.1$)$ & $-0.1(-0.3$ to 0.1$)$ & $0(-0.3$ to 0.2$)$ \\
\hline & 3 months & $0 .(-0.2$ to 0.3$)$ & $-0.2(-0.6$ to 0.2$)$ & $-0.2(-0.7$ to 0.2$)$ \\
\hline & 6 months & $-0.1(-0.3$ to 0.2$)$ & $-0.2(-0.6$ to 0.2$)$ & $-0.1(-0.5$ to 0.4$)$ \\
\hline \multirow[t]{3}{*}{ Rating Scale } & 2 weeks & $2(-1$ to 5$)$ & $-2(-7$ to 3$)$ & $-4(-10$ to 2$)$ \\
\hline & 3 months & o $(-4$ to 4$)$ & $-4(-10$ to 2$)$ & $-4(-11$ to 3$)$ \\
\hline & 6 months & $0 \quad(-4$ to 5$)$ & $-2(-8$ to 5$)$ & $-2(-10$ to 6$)$ \\
\hline
\end{tabular}

For each quality of life measure, the baseline score was subtracted from the score at follow-up to yield the improvement scores. The improvement scores were compared across the groups.

* Adjusted for baseline quality of life scorre, severity of disease, diabetes mellitus, renal disease, cerebrovascular disease, cardiac disease at baseline, hospital, and rank order.

"Negative difference indicates that the MRA group has a smaller improvement of quality of life than the DUS or CTA group and vice versa. ${ }^{*}$ Negative difference indicates that the DUS group has a smaller improvement of quality of life than the CTA group and vice versa. Minimum number of paired observations in MRA group was 244, in DUS group 170, and in CTA group 74,

\section{Costs}

The mean unit cost of the individual imaging tests was 104 euros (SD 38) for all DUS tests, 472 euros (SD 133) for all MRAs, and 163 euros (SD 18) for all CTAs performed during the trial. For the additional vascular imaging tests, the mean unit cost for all diagnostic DSAs (including hospital stay) was 1207 euros (SD 542). The total diagnostic costs per patient were 206 euros higher in the DUS group compared to the CTA group, which was not a statistically significant difference when considering the multiple comparisons that we performed $(P=0.04)$. However, the total diagnostic costs per patient were significantly higher in the MRA group compared to the DUS group (difference $138 ; 95 \% \mathrm{Cl} 31$ to $245 ; P=0.01$ ) and CTA group 
(difference $344 ; 95 \% \mathrm{Cl} 182$ to 506; $P<0.001 ;$ Table 3). This increase in diagnostic costs was not caused by more costs for additional imaging, but was caused by the higher unit costs of the initial imaging test in the MRA group (Table 3). With one-way sensitivity analysis only the difference in total diagnostic costs between the MRA and DUS group was sensitive to variation of the investment costs of radiological equipment with a range of $50 \%$ to $200 \%$ (Table 4). If the investment costs of MR equipment were $50 \%$ than the baseline estimate, the difference in total diagnostic costs between the MRA and DUS group was not statistically significant anymore, but the difference between the MRA and CTA group was still significant. Note that the difference in diagnostic costs between the CTA and DUS group also changed with varying the investment costs of MR equipment. This is explained by the additional MRA exams in the DUS group. The costs for percutaneous interventions and surgical procedures were not statistically significant between the groups when considering the multiple comparisons that we performed (Table 3). The costs for outpatient visits were comparable between the groups (Table 3 ). The total costs including diagnostic, therapeutic, and outpatient visit costs were significantly lower in the CTA group compared to the MRA group $(P=0.001)$ and compared to the DUS group $(P=0.01)$. The total costs were comparable between the MRA and DUS group ( $P=0.6$; Table 3$)$.

\section{Therapeutic Confidence and Additional Imaging}

The mean therapeutic confidence was 8.1 (SD 1.4) for MRA, 8.0 (SD 1.1) for CTA, and 7.5 (SD 1.7) for DUS. The therapeutic confidence was significantly higher for MRA compared to DUS (difference $0.8 ; 95 \% \mathrm{Cl} 0.5$ to $1.1 ; P<0.001$ ) and for CTA compared to DUS (difference $1.0 ; 95 \% \mathrm{Cl} 0.4$ to $1.5 ; P<0.001$ ).

Within 60 days after the initial imaging test on average more additional vascular imaging tests per patient were performed in the DUS group compared to the MRA group ( $23 \%$ versus $8 \% ;, 0<0.001)$ and compared to the CTA group ( $23 \%$ versus $6 \% ; P=0.01)$. During the total follow-up of 6 months this difference was $19 \%$ more imaging tests per patient for DUS compared to MRA $(P=0.001)$ and $22 \%$ for DUS compared to CTA $(P=0.03)$. There were no significant differences in the confidence or the number of additional vascular imaging tests between the MRA group and the CTA group. 


\begin{tabular}{|c|c|c|c|}
\hline \multirow[t]{2}{*}{ Cost component } & \multicolumn{3}{|c|}{ Adjusted Mean Difference $(95 \% \mathrm{Cl})^{*}$} \\
\hline & MRA vs DUS & MRA ws CTA' & DUS YS. CTA* \\
\hline $\begin{array}{l}\text { Additional Imaging within } 60 \text { days } \\
\text { (cost initial test not included) }\end{array}$ & $-158(-241$ to -75$)$ & $-39(-1641086)$ & $119(-31$ to 269$)$ \\
\hline $\begin{array}{l}\text { Additional Imaging within } 60 \text { days } \\
\text { (cost initial test included) }\end{array}$ & $188(103$ to 273$)$ & $308(180$ to 37$)$ & $120(-34$ to 273$)$ \\
\hline Additional Imaging during follow-up & $-50(-111$ to 10$)$ & $36 \quad(-56$ to 127$)$ & $86(-23$ to 196$)$ \\
\hline Total Diagnostic costs $\$$ & $138(31$ to 245$)$ & $344(182$ to 506$)$ & $206(13$ to 399$)$ \\
\hline Percutaneous interventions " & $120(-209$ to 450$)$ & $337(-160$ to 835$)$ & $217(-377$ to 811$)$ \\
\hline Surgical procedures & $18(-909$ to 945$)$ & 1853 (453 to 3252$)$ & 1834 (162 to 3506$)$ \\
\hline Outpatient visits & $-9 \quad(-24$ to 5$)$ & $(-20$ to 23$)$ & $11(-15$ to 36$)$ \\
\hline Total costs & $267(-693$ to 1228$)$ & $2535(1085$ to 3985$)$ & 2268 (535 to 4000$)$ \\
\hline
\end{tabular}

Only diagnostic and therapeutic costs of PAD during: 6 months follow-up were included.

Purchasing power in euros for the year 2002. Exchange rate was 0.90 euro per US dollar, Jan, 2002 .

* Adjusted for severity of disease (critical ischemia vs claudication), nenal disease, cerebrovasculan" disease, cardiac disease, diabetes mellitus at baseline, hospital and rank order.

* Positive cost difference indicates that MRA is more costly than DUS or CTA and vice veirsa.

* Positive cost difference indicates that DUS is more costly than CTA and vice versa.

Diagnostic cost include hospital stay, if necessary, for pre-procedural work-up and post-procedural observation. "Including hospital stay.

Table 4 One-way Sensitivity Analysis of the Difference in Total Diagnostic Costs Between Groups

\begin{tabular}{|c|c|c|c|}
\hline \multirow[t]{2}{*}{ Total Diagnostic costs } & \multicolumn{3}{|c|}{ Adjusted Mean Difference $(95 \% \mathrm{Cl})^{*}$} \\
\hline & MRA vs. DUS ${ }^{\dagger}$ & MRA ws. CTA ${ }^{\dagger}$ & DUS vs. GTA \\
\hline Baseline & $138 \quad$ (31 to 245$)$ & $344(182$ to 506$)$ & $206(13$ to 399$)$ \\
\hline Investment cost MRA $50 \%$ & $69(-3710175)$ & 259 (99 to 419$)$ & 190 ( 1 to 381$)$ \\
\hline Investment cost MRA $200 \%$ & $279(169$ 10 390) & $516(349$ to 683$)$ & $237(37$ to 436$)$ \\
\hline Investment cost DUS $50 \%$ & $152 \quad(45$ to 259$)$ & $344(182$ to 506$)$ & $192(1$ to 386$)$ \\
\hline Investment cost DUS $200 \%$ & $111 \quad(4$ to 218$)$ & $34.4(182$ to 506$)$ & $233(40$ to 426$)$ \\
\hline tnvestment cost CTA $50 \%$ & $138(31$ to 245$)$ & $353(191$ to 515$)$ & $214(20$ to 407$)$ \\
\hline Inwestment cost CTA $200 \%$ & $138(31$ to 245$)$ & $328(166$ to 490$)$ & $189(5$ to 382$)$ \\
\hline
\end{tabular}

Exploring range of $50 \%$ to $200 \%$ of the investment costs of radiological equipment.

Baseline is all investment costs of radiological equipment fixed at $100 \%$.

Purchasing power in euros for the year 2002.

* Adjusted for severity of disease (critical ischemia vs. claudication), renal disease, cerebrovascular disease, cardiac disease, diabetes mellitus at baseline, hospital, and rank order.

$\dagger$ Positive cost difference indicates that MRA is more costly than DUS or CTA and vice versa.

* Positive cost difference indicates that DUS is more costly than CTA and vice versa. 


\section{Ankle-Brachial index, Maximum Walking Distance, and Clinical Status}

The difference in improvement in $A B I, M W D$, and clinical status from baseline to 6 months follow-up was not statistically significant between the groups (Table 5). For $\mathrm{ABI}$. MWD, and clinical status there was a consistent difference in one direction of slightly more improvement in the CTA group compared to the MRA group.

Table 5 Differences in Improventent of Patient Outcomes between the groups

\begin{tabular}{lcccc}
\hline & \multicolumn{4}{c}{ Adjusted Mean Difference $\left(95 \% \mathrm{Cl}^{*}\right.$} \\
Patient Outcome & MRA vs DUS $^{*}$ & MRA vs. CTA & DUS vs. CTA \\
\hline ABI at rest & $0.01(-0.04$ to 0.05$)$ & $-0.04(-0.11$ to 0.02$)$ & -0.05 & $(-0.13$ to 0.03$)$ \\
ABI after exercise & $0.01(-0.04$ to 0.07$)$ & $0(-0.08$ to 0.08$)$ & $-0.01 \quad(-0.11$ to 0.08$)$ \\
Maximum Walking Distance & $-4(-19$ to 10$)$ & $-14(-36$ to 8$)$ & $-10 \quad(-36$ to 17$)$ \\
Change in Clinical Status* & $7(-8$ to 22$)$ & $-7(-17$ to 4$)$ & $14(-5$ to 32$)$ \\
\hline
\end{tabular}

Paired observations in MRA group were 244, in DUS group 170, and in CTA group 74 .

In MRA group $46 \%$ completed the maximum walking time (mean 273 meter), in Duplex group $57 \%$, and in CTA group $53 \%(p=0.1)$. Data are means. "Adjusted for baseline scores of $A B I$ or maximum walking distance, severity of disease (critical ischemia vs. claudication), renall disease, cerebrovascular disease, cardiac disease, diabetes mellitus at baseline, hospital, and rank order.

Only measured for the treated leg during 6 months follow-up.

"Negative difference indicates that the MRA group has less improvement than the DUS or

CTA group and vice versa. $\S$ Negative difference indicates that the DUS group has less improvement than the CTA group and vice versa. "Data are percentages.

\section{Discussion}

We performed a multicenter randomized controlled trial to evaluate the costs and effects of non-invasive imaging strategies. Both MRA and CTA provided similar improvement in quality of life and functional patient outcomes as DUS, but provided higher confidence and less additional vascular imaging tests compared to DUS. Furthermore, the total costs were similar for MRA and DUS, but CTA incurred lower total costs during 6 months follow-up compared to MRA and DUS. The results suggest that both MRA and CTA are clinically more useful than DUS and that CTA leads to cost-savings compared to both MRA and DUS in the initial imaging evaluation of patients with peripheral arterial disease. The final decision which test should be implemented in routine clinical practise in a particular setting also depends on local expertise, availability of equipment, and considerations concerning ionizing radiation and renal insufficiency.

The mean diagnostic costs were significantly higher in the MRA group compared to the DUS and CTA group which is explained by the higher costs of the initial imaging test. MRA is more expensive than DUS and CTA due to higher investment costs, construction costs, costs for the contrast agent, and personnel costs. A reduction in the investment costs of MR equipment would make the difference in total diagnostic costs of MRA compared to the DUS strategy 
insignificant, but the CTA strategy would still be cost-saving compared to MRA. Furthermore, the total costs in the CTA group were significantly lower than in the MRA and DUS group.

We found that the therapeutic confidence for MRA and CTA was higher than for DUS. A probable explanation is that both MRA and CTA provide a precise roadmap for planning treatment whereas DUS provides interpreted data on a schematic drawing. Probably due to the lower confidence in DUS, the physicians requested additional vascular imaging tests more frequently in the DUS group than in the MRA and CTA group.

A cohort study has traditionally been used for the evaluation of new diagnestic imaging tests by performing both the new test and the reference test in all patients to determine the sensitivity and specificity. For DUS a sensitivity of $88 \%$ and a specificity of $95 \%$ have been reported (3). For both MRA and CTA a sensitivity between $91-98 \%$ and a specificity between $92-99 \%$ have been reported (4-11). These results are, however difficult to translate into a meaningful clinical decision with respect to which diagnostic strategy should actually be implemented. A decision about the usefulness of a diagnostic strategy requires either a decision analysis or a randomized controlled trial (12). Although randomized controlled trials are not frequently used to evaluate diagnostic tests, we found our pragmatic randomized trial to be both feasible and inexpensive $(13,30-33)$.

We acknowledge several limitations of our study. Although eligible patients were randomized between MRA and DUS in three hospitals and between MRA and CTA in one hospital we also compared the DUS group with the CTA group. CTA is a relatively new test and was only performed in one hospital. We feel that the comparison between DUS and CTA is valid because both groups were randomized in one study with the same inclusion and exclusion criteria. Furthermore, the baseline characteristics were comparable between these two groups and we adjusted for predictive variables.

Another limitation of the study was that although patients were randomized, there were differences between the MRA group and the other two groups at baseline in EQ-5D and the dimension general health of SF-36. To calculate the difference in improvement of quality of life we adjusted for the baseline quality of life scores. There were minor differences between the groups at baseline in diabetes mellitus, cardiac disease, renal disease, and critical ischemia. These baseline differences were not statistically significant, but we felt it would be prudent to adjust for predictive baseline variables that may lead to differences in outcomes $(34,35)$. Therefore, adjustment for potentially predictive variables in a multivariable or logistic regression was used to correct the estimates of the outcomes for any imbalance that by chance may have occurred between the randomized groups. Furthermore, imaging techniques were different between the hospitals. For this reason we adjusted for hospital setting in the regression analysis.

A possible limitation was that patients and physicians were not blinded for group allocation. At the same time, the goal of our study was to evaluate the outcomes of the diagnostic tests as they are used in routine clinical practice. Patients could not be blinded and blinding of the treating physicians by for example, transferring the diagnostic information to a schematic drawing would have introduced 
an artificial step that could have affected diagnostic interpretation and therapeutic planning: Furthermore, although schematic drawings are used in routine clinical practice as adjunct, they are not used solely when the imaging test provides a good roadmap.

Finally, the costs were calculated from a hospital perspective instead of a societal perspective. There is international consensus that an economic evaluation should be performed from the societal perspective(36). A societal perspective implies that not only the costs within the healthcare sector, but also the direct (i.e. patient costs) and indirect costs (i.e. costs of production losses) outside the healthcare sector have to be included in the cost analysis (25). in our study we chose for the hospital perspective because other studies which assessed the costs related to the management of PAD showed that patient costs were low in both the Dutch and US settings $(37,38)$. Furthermore, in the setting of PAD the costs of production losses are negligible since most patients are retired (39).

In conclusion, the results suggest that both MRA and CTA are clinically more useful than DUS and that CTA leads to cost-savings compared to both MRA and DUS in the initial imaging evaluation of patients with peripheral arterial disease. 


\section{References}

1. Meijer WT, Hoes AW, Rutgers D, Bots ML, Hofman A, Grobbee DE. Penipheral arterial disease in the elderly: The Rotterdam Study. Arterioscler Thromb Vasc Biol 1998:18(2):185-192.

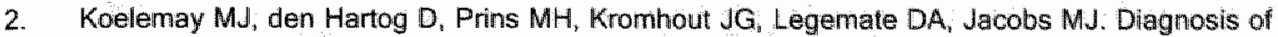
arterial disease of the lower extremities with duplex ultrasonography. $\mathrm{Br} J$ Surg 1996;83(3):404409.

3. Visser $\mathrm{K}$, Hunink MG. Peripheral arterial disease: gadolinium-enhanced MR angiography versus color-guided duplex US--a meta-analysis. Radiology 2000;216(1):67-77.

4. Ho KY, Leiner $T$, de Haan MW, Kessels AG, Kitslaar PJ, van Engelshoven JM. Peripherall vascular tree stenoses: evaluation with moving-bed infusion-tracking MR angiography. Radiology 1998;206(3):683-692.

5. Martin ML, Tay KH, Flak $B_{\text {, }}$ et al. Multidetector CT Angiography of the Aortolliac System and Lower Extremities: A Prospective Comparison with Digital Subtraction Angiography. AJIR Am J Roentgenol 2003;180(4):1085-1091.

6. Meaney JF, Ridgway JP, Chakraverty $S$, et al. Stepping-table gadolinium-enhanced digital subtraction MR angiography of the aorta and lower extremity arteries: preliminary experience. Radiology 1999;211(1):59-67.

7. Ofer A, Nitecki SS, Linn S, et al. Multidetector CT angiography of peripheral vascular disease: a prospective comparison with intraarterial digital subtraction angiography. AuR Am J Roentgenol 2003:180(3):719-724.

8. Swan JS, Carroll TJ, Kennell TW, et al. Time-resolved three-dimensional contrast-enhanced MR angiography of the peripheral vessels. Radiology 2002;225(1):43-52.

9. Tins $B$, Oxtoby $J$, Patel $S$. Comparison of $C T$ angiography with conventional arteriall angiography in aortoiliac occlusive disease. $\mathrm{Br} J$ Radiol 2001;74(879):219-225.

10. Willmann $J K$, Wildermuth $S$, Pfammatter $T$, et al. Aortoiliac and renal arterles: prospective intraindividual comparison of contrast-enhanced three-dimensional MR angiography and multidetector row CT angiography. Radiology 2003;226(3):798-811.

11. Catalano C FF, Laghi A, Napoli A, Bezzi M, Pediconi F, Danti M, Nofroni I, Passariello R. Infrarenal Aortic and Lower-Extremity Arterial Disease: Diagnostic Performance of MultiDetector Row CT Angiography. Radiology 2004;231(2):555-563.

12. Bossuyt PM, Lijmer JG, Mol BW. Randomised comparisons of medical tests: sometimes invalid, not always efficient. Lancet 2000;356(9244):1844-1847.

13. Hunink MG, Krestin GP. Study design for concurrent development, assessment, and implementation of new diagnostic imaging technology. Radiology 2002:222(3):604-614.

14. McDermott MM, Greenland $P_{1}$ Lilu $K_{\text {, }}$ et al. The ankle brachial index is associated with leg function and physical activity: the Walking and Leg Circulation Study. Ann Intern Med 2002; 136(12):873-883.

15. McDermott $M M$, Greenland $P_{1}$ Litu $K_{\text {r }}$ et al. Leg symptoms in peripheral arterial disease: associated clinical characteristics and functional impairment. Jama 2001;286(13):1599-1606.

16. Guidelines for good clinical practice (GCP) for trials on pharmaceutical products. World Health Organisation 1995:WHO technical report series, no.850, annex 853.

17. Moher D, Schuiz KF, Altman DG. The CONSORT statement: revised recommendations for improving the quality of reports of parallel-group randomized trials. I Am Podiatr Med Assoc $2001 ; 91(8): 437-442$.

18. Dolan P. Modeling valuations for EuroQol health states. Med Care 1997;35(11):1095-1108.

19. Froberg DG, Kane RL. Methodology for measuring health-state preferences-4l: Scaling methods. J Clin Epidemiol 1989;42(5):459-471. 
20. Ware JE; Jr., Sherboume CD. The MOS 36-item short-form health survey (SF-36): I. Conceptual framework and item selection. Med Care 1992;30(6):473-483.

21. Bosch JL, van der Graaf $Y$, Hunink MG. Heatth-related quality of life after angioplasty and stent placement in patients with iliac artery ocdusive disease: results of a randomized controlled elinical trial: The Dutch lliac Stent Trial Study Group Circulation 1999:99(24):3155-3160.

22. Morgan $M B$, Crayford $T$, Murrin $B$, Fraser $S C$, Developing the Vascular Quality of Life Questionnaire: a new disease-specific quality of life measure for use in lower limb ischemia. $U$ Vasc Surg $2001 ; 33(4) 679687$

23. Oostenbrink JB, Koopmanschap MA, Rutten FF. Standardisation of costs: the Dutch Manual for Costing in economic evaluations. Phamacoeconomics 2002;20(7):443-454.

24. Hunink MG, Glasziou PP. Decion making in health and medicine-integrating evidence and values. Cambridge: Cambridge University Press; 2001.

25. Gold MR SJ, Rusell LB, Weinstein MC. Cost-effectiveness in health and medicine. Oxford: Oxford University Press; 1996.

26. Visser $K$, de Vries SO, Kitslaar PJ, van Engelshoven JM, Hunink MG. Cost-effectiveness of diagnostic imaging work-up and treatment for patients with intermittent claudication in The Netherlands. Eur d Vasc Endovasc Surg 2003;25(3):213-223.

27. Adriaensen ME ea. Therapeutic Confidence of Multi-Detector CT Angiography compared with Digital Subtraction Anglography for Peripheral Arterial Disease and its Impact on Additional Imaging Recommendations. In press Radiology.

28. Rutherford RB, Baker JD, Ernst $C$, et al. Recommended standards for reports dealing with lower extremity ischemia: revised version. J Vasc Surg 1997;26(3):517-538.

29. Dormandy لA, Rutherford RB. Management of peripheral arterial disease (PAD). TASC Working Group. TransAtlantic Inter-Society Concensus (TASC). J Vasc Surg 2000;31(1 P4 2):S1-S296.

30. Musset $D$, Parent $F$, Meyer $G$, et al. Diagnostic strategy for patients with suspected pulmonary embolism: a prospective multicentre outcome study. Lancet 2002;360(9349):1914-1920.

31. $\mathrm{Ng} \mathrm{CS}$, Watson CJ, Palmer CR, et al. Evaluation of early abdominopelvic computed tomography in patients with acute abdominal pain of unknown cause: prospective randomised study. Bmj 2002;325(7377):1387.

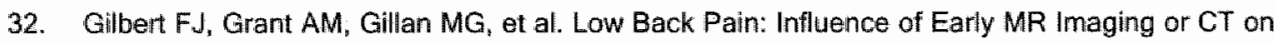
Treatment and Outcome-Multicenter Randomized Trial. Radiology 2004.

33. Jarvik JG, Hollingworth $W$, Martin $B_{n}$ et al. Rapid magnetic resonance imaging vs radiographs for patients with low back pain: a randomized controlled trial. JAMA 2003:289(21):2810-2818.

34. Steyerberg $E W$, Bossuyt PM, Lee KL. Clinical trials in acute myocardial infarction: should we adjust for baseline characteristics? Am Heart ل 2000;139(5):745-751.

35. Pocock SJ, Assmann SE, Enos LE, Kasten LE. Subgroup analysis, covariate adjustment and baseline comparisons in clinical trial reporting: current practice and problems. Stat Med 2002;21(19):2917-2930.

36. Gold MR, Joanna E. Siegel, Louise B. Russell, and Milton C. Weinstein. Cost-effectiveness in Heralth and Medicine. New York: Oxford University Press; 1996.

37. Bosch JL, Tetteroo E. Mali WP, Hunink MG. lliac arterial occlusive disease: cost-effectiveness analysis of stent placement versus percutaneous transluminal angioplasty. Dutch lliac Stent Trial Study Group. Radiology 1998;208(3):641-648.

38. Bosch JL. Haaring C, Meyerovitz MF, Cullen KA, Hunink MG. Cost-effectiveness of percutaneous treatment of iliac artery occlusive disease in the United States. AJR Am J Roentgenol 2000;175(2):517-521.

39. Koopmanschap MA, Rutten FF, van Ineveld $B M$, van Roijen $L$. The friction cost method for measuring indirect costs of disease. J Health Econ 1995;14(2):171-189. 


\section{CHAPTER 6}

Contrast-Enhanced Peripheral MR Angiography at 3.0

Tesla: Initial Experience With a Whole-Body Scanner in Healthy Volunteers

J Magn Reson Imaging (JMRI) 2003; 17: 609-614

Tim Leiner, Marianne de Vries, Romhild Hoogeveen, G. Boudewijn C. Vasbinder, Etienne Lemaire, and Jos M.A. van Engelshoven. 


\section{ABSTRACT}

\section{Purpose}

To report preliminary experience with contrast-enhanced magnetic resonance angiography (CEMRA) of the peripheral arteries on a $3.0 \mathrm{~T}$ whole-body scanner equipped with a prototype body coil.

\section{Materials and Methods}

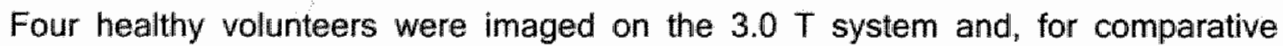
purposes, two of the subjects were also imaged on a commercially availlable $1.5 \mathrm{~T}$ whole-body system. To investigate field strength influence on objective image quallity, signal-to-noise (SN) and contrast-to-noise (CN) ratios were calculated for named vessels from the infrarenal aorta to the ankles at both field strengths. Comparable imaging protocols were used at both field strengths. In addition, two reviewers, blinded for field strength, gave subjective image quality scores (threepoint scale).

\section{Results}

SN and CN ratios were approximately equal on both systems (variation _ $9 \%$ ) for the iliac and proximal upper leg stations. For the popliteal and lower leg stations SN ratios were $36 \%$ and $97 \%$ higher, and $C N$ ratios were $44 \%$ and $127 \%$ higher, at 3.0 $\mathrm{T}$. Subjective image quality at $3.0 \mathrm{~T}$ was substantially better for the distal upper and lower legs.

\section{Conclusion}

Contrast-enhanced peripheral MRA is possible at $3.0 \mathrm{~T}$ when an imaging protocol similar to a current state-of-the-art 1.5 $\mathrm{T}$ protocol is used. Objective and subjective image quality at 3.0 T is comparable for the illac and upper legs but better for the popliteal and lower leg arteries. 


\section{Introduction}

Contrast-enhanced magnetic resonance angiography (CE-MRA) of peripheral arteries using 1.0 and $1.5 \mathrm{~T}$ MR scanners has become a routinely used imaging modality in recent years $(1,2)$. When multiple fields-of-view (FOV) are acquired during first arterial bolus passage of intravenously injected paramagnetic contrast medium, the entire peripheral arterial tree from aorta down to the ankles can be imaged with high accuracy $(3,4)$. Recently, the first $3.0 \mathrm{~T}$ MR systems with wholebody coils have become available for clinical use $(5,6)$. The higher signal-to-noise (SN) ratio at 3.0 T is of potential benefit to peripheral CE-MRA because it offers the possibility to use higher resolution scanning protocols which might enable more reliable detection and grading of peripheral arterial occlusive disease (PAOD). Using a 1.5 T MR scanner, obtaining sufficiently high levels of $\mathrm{SN}$ can be problematic, mainly in the lower legs (7). It has already been shown by Campeau et al (8) that non-enhanced MR angiography has higher SN and better image quality for the depiction of head and neck vasculature at $3.0 \mathrm{~T}$. However, the benefits of a $3.0 \mathrm{~T}$ body coil over a $1.5 \mathrm{~T}$ coil are still unclear for peripheral CE-MRA because a comparative study has not yet been published.

The purpose of this work was to assess the feasibility of CE-MRA of the peripheral arteries at 3.0 T and to compare objective and subjective image quality with that obtained using a similar imaging protocol used at $1.5 \mathrm{~T}$.

\section{Material and Methods}

\section{Subjects}

Four healthy volunteers (three males and one female, 26-33 years old) with no history of symptoms of peripheral arterial disease were examined at $3.0 \mathrm{~T}$. Two of the male volunteers also underwent comparative examinations on a $1.5 \mathrm{~T}$ MR imaging system. Approval of the institutional review board and informed consent of all participants were obtained prior to commencement of the study.

\section{MR Imaging Protocols}

Prior to imaging, a 20 gauge intravenous line was established in an antecubital vein of the right arm to inject contrast medium. For all contrast-enhanced studies the volunteers were injected with $35 \mathrm{~mL}$ gadolinium-DTPA (Magnevist, Schering, Berlin, Germany) administered at a rate of $1.2 \mathrm{~mL} /$ second for the first $15 \mathrm{~mL}$ and $0.8 \mathrm{~mL} /$ second for the remaining $20 \mathrm{~mL}$. After injection of contrast, material tubing and veins were flushed with $25 \mathrm{~mL}$ saline administered at $0.8 \mathrm{~mL} /$ second.

The experiments at 3.0 $\mathrm{T}$ were performed using a commercially available wholebody MR scanner (Intera [software release 8.1] and peripheral MRA software, MobiTrak; Philips Medical Systems, Best, The Netherlands). For signal transmission and reception, a prototype built-in quadrature-body coil was used. The scanning parameters that were used are listed in Table 1. As the Ernst angle decreases due to 
the longer $T 1$ values of gadolinium at higher field strengths, the flip angle was decreased slightly at $3.0 \mathrm{~T}$ compared with $1.5 \mathrm{~T}$. To prescribe the contrast-enhanced scans time-of-flight (TOF), localizer scans were obtained. We used four overlapping FOV of $300 \mathrm{~mm}$. Overlap between adjacent FOVs was set to $30 \mathrm{~mm}$ so that the total coverage in the feethead direction was $1110 \mathrm{~mm}$. Imaging parameters were identical for all FOV. Correct synchronization between injection of contrast medium and start of acquisition was ensured using the real time bolus fluoroscopy capability of the MR scanner (BolusTrak, Philips Medical Systems, Best; The Netherlands). For the first (iliac) station, patients were asked to hold their breath. To increase the vessel to background contrast, nonenhanced scans of all stations were obtained prior to the injection of contrast medium that were subtracted from the enhanced scans after the acquisitions had ended.

To ensure that datasets were as similar as possible, imaging experiments at 1.5 $\mathrm{T}$ were done with the same imaging parameters whenever possible. All experiments were done using a commercially available whole-body MR scanner (Intera [software release 8.1]; Philips Medical Systems, Best, The Netherlands). Although dedicated peripheral coils are routinely used at $1.5 \mathrm{~T}$, these coils were not yet available for $3 T$ at the time of this study. Consequently, for a fair comparison, the system built-in quadrature-body coil was used for signall transmission and reception. The scanning parameters that were used are also listed in Table 1.

Table 1 Imaging Parameters Used at 1.5 and $3.0 \mathrm{~T}$

\begin{tabular}{lll}
\hline & \multicolumn{2}{c}{ Field strength } \\
Parameter & $3.0 \mathrm{~T}$ & $1.5 \mathrm{~T}$ \\
\hline Pulse sequence & Spoiled 3D-GRE & Spoiled 3D-GRE \\
TR (msec) & 3.2 & 3.3 \\
TE (msec) & 1.3 & 1.3 \\
Flip angle (degrees) & 25 & 30 \\
Field of view (mm) & 300 & 300 \\
Matrix & $224 \times 224^{\text {a }}$ & $224 \times 224^{\text {a }}$ \\
Silice thickness (mm) & $3.0^{\mathrm{b}}$ & $3.0^{\mathrm{b}}$ \\
Number of slices & 25 & 25 \\
Voxel size (mm3) & $5.4^{\mathrm{c}}$ & $5.4^{\mathrm{c}}$ \\
Duration (seconds) & 18 & 19 \\
Reception coill used & $\mathrm{Q}-$ body & Q-body \\
\hline
\end{tabular}

1a: Zero-interpolated after acquisition to 512 matrix.

Ib: Zero-interpolated after acquisition to $1.5 \mathrm{~mm}$.

1c: Acquisition duration is per FOV, actual acquisition time is the time needed to image 4 FOV and three times 4 seconds for table movement, 3D-GRE-three-dimensional gradient recalled echo, $Q$ body system builtain quadrature-body coil. 


\section{Image Evaluation}

For image evaluation purposes, the peripheral vascular tree was divided into the following segments infrarenal aorta; left and right common iliac; external iliac; common femoral, superficial femoral (proximal, middle, and distal); popliteal (above and below tibial plateau); anterior tibial (horizontal, proximal, and distal); posterior tibial (proximal and distal); peroneal artery (proximal and distal); and the tibioperoneal trunc. This totaled 35 vascular segments in each volunteer:

Image quality was analyzed in the two subjects for whom comparisons existed between $1.5 \mathrm{~T}$ and $3.0 \mathrm{~T}$ and was quantified objectively by calculating $\mathrm{SN}$ and $\mathrm{CN}$ ratios in all arterial segments. Signal was measured in an intravascular useridefined region-of-interest (ROI) and care was taken to ensure that ROls were placed in the exact same lacation for $1.5 \mathrm{~T}$ and $3.0 \mathrm{~T}$ images. Noise was measured by taking the standard deviation (SD) of air outside the patient, or, if not available, in a region of homogeneous tissue inside the patient corrected for magnitude effects by the Rayleigh correction (9). Image contrast was calculated as the difference between the signal within the artery and that measured in a ROI of the same size in a region immediately adjacent to the vessel. In all MR angiograms signal, contrast, and noise were measured in original partitions. In addition to measuring $S N$ and $C N$, subjective image quality was assessed on a three-point scale. Image quality was scored as 2 when the vessel segment was well interpretable, 1 when the observer thought it was suboptimally interpretable, and 0 when there was poor interpretability (considered to be nondiagnostic). Image quality was assessed by two radiologists experienced in reading MR anglograms and blinded for the field strength at which the experiments were carried out and for each other's results. Results are expressed as the average of all arterial segments analyzed in a perticular FOV as well as corresponding SDs. Subjective interpretability scores (S\|S) are given as the mean of the two observers. 


\section{Results}

All experiments were performed without any adverse events. After injection of contrast material, arteries enhanced intensely at both $1.5 \mathrm{~T}$ and $3.0 \mathrm{~T}$ field strengths. An example of the obtainable image quality is shown in Figure 1.

The results of the $S N, C N$, and image quality measurements are listed in Table 2. For the proximal two stations (aortoiliac and proximal upper leg), SN and CN were unchanged or slightly lower. However, at 3.0 T, SN and CN were substantially higher compared with $1.5 \mathrm{~T}$ for the distal two stations. The differences in SN and CN in the two distal stations were not just quantitative, but also clearly evident in image quality (Fig. 2). Subjective image quality reflected SN and $\mathrm{CN}$ findings and was considered to be nearly equal for the proximal two stations but higher for the distal two stations at $3.0 \mathrm{~T}$ compared with $1.5 \mathrm{~T}$ (Table 2).

Table 2 Signal-to-Noise, Contrast-tom Noise and Subjective Image Quality at 1.5 and $3.0 \mathrm{~T}$

\begin{tabular}{|c|c|c|c|c|}
\hline $\begin{array}{l}\text { Endpoint and } \\
\text { field strength }\end{array}$ & Aortoiliac & $\begin{array}{c}\text { FOV } \\
\text { Proximal upper leg }\end{array}$ & Distal upper leg & Lower leg \\
\hline$S / N 1.5 T$ & $21.9( \pm 2.2)$ & $54.8 \quad( \pm 5.8)$ & $34.7 \quad( \pm 7.4)$ & $22.2( \pm 4.3)$ \\
\hline$S / N 3.0 \mathrm{~T}$ & $21.9( \pm 4.0)$ & $53.3( \pm 11.1)$ & $47.3( \pm 12.4)$ & $43.7( \pm 5.9)$ \\
\hline$\%$ change ${ }^{a}$ & 0 & $-3 \%$ & $+36 \%$ & $+97 \%$ \\
\hline $\mathrm{C} / \mathrm{N} 1.5 \mathrm{~T}$ & $19.0( \pm 1.9)$ & $48.6 \quad( \pm 5.2)$ & $29.4 \quad( \pm 6.8)$ & $16.9( \pm 4.2)$ \\
\hline $\mathrm{C} / \mathrm{N} 3.0 \mathrm{~T}$ & $17.3( \pm 2.2)$ & $46.1( \pm 10.2)$ & $4.2 .2( \pm 12.1)$ & $38.3( \pm 5.4)$ \\
\hline$\%$ change ${ }^{\circ}$ & $-9 \%$ & $-5 \%$ & $+44 \%$ & $+127 \%$ \\
\hline SIS $1.5 \mathrm{~T}$ & $1.6( \pm 0.3)$ & $1.7( \pm 0.2)$ & $1.4( \pm 0.2)$ & $0.6( \pm 0.4)$ \\
\hline SIS $3.0 \mathrm{~T}$ & $1.5( \pm 0.3)$ & $1.8 \quad \pm 0.2)$ & $2.0 \quad( \pm 0.0)$ & $1.8( \pm 0.2)$ \\
\hline
\end{tabular}

FOV = field of view, $S / N=$ signal-to-noise ratio,$C / N=$ contrast-to-noise ratio, SIS = subjective interpretability score (see text for explanation of scale).

a: \% change is expressed as [(value at $3.0 \mathrm{~T}$ - value at $1.5 \mathrm{~T}) /($ value at $1.5 \mathrm{~T})] * 100$. 

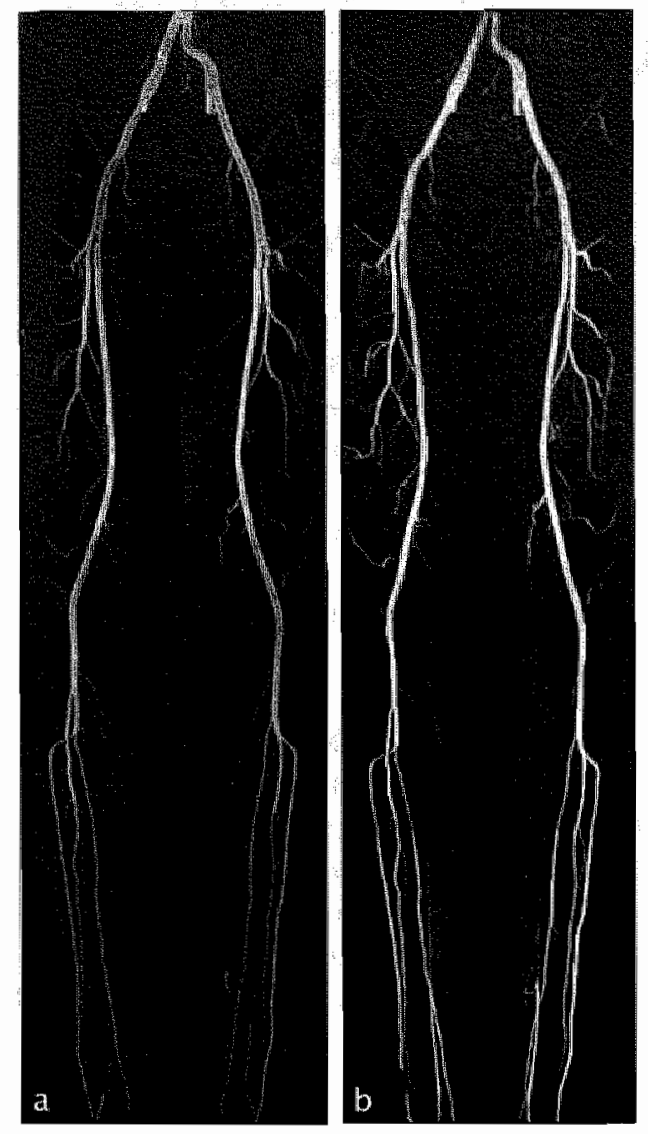

\section{Figure 1}

Peripheral MR angiograms in a 31 -year-old healthy volunteer at $1.5 \mathrm{~T}$ (a)

and $3.0 \mathrm{~T}$ (b). Images ( $\mathrm{a}$ and $\mathrm{b}$ ) are

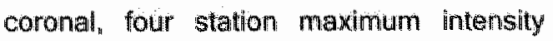
projection images of the peripheral arteries: Note better visibility of distal arteries at 3.0 $T$ (b). Window and level settings were identical for both datasets.

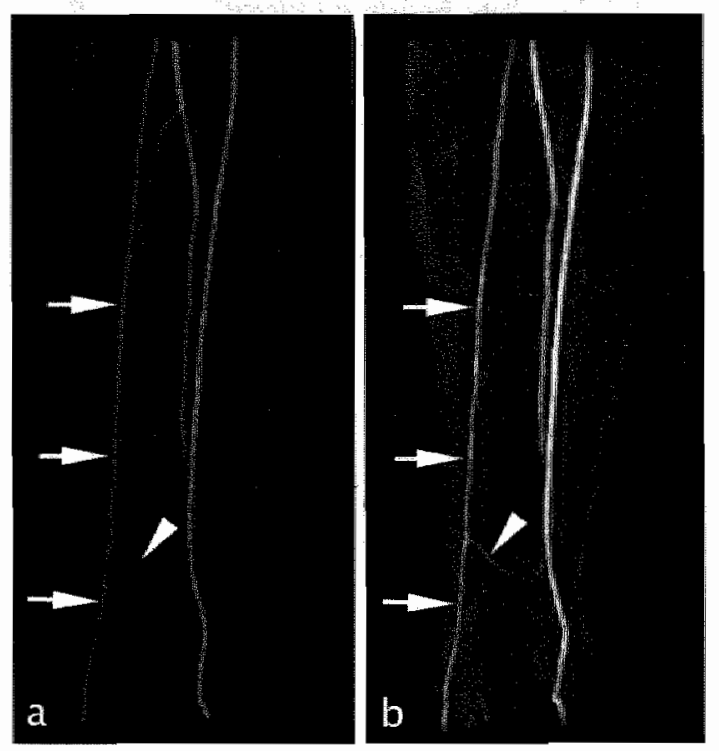

Figure 2

Coronal maximum intensity projection images of distal left lower leg anteries. at $1.5 \mathrm{~T}$ (a) and $3.0 \mathrm{~T}$ (b) in a 29-yearold healthy volunteer. Window and levell settings were identical for both datasets. Note better visibility of distall posterior tibial artery (arrows) and branch thereof (arrowhead) at $3.0 \mathrm{~T}$ (b). 


\section{Discussion}

In this study, we demonstrated the feasibility of imaging peripheral arteries with a system built-in quadrature-body coil at 3.0 T using an imaging and contrast medium injection protocol similar to those used at $1.5 \mathrm{~T}$. To our knowledge, this is the first study demonstrating this.

The image quality obtained in this study at $3.0 \mathrm{~T}$ was equal to or better than state-of-the-art image quality at $1.5 \mathrm{~T}$ for the pelvic and proximal upper leg arteries. For the distal upper leg and lower leg arteries, image quality was considered better at 3.0 T. However, it must be mentioned that SN and $C N$ can be increased at $1.5 \mathrm{~T}$ by using dedicated surface coils. Because such a surface coil was not yet available for the 3.0 T MR system we used, we chose to use the body coil with both systems. It is the expectation that in the near future dedicated coils will also become available for use at 3.0 T and will lead to a further increase in $S N, C N$, and image quality. However, image quality of the lower leg arteries at $3.0 \mathrm{~T}$ was excellent in the current study without the use of such dedicated coils. This can be advantageous because patient positioning will become easier, making the peripheral MR procedure faster and possibly more cost-effective. Formal cost-effectiveness studies that take into account the extra cost of a $3.0 \mathrm{~T}$ magnet with a body coill have to be carried out to substantiate this. Whether image quality without a dedicated coll is sufficient for imaging patients with distal peripheral arterial disease remains to be determined.

It is known from theoretical considerations that imaging at higher fieldstrengths presents several challenges. Due to radio frequency (RF) penetration effects, it can be difficult to achieve good RF field homogeneity in humans at higher field strengths. The result may be that imaging parameters are dependent on location and coill load and thus suboptimal for part of the imaging volume (10). Another challenge is to avoid exceeding specific absorption rate (SAR) limits. At higher imaging frequencies (the Larmor frequency is $127.8 \mathrm{MHz}$ at $3.0 \mathrm{~T}$ vs. $63.9 \mathrm{MHz}$ at 1.5 T), RF energy is absorbed more efficiently which may lead to tissue heating (11). When scanning parameters are kept exactly the same at $1.5 \mathrm{~T}$ and $3.0 \mathrm{~T}$, RF power needed rises quadratically. However, in the current study, choosing the same imaging parameters did not lead to SAR values exceeding safety limits. A recent technical development that may be of benefit to limit the total RF energy delivered while maintaining state-of-the-art scanning protocols is the use of parallel scanning methods such as sensitivity encoding (SENSE). With this scanning method the number of phase encodings can be reduced while maintaining the same spatial resolution. However, the use of SENSE may lead to a substantially lower increase in $S N$ than the theoretically possible factor of 2 , as a direct result of acquiring a smaller number of the samples in a shorter period of time (12).

Because susceptibility increases linearly with field strength, the use of contrast medium injection protocols may have to be changed at $3.0 \mathrm{~T}$ compared with $1.5 \mathrm{~T}$. Due to increased susceptibility, injecting contrast medium at high speeds may lead to signal lloss instead of increased signal intensity (13). We anticipated that the injection rate we routinely use at $1.5 \mathrm{~T}(1.8 \mathrm{~mL} /$ second for the first $15 \mathrm{~mL}$ and $0.6 \mathrm{~mL} /$ second for the remaining $20 \mathrm{~mL}$ ) would be too high and decreased the rate to $1.2 \mathrm{~mL} /$ second and $0.8 \mathrm{~mL} /$ second for the first and second phase, respectively. 
When comparing $S \mathrm{~N}$ and $\mathrm{CN}$ values between $1.5 \mathrm{~T}$ and $3.0 \mathrm{~T}$, the expected factor 2 increase at double the field strength was not the case in the current study for the aortoilliac and proximal upper leg stations. A first possible explanation for this finding may be that the injection rate we employed was too high or that the TE we used was too long. The study by Uematsu et al (14) that examined the feasibility of contrast-enhanced pulmonary MR angiography at $4.0 \mathrm{~T}$, found decreased CN ratios with increasing contrast dose, most notable in the (small) segmental pulmonary arteries. CN increased with a higher contrast dose in the main pulmonary arteries. However, in their study, the injections were done manually and the injection rate was not mentioned. A second explanation may be related to the fact that at $3 T$ coil quality (Q) factors are different than at $1.5 T(15)$. SN is proportional to the square root of $Q$. The body coil we used at 3.0 T has a $Q$-value in empty condition which is almost double the value of the coil used at 1.5 T. However, the effective $Q$-value decreases with higher loads and this effect is more pronounced at $3.0 \mathrm{~T}$. This might partly explain why the gain of SN is lower than expected and different in the two upper stations compared with the two lower stations. A third possible explanation for the lack of increase in $S N$ is that at higher field strengths $T 1$ values increase. Consequently, when almost identical values for TR are used (as was the case in this study), this is expected to lead to a less than twofold increase in signal at $3.0 \mathrm{~T}$. To offset this effect, the TR could be increased. However, increasing the TR to achieve double the SN would increase the duration of the scan or decrease the achievable spatial resolution in a given time. The clinical influence of this effect remains to be determined.

There are theoretical advantages to contrast-enhanced MR angiography at higher field strengths. The most obvious way to improve MR angiography in general is to use the higher $S N$ at $3.0 \mathrm{~T}$ to either increase resolution or to decrease imaging times (by using a shorter TR and/or SENSE) while maintaining the same resolution, or to simply keep the protocol unchanged in regions where SN and $C N$ are currently insufficient. Increasing resolution would be of benefit for imaging the arteries in the lower legs more reliably because current protocols may not be optimal in a substantial number of cases (7). The advantage of reducing imaging scan times is that venous enhancement, that often degrades image interpretability in the lower legs, can potentially be avoided.

In conclusion, contrast-enhanced peripheral MR angiography at $3.0 \mathrm{~T}$ is feasible with an imaging protocol similar to that used at $1.5 \mathrm{~T}$, resulting in substantial increases in the SN and $\mathrm{CN}$ ratios for popliteal and lower leg arteries. For iliac and femoral arteries, there were no large differences between $1.5 \mathrm{~T}$ and $3.0 \mathrm{~T}$ as far as $\mathrm{SN}$ and $\mathrm{CN}$ ratios or image quality was concerned. 


\section{References}

1. Ho K\%, Leirer T, de Haan MW, vani Engelshoven JM. Peripheral MR angiography. Eur Radiol $1999 ; 9: 1765-1774$.

2. Goyen $M$, Debatin JF, Ruehrn SG. Peripheral magnetic resonance angiography. Top Magn Reson Imaging 2001;12:327-335.

3. Nelemans PJ, Leiner $T$, de Vet $H C_{n}$ van Engelshoven JM. Peripheral arterial disease: metaanalysis of the diagnostic performance of MR angiography. Radiology 2000;217:105-11.4.

4. Koelemay MJ, Lijmer JG, Stoker J; Legemate DA, Bossuyt PM. Magnetic resonance angiography for the evaluation of lower extremity arterial disease: a meta-analysis. JAMA 2001:285:1338-1345.

5. Watkins RD; Schenk JF, Rohling KW, et al. Whole body RF coil for 3 Tesla MRI system. In: Proceedings of the 9th Annual Meeting of ISMRM, Glasgow, Scotland, 2001 (abstract 1123).

6. Roberts $S$, Freeman A, Murphy P, Gauss R, Burl M. High definition whole body imaging at 3 Tesla. In: Proceedings of the 9th Annual Meeting of ISMRM, Glasgow, Scotland, 2001 (abstract 2013).

7. Reid SK, Pagan-Marin HR, Menzoian JO, Woodson J, Yucel EK. Contrast-entanced movingtable MR anglography: prospective comparison tô catheter arteriography for treatment planning in peripheral arterial acclusive disease. J Vasc Interv Radiol 2001;12: 45-53.

8. Campeau NG, Huston III J, Bernstein MA, Lin C, Gibbs GF. Magnetic resonance angiography at 3.0 Tesla: initial clinical experience. Top Magn Reson Imaging 2001; 12:183-204.

9. Parker DL, Haacke EM. Signal-to-noise, contrast-to-noise, and resolution. In: Potichen EJ, Haacke EM, Siebert JE, Gottschalk, A, editors. Magnetic resonance angiography: concepts and applications. St. Louis: Mosby; 1993. p 56-79.

10. Bottomley PA, Andrew ER. RF magnetic field penetration, phase shift and power dissipation in biological tissue: implications for NMR imaging. Phys Med Biol 1978;23:630-643.

11. Collins $C M$, Smith $M B$. Calculations of $B(1)$ distribution, SNR, and SAR for a surface coil adjacent to an anatomically-accurate human body model. Magn Reson Med 2001;45:692-699.

12. Pruessmann KP, Weiger M, Scheidegger MB, Boesiger P. SENSE: sensitivity encoding for fast MRI. Magn Reson Med 1999;42:952-962.

13. Kennan RP, Zhong J, Gore JIC. Intravascular suscoptibility contrast mechanisms in tissues. Magn Reson Med 1994;31:9-21.

14. Uematsu $H_{2}$ Dougherty $L$, Takahashi $M_{n}$ et al. Pulmonary MR angiography with contrast agent at 4 Tesla: a preliminary result. Magn Reson Med 2001:46:1028-1030.

15. Vlaardingerbroek MT, den Boer JA. Magnetic resonance imaging: theory and practice. Berlin: Springer Verlag: 1996. p 244-259.614 Leiner et al. 


\section{Chapter 7}

\section{Accuracy of Semi-Automated Analysis of 3D Contrast- Enhanced MR Angiography for Detection and Quantification of Aortoiliac Stenoses}

Investigative Radiology 2005; 40 (8): 495-503

Marianne de Vries, Patrick J. de Koning, Michiel W. de Haan, Alphons G. Kessels, Patricia J. Nelemans, Robbert J. Nijenhuis, R. Nils Planken, G. Boudewijn C. Vasbinder, Jos M.A. van Engelshoven, Rob J. van der Geest, Tim Leiner. 


\section{ABSTRACT}

\section{Purpose}

To compare reproducibility and accuracy of semi-automated stenosis detection and quantification in $3 \mathrm{D}$ contrast-enhanced magnetic resonance angiography (CE-MRA) images with conventional evaluation of 3D CE-MRA in patients with peripheral arterial disease (PAD), using intra-arterial digital subtraction angiography (IA-DSA) as standard of reference.

\section{Materials and Methods}

Twenty-five patients with PAD underwent CE-MRA and IA-DSA. Three blinded observers independently evaluated CE-MRA datasets for stenoses in the aortoiliac region using semi-automated computer analysis. Semi-automated measurements were compared to conventionall measurements of stenosis on CE-MRA datasets, as measured by three other independent abservers blinded to all other measurements. Interobserver agreement was quantified using kappa $(\mathrm{K})$ and intraclass correlation coefficients (ICCs). Sensitivity and specificity were determined for both semiautomated and conventional measurements.

\section{Results}

Semi-automated measurements were successful in 124 of the 125 arterial segments. Sensitivity of semi-automated measurements was $89 \%$ for all observers; specificity varied between $87-89 \%$. For conventional measurements of CE-MRA sensitivity varied between $79-86 \%$; specificity was between $86-96 \%$. There was good interobserver agreement between all readers for semi-automated measurements (combined $k$ for all three observers together $=0.78$; ICC $=0.82$ ), as well as for conventional measurements (combined $k=0.70$; and $I C C=0.83$ ). Differences between ICCs, combined kappa values, and accuracy of both measurements were not significant (all P>.05).

\section{Conclusion}

Semi-automated analysis of aortoiliac 3D CE-MRA has the same high accuracy for detection and quantification of stenoses as conventional readings of CE-MRA. 


\section{Introduction}

Contrast enhanced magnetic resonance angiography (CE-MRA) is increasingly used as an alternative for intra-arterial digital subtraction angiography (IA-DSA) in the diagnostic workup of patients with peripheral arterial disease (PAD). CE-MRA is often preferred to IA-DSA, because it is a minimally invasive and highly accurate technique that gives three-dimensional (3D) information about diseased arteries (13). An important limitation of peripheral CE-MRA, however, remains the limited spatial resolution (4-6).

Analysis of CE-MRA datasets is often performed using the maximum intensity projection (MIP) algorithm in conjunction with viewing of original partitions. A MIP is by definition a 2D image, that selectively displays the voxels with the highest intensity encountered in the projected direction (7). However, the evaluation of 2D MIPs in several directions and original CE-MRA partitions can be a tedious procedure. Moreover, in some cases, MIPs introduce artifacts, resulting in possible overestimation or underestimation of the stenosis severity (7). Using 3D semiautomated stenosis quantification of CE-MRA datasets could possibly overcome these drawbacks.

Recent developments in image post-processing have made it possible to extract and use information present in 3D datasets in more sophisticated ways. For example, semi-automated quantification of arterial lumen diameter and cross sectional area (CSA) from 3D CE-MRA datasets is now possible (8). Several investigators have demonstrated the feasibility of semi-automated stenosis quantification in phantoms and patients with arteriosclerosis (8-12). In most of these patient studies no comparison of semi-automated analysis with an established standard of reference was performed (8-11), except for the study by Frangi et al. in carotid arteries (12). Therefore, to our knowledge, the accuracy of semi-automated measurements for stenosis quantification is currently unknown in patients with PAD.

The purpose of the present study was to compare the reproducibility and accuracy of semi-automated stenosis detection and quantification with conventional evaluation of 3D CE-MRA data in patients with PAD, using IA-DSA as standard of reference.

\section{Material and Methods}

\section{Study Design and Patients}

Twenty-five patients (14 male and 11 female) with suspected PAD, who were referred by their vascular surgeons for CE-MRA, were included in this prospective study. All patients underwent IA-DSA and CE-MRA within a period of 6 weeks. The study protocol was appróved by the local ethics committee. Written informed consent was obtained from all patients before they were included into the study. The mean age of the patients was 65 years (range 51-83). Disease severity was determined according to the Rutherford classification (13). Fifteen patients suffered from 
moderate intermittent claudication (Rutherford grade 2) and 10 patients suffered from severe intermittent claudication (Rutherford grade 3).

Standard of reference was the consensus reading of the IA-DSA images by 3 independent observers, who were blinded for the results of CE-MRA readings. Initially, two observers determined location and degree of stenosis. If these two observers disagreed about the significance of a stenosis ( $<50 \%$ vs. $\geq 50 \%$ ), an adjudication read was performed by a third observer.

\section{CE-MRA Protocol}

All acquilisitions were performed on a $1.5 \mathrm{~T}$ clinical MR scanner (Intera, software release 8.2 , Philips Medical Systems, Best, The Netherlands) with a gradient strength of $30 \mathrm{mT} / \mathrm{m}$ and a rise time of $200 \mu \mathrm{ss}$. To improve signal reception; a quadrature phased-array surface reception coil was used (Philips Medical Systems) (14).

A three-station CE-MRA protocol was performed using flexible image parameters for each station (i.e. aortoiliac, upper and lower limb station) (15). The three-station CE-MRA protocol consisted of four steps: 1) patient positioning, 2) acquisition of low-resolution time-of-flight (TOF) localizer scans 3) acquisition of coronal non contrast-enhanced three-dimensional gradient echo 'mask' scans (which were later subtracted from the corresponding contrast-enhanced images), and 4) acquisition of contrast-enhanced scans using identical imaging parameters as the mask scans.

TOF imaging parameters were TR (ms) / TE (ms) / flip angle ( 9 ) / acquisition time (s): $11 / 6.9 / 50 / 88$. Orthogonal maximum intensity projections (MIPs) of the TOF datasets were generated to prescribe the 3D imaging volume. Using these MIPs, the 3D CE-MRA imaging volume, tailored to individual anatomy of the aortoiliac arteries, was prescribed using a gradient recalled echo sequence (fast field echo, Philips Medical Systems) with the following parameters: repetition time, 4.8 ms; echo time, $1.4 \mathrm{~ms}$; flip angle, $30^{\circ}$; field of view; $470 \times 258 \mathrm{~mm}$ (frequency $\mathrm{x}$ phase); true slice thickness, $2.6 \mathrm{~mm}$ (zero-interpolated to $1.3 \mathrm{~mm}$ ); matrix, $384 \times 156$ (frequency $x$ phase); number of slices, 26-29; acquisition time, 19-22 $\mathrm{s}$. The exact duration of the acquisition varied per patient with the used number of partitions. Overlap between adjacent stations was $3 \mathrm{~cm}$.

Appropriate start of the CE-MRA acquisition was determined using real time bolus monitoring software (BolusTrak, Philips Medical Systems). To depict all three stations $_{\sharp}$ each patient recelved a standard dose of $35 \mathrm{~mL}$ of gadopentetate dimeglumine (Magnevist, Schering, Berlin, Germany) administered at a rate of 1.8 $\mathrm{mL} / \mathrm{s}$ for the first $15 \mathrm{~mL}$, and $0.6 \mathrm{~mL} / \mathrm{s}$ for the remaining $20 \mathrm{~mL}$, followed by a saline flush of $20 \mathrm{~mL}$ at $0.6 \mathrm{~mL} / \mathrm{s}$. All injections were done with a remote controlled $\mathrm{MR}$ compatible injection system (Medrad Spectris, Indianola, PA, USA).

\section{Intra-arterial Digital Subtraction Angiography}

All patients were imaged using Philips Integris V5000 (Philips Medical Systems) digital angiography equipment. In all patients aortic flush arteriograms were obtained 
Using a 5 French pigtaill catheter (Merit Medical Systems Inc., South Jordan, UT) placed in the infrarenal aorta. For the aortoiliac IA-DSA a total of $45-70 \mathrm{~mL}$ contrast medium (lohexal (Omnipaque), $300 \mathrm{mg} / \mathrm{mL}, \mathrm{GE}$ Health, Oslo, Norway) was injected at flow rates ranging from 10 to $15 \mathrm{~mL} / \mathrm{s}$. Arteriograms were obtained in 3 different directions to prevent over-projection; (antero-posterior and 25 degree left and right oblique projections). Additional views were obtained at the discretion of the supervising radiologist.

\section{Image Evaluation}

All observers had multiple years of experience evaluating CE-MRA or IA-DSA datasets. Three observers were radiology fellows and six were radiologists. Image evaluations were independently performed by each observer; observers were blinded to all other results and clinical information. Stenosis quantifications were performed over the entire length of the following 5 arterial segments: aorta (distall 5 $\mathrm{cm}$ ), and both left and right common and external lliac arteries. All 125 vessel segments were analyzed using the semi-automated and conventional approaches. In every vessel segment only the most severe stenosis was recorded. Results of semiautomated and conventional measurements of CE-MRA were compared with $\mid A$ DSA, which was considered as a standard of reference.

The time needed for determining the degree of stenosis for semi-automated and conventional measurements of CE-MRA was recorded.

\section{Semi-automated Stenosis Measurement}

Previous work in phantoms has demonstrated the accuracy of the semi-automated software for assessing and quantifying diameters in CE-MRA datasets using $X$-ray DSA with matched projections as the standard of reference (8). In the present study, three different observers evaluated CE-MRA datasets for presence and degree of stenoses in the aortoiliac arteries of patients with suspected PAD using the same, commercially available software (Medis, Medical Imaging Systems, Leiden, the Netherlands) running on a standard PC workstation (Windows 2000 operating system; Microsoft Inc., Redmond, WA).

Semi-automated analysis required only minimal user interaction and consisted of placement of two markers in the MIP image of the vascular tree. Each vessel segment was analyzed separately. The first marker was placed at the distal boundary, and the second marker was placed at the proximal boundary of the vessel segment. To rule out placement of markers in any high intensity artefacts, the observers checked the position of the markers on 3 orthogonal MIPs. After marker placement the software automatically callculated a path on the original CE-MRA images (without image processing) through the vessel Iumen from the proximal to the distal marker using a fast marching level set method (FMLSM) $(8,16,17)$. The FMLSM stopped when it reached the distal marker; the vessel boundary was determined by signal intensities of the full width $30 \%$ Maximum $(18,19)$. The number of iterations varied according to the length and width of the vessel. The FMLSM ensured that levelsets evolved through the higher signal intensities first, which 
resulted in no significant leakage. Because each vessel segment was analyzed separately no leakage of the propagation front occurred. The detected path was used to initialize a vascular model, which fits the underlying image. The vascular model simulates the course of the vessel as it would be without stenoses (the non-diseased reference vessel). This model is compared with the course of the actual vessel. Subsequently, luminal cross-sectional area (CSA) of the actual vessel and of the reference vessel were determined at one-millimeter intervals in a plane perpendicular to the detected path (Figure 1). Consequently, the degree of stenosis in relation to the non-disease reference vessel was automatically calculated every millimeter using the following formula:

\section{stenosis $\%=(1-$ actual vessel $\mathrm{CSA} /$ reference CSA $) \times 100$}

CSA measurements were then plotted as a function of the position along the centerline. If part of the reference vessel was falsely enlarged due to aneurismal dilatation or due the presence of a vessel branching point, this segment could be manually excluded by the observer. Excluding a dillated part of a vessel segment renders a better fit for the reference vessel model of the "non-diseased" vessel (Figure 2). As a result, the stenosis severity was recomputed automatically at each millimeter interval using these new CSA values of the reference vessel. Dilated parts of vessel segments were excluded if they were more than $10 \%$ wider in comparison with the corresponding contralateral arterial segment.

A stenosis was considered to be hemodynamically significant if the CSA reduction exceeded $50 \%$ (20). Since stenosis computation is based on the original 3D CE-MRA dataset and not on the 2D MIP images neither the resolution of the PC monitor nor window or level settings influenced stenosis computation. Twodimensional MIP images were used to place markers into the artery of interest.

\section{Conventional Stenosis Measurement}

Three additional blinded observers performed conventional evaluations of the $3 \mathrm{D}$ CE-MRA images using a clinical workstation (Easy Vision, Software release 4.1, Philips Medical Systems). Conventional stenosis measurements on 3D CE-MRA images were performed by determining the maximum linear diameter reduction on user defined MIPs, and on original slices of the CE-MRA datasets. For every luminal narrowing, the exact stenosis percentage was recorded. A stenosis was considered to be hemodynamically significant if the linear diameter reduction was equal to or exceeded $50 \%(1-3,21,22)$.

\section{IA-DSA}

Two different observers blinded for all MRA results evaluated IA-DSA datasets for presence and degree of stenoses in the aortoiliac arteries. Stenoses were measured by determining the maximum linear diameter reduction on the antero-posterior or oblique projections. As with conventional 3D-CE MRA evaluation, a stenosis was considered to be hemodynamically significant if the linear diameter reduction 
exceeded $50 \%(3,21,22)$. In case discrepancies existed with respect to the degree of stenosis between the first two observers, a third observer made the final diagnosis. The standard of reference to which the CE-MRA readings were compared was the IA-DSA consensus reading.

\section{Statistical Analysis}

Reproducibility was evaluated by determining interobserver agreement for semiautomated and conventional measurements of CE-MRA, which was calculated using intraclass correlation coefficients (ICCs) and kappa coefficients ( $k$ ). ICCs are a commonly used method to determine variability between different measurements on a continuous scale. This means that ICCs were used to calculate variability between exact stenoses percentages as measured by the different observers (23). Kappa coefficients were calculated after dichotomizing the percentages of stenoses $(0=$ stenosis $<50 \%$, and $1=$ stenosis $\geq 50 \%$ ) (24). Moreover, a combined kappa value for all three observers was computed according to the method of Fleiss (25).

The consensus reading of IA-DSA was used to determine sensitivity and specificity for both semi-automated and conventional measurements. Differences in sensitivity and specificity between semi-automated and conventional measurements of CE-MRA were quantified by constructing receiver operating characteristic (ROC) curves for both methods, and calculating their corresponding areas under the curves (AUCs). The measurement with the highest $A U C$ is the most accurate method for measuring the degree of stenosis.

Five arterial segments were evaluated in each patient. Because all five segments were present in a single dataset and the results of these five measurements are correlated due to biologic and observer dependency, they cannot be regarded as independent observations. Consequently, a bootstrap approach with clustered sampling on patient level was used to test for differences between the AUCs, ICCs and the combined kappa values of the semi-automated and conventional measurements of CE-MRA (26).

$$
\text { P-values below .05 were taken to indicate statistical significance. }
$$

\section{Results}

All CE-MRA and IA-DSA examinations were acquired successfully, and no sideeffects occurred among the 25 patients. Overall, 28 significant lesions (stenosis $\geq$ $50 \%$ ) were identified in 20 patients on IA-DSA in the following arterial segments: 2 in the aorta, 21 in the common iliac arteries, and 5 in the external iliac arteries.

Semi-automated measurements were successful in 124 of the 125 arterial segments. In all but 3 segments the software detected a satisfactory center-lumen line between the two user defined markers.

In 3 arterial segments the software was unable to track a path because there was no signal in part of the vessel segments on CE-MRA due to occlusion, which was consequently recoded as $100 \%$ stenosis $(n=2)$, and because there was no signal owing to in-stent susceptibility artifact $(n=1)$. This segment was excluded from 
analysis; because neither semi-automated nor conventional measurements could evaluate this segment. Four parts of arterial segments had aneurismal dilatations, which were successfully excluded by all 3 observers in the semi-automated analysis. In $37 / 125(30 \%)$ of all vessel segments a small part of the reference vessel was excluded because of the presence of a bifurcation.

In Figure 1 an example is shown of a semi-automated measurement and the corresponding conventional evaluations of CE-MRA and IA-DSA. Use of the semiautomated analysis software resulted in a well-organized presentation of location, degree, area, circumference and extent of the most severe stenosis as extracted from the 3D CE-MRA data:

Figure 1-1e Semi-1eutomated stenosis measurement in right common illac artery
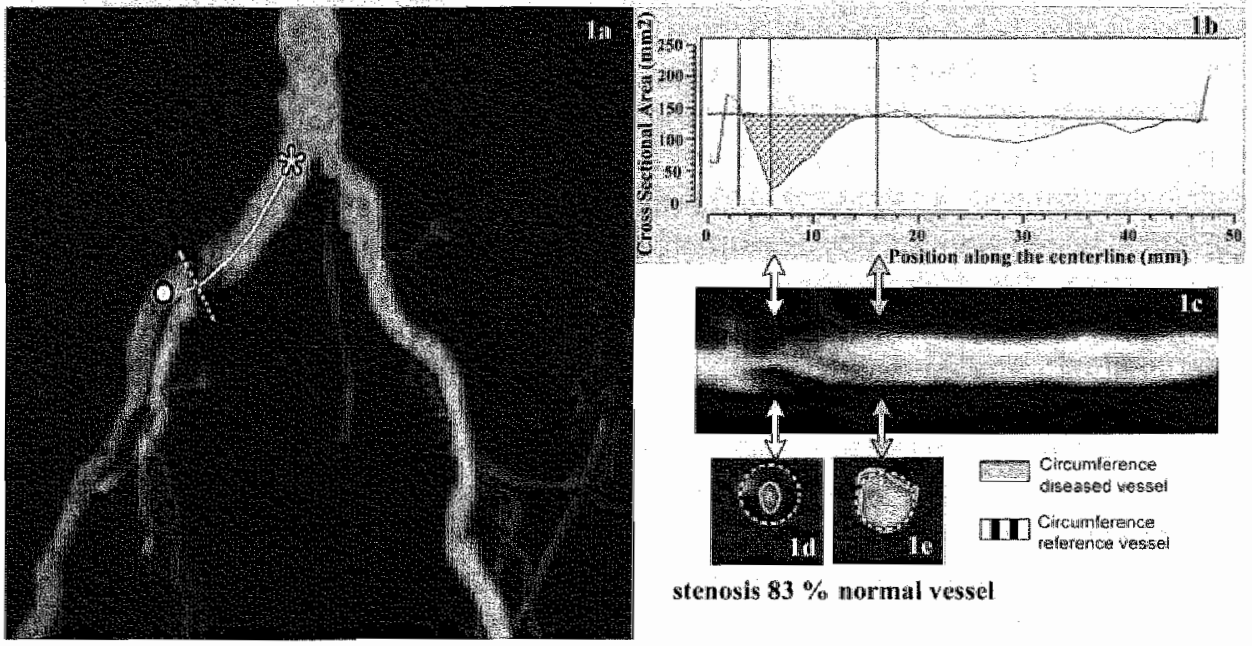

stenosis $83 \%$ mormal vessiet

Subvolume maximum intensity projection (MIP) (a) of the aortoiliac arteries in a 67-year old male patient suffering from intermittent claudication. There are stenoses in both the right common and extemal iliac arteries. After placing the distal marker (circle) and the proximal marker (asterisk), the program automatically determines the path line (white line) between both markers, and the degree anid location of the most severe stenosis (dotted line) in the common iliac artery between both markers. At each millimeter interval the cross sectional area (CSA) (b), the longitudinal reformation (c), and the circumference of the diseased and the reference vessels ( $1 \mathrm{~d}, \mathrm{e}$ ) is calculated starting from the distal marker. 
Figures 1f- Semi-automated stenosis measurement in right extemal ilac artery
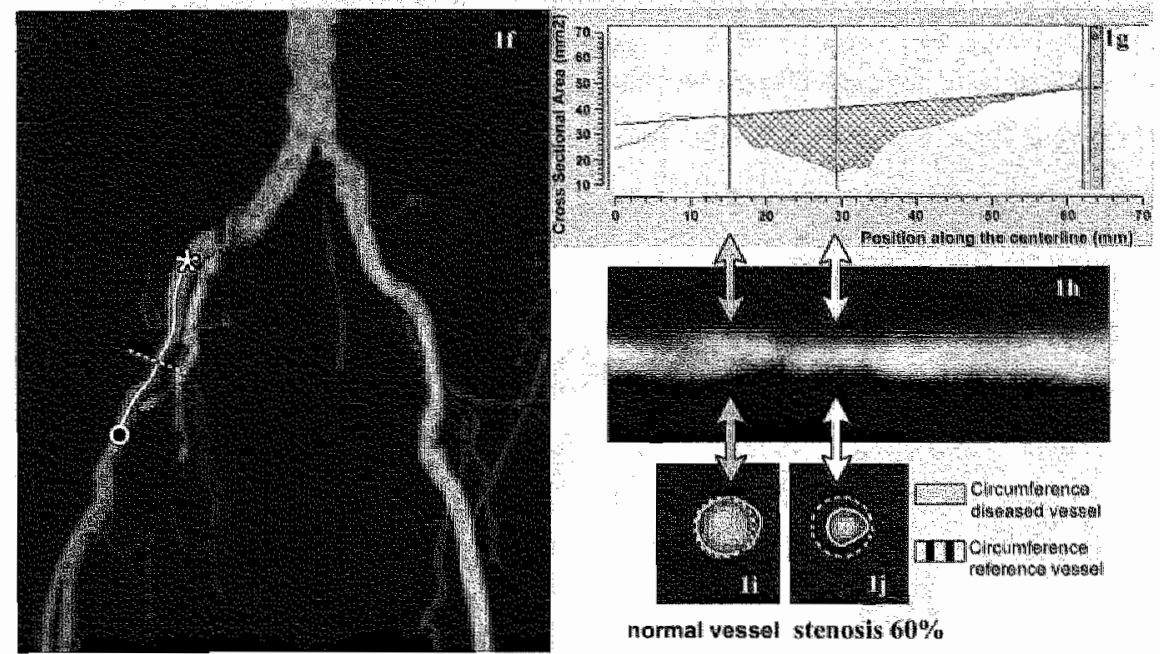

In f-j the results are shown of the semi-automated measurement in the right external iliac artery in the same 67-year old male patient. According to all three observers, the stenosis in the right iliac artery was significant ( $>50 \% \mathrm{CSA}$ reduction). In figure if (continuous white line), and figures $\mathrm{g}-\mathrm{j}$ the location of the stenosis in the external iliac artery is shown. Note the asymmetrical shape of the stenosis (figure 1j).

Figure 1k E-MRA

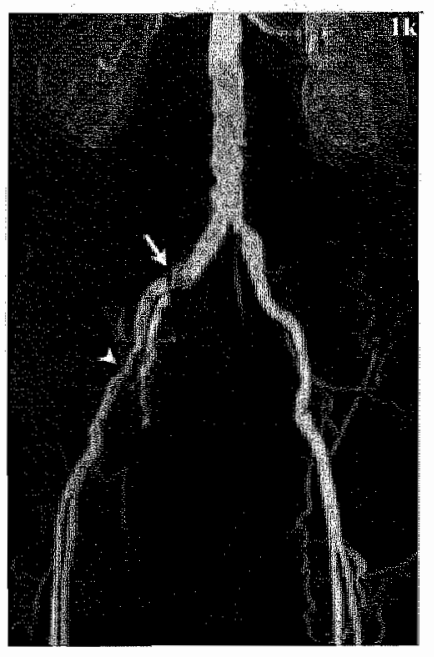

Figure $1 \mathrm{~L}$ IA-DSA

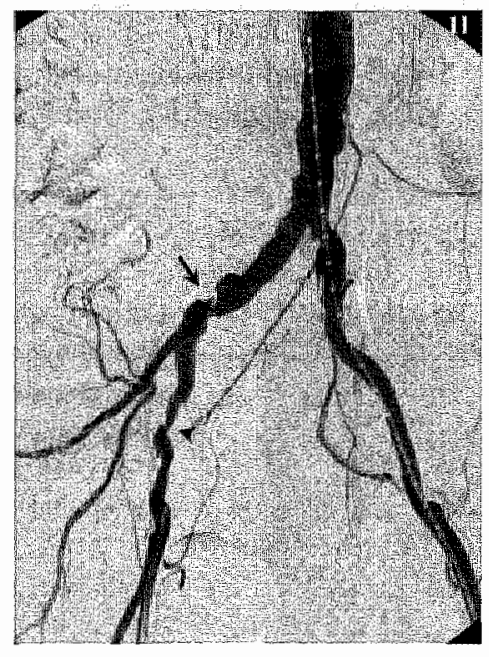

Corresponding whole volume MIP of the CE-MRA ( $k$ ) and IADSA (I) of the aortoiliac arteries in the same 67-year old male patient. Both CEMRA and IA-DSA show significant stenoses lin the right common lilac artery (long arrow). The stenosis in the right external tliac artery (short arrow) was significant according to all three observers of IA-DSA. All three observers of the conventional measurements of CE-MRA failed to recognize the stenosis in right external artery as significant. 
In Figure 2 an example is shown of a better fit of the reference model after excluding an aneurismal part of the vessel segment from the analysis.

Figure 2a-d Semi-automated stenosis measurement in left common lliac artery

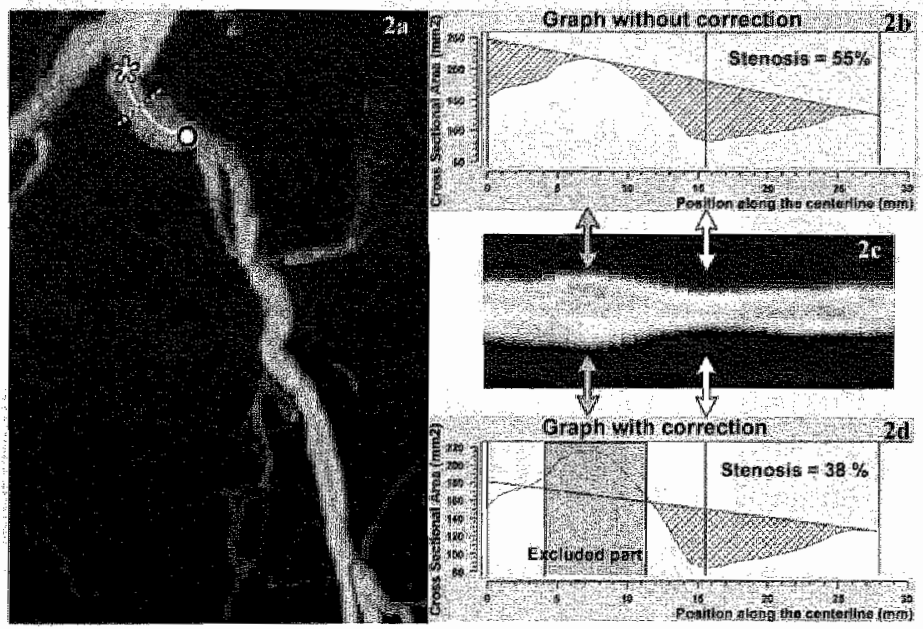

Figure 2a shows a subvolume MP of the aortic bifurcation and left arterial axis of a 80-year old male patient suffering from intermittent claudication.

The dashed line shows the location of the stenosis.

At each millimeter interval the raw cross sectional area (CSA) (2b), the longitudinal reformation (2c), and the corrected CSA (2d) are computed, starting from the distal marker (circle), and ending at the proximal marker (asterisk). The inclined line (marked by the letter $\mathrm{R}$ ) in $2 \mathrm{~b}$ represents the CSA of the reference vessel. The reference vessel is falsely enlarged due to aneurismal dilatation (grey double-headed arrows) of the left common iliac artery. As a result the stenosis severity is overestimated as being $55 \%$. Manual exclusion of the aneurismal part of the artery results in a better fit of the reference line $(R)$, and reduces the degree of stenosis from $55 \%$ to $38 \%$.

Figure 2e CE-MRA Figure 2f IA-DSA
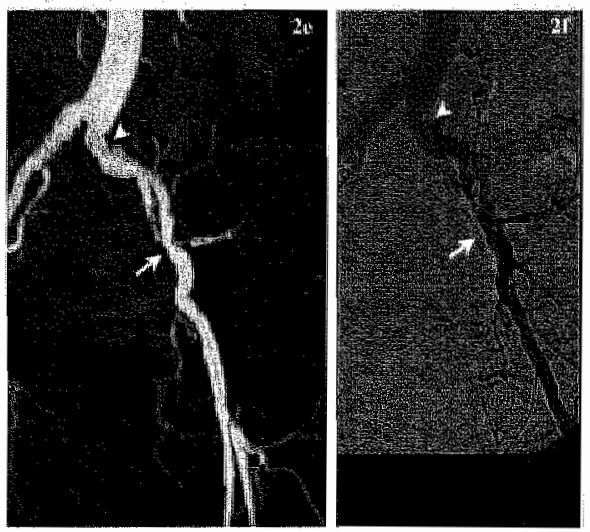

Corresponding whole volume maxímum intensity projection (2e) and IA-DSA (2f) of the aortoilliac arteries in the same patient. Both CEMRA and IA-DSA show non-significant stenosis in the left common iliac artery (long arrow). immediately followed by aneurismal dilatiation. The left common illac artery (small arrow) was evaluated as not significantly stenosed in the opinion of all three observers. The left external iliac artery has a significant stenosis (large arlow). 
Table 1 shows good interobserver agreement for both semi-automated $(\mathrm{ICC}=0.82)$ and conventional measurements of $\mathrm{CE}-\mathrm{MRA}(\mathrm{ICC}=0.83)$. The difference between both ICCs was not significant $(P=.67)$. Higher kappa values were found for semautomated measurements (combined $k=0.78$ ) than for the conventional measurements of CE-MRA (combined $k=0.70$ ) (Table 1), although this difference was not statistically significant $(\mathbb{P}=21)$.

Table 1. Interobserver variation using ICC and $k$-coefficients

\begin{tabular}{|c|c|c|}
\hline & ICC & $k$-coefficients \\
\hline \multicolumn{3}{|l|}{ Semi-automated measurements of CE-MRA } \\
\hline All semi-automated observers 1,2 and 3 & 0.82 & $0.78^{*}(\mathrm{Cl}: 0.68-0.88)$ \\
\hline Semi-automated observer 1 - observer 2 & & 0.84 \\
\hline Semi-automated observer 1 - observer 3 & & 0.73 \\
\hline Semi-automated observer 2 - observer 3 & & 0.76 \\
\hline \multicolumn{3}{|l|}{ Conventional measurements of CE-MRA } \\
\hline All Conventional observers 4,5 and 6 & 0.83 & $0.70^{*}(\mathrm{Cl}: 0.60-0.80)$ \\
\hline Conventional observer 4 -observer 5 & & 0.68 \\
\hline Conventional observer 4 -observer 6 & & 0.78 \\
\hline Conventional observer 5 -observer 6 & & 0.64 \\
\hline
\end{tabular}

* Combined $k$-coefficients $=\kappa$-value for all three abservers together; $\mathrm{Cl}=95 \%$ confidence interval.

Table 2 Diagnostic accuracy of semi-automated and conventional evaluation of CE-MRA

\begin{tabular}{|c|c|c|c|c|c|c|}
\hline & \multicolumn{2}{|c|}{ Sensitivity $(95 \% \mathrm{Cl})$} & \multicolumn{3}{|c|}{ Specifificity (95\%Cl) } & \multirow[t]{2}{*}{ AUC } \\
\hline \multicolumn{6}{|l|}{ Semi-automated CE-MRA } & \\
\hline Semi-automated abs 1 & $25 / 28 \quad 89 \%$ & $(72 \%-98 \%)$ & $83 / 95$ & $87 \%$ & $(81 \%-94 \%)$ & 0.94 \\
\hline Semi-automated obs 2 & $25 / 28 \quad 89 \%$ & $(72 \%-98 \%)$ & $85 / 95$ & $89 \%$ & $(83 \%-96 \%)$ & 0.94 \\
\hline Semi-automated obs 3 & $25 / 28 \quad 89 \%$ & $(72 \%-98 \%)$ & $85 / 95$ & $89 \%$ & $(83 \%-96 \%)$ & 0.95 \\
\hline \multicolumn{7}{|l|}{ Conventional| CE-MRA } \\
\hline Conventionall obs 4 & $24 / 28 \quad 86 \%$ & $(67 \%-96 \%)$ & $89 / 95$ & $94 \%$ & $(89 \% \times 99 \%)$ & 0.96 \\
\hline Conventionall obs 5 & $22 / 2879 \%$ & $(63 \%-94 \%)$ & $94 / 95$ & $96 \%$ & $(90 \%-99 \%)$ & 0.97 \\
\hline Conventionali obs 6 & $24 / 28 \quad 86 \%$ & $(67 \%-96 \%)$ & $82 / 95$ & $86 \%$ & $(78 \%-93 \%)$ & 0.95 \\
\hline
\end{tabular}

The actual number of stenoses and arterial segments are listed. Obs= observer, AUC: area under the ROC curve. Cl: $95 \%$ confidence interval. The $\|$ A-DSA was used as standard of reference. Note: one arterial segment was not depicted by IA-DSA, and one arterial segment was excluded from CE-MRA due to the presence of a stent. Therefore 1,23 arterial segments remained for analysis.

In Table 2, sensitivity, specificity and areas under the ROC curve are listed for each observer. Both conventional and semi-automated measurements by all observers had good results regarding sensitivity and specificity. Comparing semi-automated with conventional measurements of CE-MRA resulted in minor differences in sensitivity and specificity between both measurements for all six observers. Likewise, 
the AUCs based on semi-automated and conventional measurements of CE-MRA showed no statistically significant differences (all $P>.05$; Figure 3 ). The 2 occlusions were correctly detected as $100 \%$ stenosis by all observers with the semi-automated and convertional measurements.

Figure 3 ROC curve of semi-automated and conventional measurements

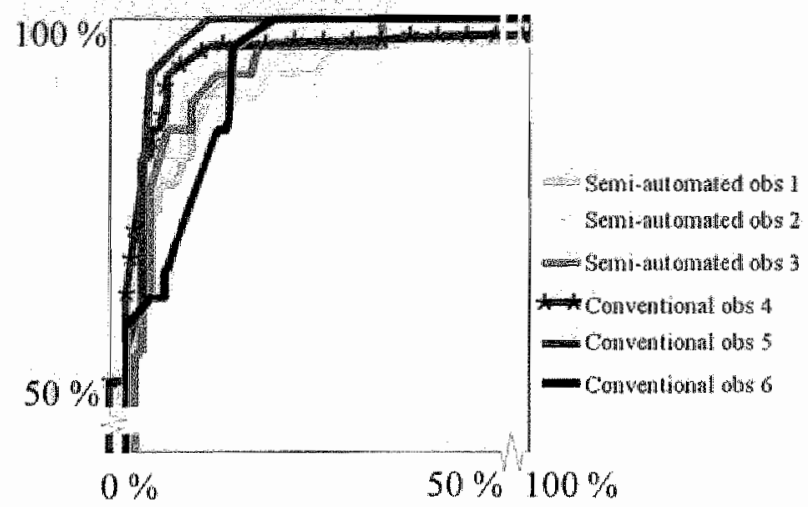

Obs $=$ observer

\section{Discussion}

The present study demonstrates that semi-automated evaluation of 3D CE-MRA datasets is as good as conventional measurements to determine degree of stenosis. Both semi-automated and conventional measurements of CE-MRA have the same high accuracy, and have the same good interobserver agreement according to the ICCs. As such, our results were in accordance with the literature (1). Semiautomated 3D analysis has the additional advantage that it yields a complete description of vascular morphology. For instance, next to stenosis quantification, vessel diameter and CSA could easily be quantified along the entire artery.

Semi-automated measurements are easy to conduct with minimal observer interaction needed (placement of two markers in the vessel) to assess stenosis severity. In addition, observers have the means to correct the analysis interactively. For example, when a part in the reference model of the vessel is falsely enlarged due to aneurysm or the presence of a branching point, the observer can exclude this part of the vessel from the analysis (Figure 2). Exclusion of such parts of the vessell is a substantial improvement over earlier studies, because it allows semi-automated measurement of stenoses in or around bifurcations or aneurismal dilatations, whereas this was a limitation in another study (10).

Although the method used in the current study is somewhat different when compared to the method presented by van Bemmel et al (11), both studies 
underscore that determination of reference vessel diameter can be performed in an easy and robust fashion. This is of importance since stenoses are frequently situated close to or in these segments. Another improvement is that semi-automated measurements automatically determine stenosis severity. In other studies the observer (10-12) needed to mark the healthy reference vessel for every stenosis. In our method such actions are fully automated, with the exception that the observer has to verify whether the course of the reference vessel model represents the course of the vessel as it would be without stenoses.

Interobserver agreement in terms of ICCs suggests that semi-automated measurements are as good as conventional measurements of CE-MRA, whereas in terms of kappa values semi-automated measurements appear to be better. An explanation for this finding is that with semi-automated measurements even minor degrees of luminal narrowing are reported as stenoses (albeit with a low stenosis percentage). With conventional measurements these minor stenoses are often scored as no stenosis at all (i.e. $0 \%$ ). This results in an artificial increase of the $1 \mathrm{CC}$ for conventional measurements, since $I C C$ is based on comparing exact stenosis percentages. However, with the use of $k$-coefficients these minor stenoses are all grouped into the category "no significant stenosis at all", so small variations between observers have no impact on the measure of interobserver agreement. In our opinion the use of $\mathrm{k}$-coefficients corresponds better to clinical practice where treatment decisions are also based on classification of stenosis severity greater or smaller than $50 \%$ lumen reduction.

The higher kappa values of the semi-automated measurements compared to conventional measurements are most likely explained by the minimal user interaction needed for semi-automated stenosis measurements. This is in contrast to conventional measurements, which are for several reasons susceptible to variations between operators. First, selection of a healthy segment and the segment with maximum diameter reduction of a vessel is user dependent. Second, if window and level settings are not standardized (19), the choice of window and level setting to display the dataset can vary between observers, which can also induce differences in measurements of stenosis severity. However, although interobserver agreement was higher, it was not perfect for the semi-automated measurements, because each observer excluded slightly different parts of the reference vessel. As a result, the size of the reference vessel slightly differs in various analyses of the same vessel, resulting in differences in degree of stenosis severity.

The semi-automated measurements' use of the CSA for stenosis quantification improves the insight into the geometrical shape of the stenosis, as compared to the use of conventional linear diameter measurements. However, determination of the CSA is a different approach and is at present not widely used due to inappropriate image analysis software. Nevertheless, we have demonstrated that both stenosis quantification based on CSA (for the semi-automated measurements) and diameter reduction (for the conventional measurements) have the same high accuracy. These findings correspond with a study of Wise et al., who reported equally high accuracies of carotid stenosis quantification based on both CSA and diameter reduction (20). The explanation for this finding is that, in contrast to the general assumption, ex vivo measurements of stenoses demonstrate that the 
lumen in stenoses have a wide variely of geometric shapes and are rarely circular or of 'hour glass' shape (27). This means that $50 \%$ linear diameter reduction does not always result in $75 \%$ CSA reduction. Future studies are necessary to compare CSA measurements of CE-MRA to CSA measurements of IA-DSA datasets with the recently introduced rotational $3 D$ DA-DSA.

The present study has several limitations. The accuracy of semi-automated measurements of CE-MRA was determined only for the aortoiliac arteries, since most patients with PAD receive interventional treatment for significant stenoses in the aortoiliac region. Future studies are necessary to also determine the accuracy of semi-automated measurements of CE-MRA for the arteries in the upper and lower legs.

In the current study, semi-automated measurement of degree of stenosis could be performed in about $1-1 / 1 / 2$ minutes per vessel segment. The most time consuming actions were loading the data from CD-ROM (20-40 seconds), detecting the pathline (20-30 seconds) and subsequent verification and sometimes editing of the segmentation (10-60 seconds). To reduce time it is already been possible to analyse multiple arterial segments at once. It is likely that the duration of the semiautomated analysis can be shortened considerably in the future by optimising the algorithm in terms of random access computer memory efficiency and the required user interaction for correction of segmentation errors, for instance around bifurcations.

A limitation of the semi-automated software is that path tracking is impossible in case of an occlusion of the vessel. However, since occlusions can easily be identified visually, this problem can be overcome by placing the markers in a manner that exclude the occlusion from analysis.

How can the results of the current study be used in clinical practice? The current study has shown that semi-automated stenosis measurements of CE-MRA datasets are equally accurate when compared to conventional evaluation. In the future it is likely that more widespread utilization of semi-automated analysis will enable faster quantification of stenoses on CE-MRA for patients with PAD, thereby replacing conventional measurements of CE-MRA, or helping (in)experienced readers to verify their conventional measurements of CE-MRA. Another useful application of semi-automated evaluation of stenoses would standardization of stenosis measurement in (multicenter) clinical trials. In addition, accurate quantification of the shape of the vessel might be helpful for the exact determination of size of vascular stents and endoprostheses prior to intervention.

According to Krupinski, radiologists will probably be using computer-aideddetection software as an everyday tool to detect various diseases in the future (28). For example, in other fields such as mammography, high accuracy has been reported in a preliminary study for computer-aided-detection of breast cancer (29). In other studies, Jiang and Warren Burhenne demonstrated that radiologists were able to detect lesions more readily on mammography with the use of computer-aideddetection software. A salient finding was that even the most experienced readers benefited on occasion from having computer-aided-detection input during image interpretation process $(30,31)$. Our study showed that another promising application for computer-aided-detection is determination of degree of stenosis in patients with PAD. 
In conclusion, semi-automated evaluation of aortollac 3D CE-MRA has the same high accuracy for significant stenosis detection and quantification as conventional readings of CE-MRA. In the future, semi-automated measurements may replace or can be combined with conventional measurements of CEMRA. In addition, accurate quantification of vessel diameter might be suitable for determination of exact size of vascular stents and endoprostheses prior to intervention. 


\section{References}

1. Nelemans PJ, Leiner T, de Vet HC, van Engelshoven JM. Peripheral arterial disease: metaanalysis of the diagnostic performance of MR angiography. Radiology 2000;217(1):105-114.

2. Koelemay MJ. Lijmer JG, stoker J, Legemate DA, Bossuyt PM. Magnetic resonance angiography for the evaluation of llower extremity anterial disease: a meta-analysis. Jama 2001:285(10):1338-1345.

3. Leiner $T$, Kessels $A G$, Schurink $G W_{\text {n }}$ et al. Comparison of contrast-enhanced magnetic resonance angiography and digital subtraction angiography in patients with chronic critical ischemia and tissue loss. Invest Radiol 2004;39/7):435-444.

4. Ho KY, Leiner T, de Haan MW, van Engelshoven JM. Peripheral MR angiography. Eur Radiol 1999;9(9):1765-1774.

5. Hood MN, Ho VB, Foo TK, Marcos HB, Hess SL, Choyke PL. High-resolution gadoliniumenhanced 3D MRA of the infrapopliteal arteries. Lessons for improving bolus-chase peripheral MRA(1). Magn Reson Imaging 2002;20(7):543-549.

6. Turski $P$. Sources of Variability in Measuring Carotid Stenosis on Time-Resolved ContrastEnhanced MR Angiograms. AJNR Am J Neuroradiol 2002;23(2):178-179.

7. Anderson CM, Saloner D, Tsuruda JS, Shapeero LG, Lee RE. Artifacts in maximum-intensityprojection display of MR angiograms. A.JR Am J Roentgenol 1990; 154(3):623-629.

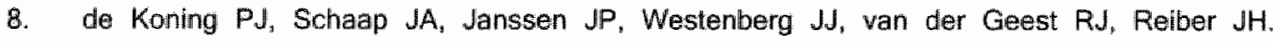
Automated segmentation and analysis of vascular structures in magnetic resonance angiographic images. Magn Reson Med 2003;50(6):1189-1198.

9. Boskamp $T$, Rinck D, Link $F$, Kummerlen $B$, Stamm $G$, Mildenberger $P$. New vessel analysis tool for morphometric quantification and visualization of vessels in $C T$ and MR imaging data sets. Radiographics 2004;24(1):287-297.

10. Hernandez-Hoyos $M$, Orkisz $M$, Puech $P$, Mansard-Desbleds $C$, Douek $P$, Magnin IE. Computer-assisted analysis of three-dimensional MR angiograms. Radiographics $2002 ; 22(2): 421-436$.

11. van Bemmel $C M$, Elgersma $O E$, Vonken $E J_{\text {, Fiorelli } M} M_{*}$ van Leeuwen MS, Niessen WJ. Evaluation of semiautomated internal carotid artery stenosis quantification from 3-dimensional contrast-enhanced magnetic resonance angiograms. Invest Radiol 2004;39(7):418-426.

12. Frangl AF. Niessen W., Nederkoorn PJ, Bakker J, Mali WP, Viergever MA. Quentitative analysis of vascular morphology from 3D MR angiograms: In vitro and in vivo results. Magn Reson Med 2001;45(2):311-322.

13. Rutherford $R B$, Baker JD, Ernst $C$, et al. Recommended standards for reports dealing with iower extremity ischemia: revised version. J Vasc Surg 1997;26(3):517-538.

14. Leiner $T$, Nijenhuis $R J$, Maki JH, Lemaire $E$, Hoogeveen $R$, van Engelshoven JM. Use of a three-station phased array coil to improve peripheral contrast-enhanced magnetic resonance angiography. J Magn Reson Imaging 2004;20(3):417-425.

15. Leiner $T$, Ho KY, Nelemans $P J$, de Haan MW, van Engelshoven JM. Three-dimensional contrast-enhanced mowing-bed infusion-tracking (MoBl-track) peripheral MR angiography with flexible choice of imaging parameters for each field of view. J Magn Reson Imaging 2000;11(4):368-377.

16. Sethian JA. A fest marching level set method for monotonically advancing fronts. Proc Nat Acad Sci U SA 1996;93(4):1591-1595. 
17. Janssen $J P$, Koning $G$, de Koning $P J$, Tuinernburg $J C$, Reiber $J H$. Validation of a new method for the detection of pathlines in vascular $x$-ray images. Invest Radiol 2004;39(9):524:530.

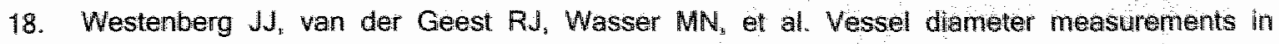
gadolinium contrast-enhanced three-dimensional MRA of peripheral arteries. Magn Reson limaging $2000 ; 18(1): 13-22$.

19. Hoogeveen RM, Bakker C.J, Viergever MA. Limits to the accuracy of vessel diameter measurement in MR angiography. J Magn Reson Imaging 1998;8(6):1228-1235.

20. Wise SW, Hopper KD, Ten Have T, Schwartz T. Measuring carotid artery stenosis using CT angiography: the dilemma of artifactual lumen eccentricity. AJR Am J Roentgenol 1998; 170(4):919-923.

21. Meaney JF, Ridgway JP, Chakraverty $S$, al. Stepping-table gadolinium-enhanced digital subtraction MR angiography of the aorta and lower extremity arteries: preliminary experience. Radiology 1999;211(1);59-67.

22. Rofsky $N_{4}$ Adelman MA. MR angiography in the evaluation of atherosclerotic peripheral vascular disease. Radiology 2000;214(2):325-338.

23. Shrout PE, Fleiss JL. Intraclass Correlations: Uses in Assessing Rater Reliability. Pshychological Bulletin 1979;86(2):420-428

24. Cohen J. Weighted kappa: nominal scale agreement with provision for scaled disagreement or partial credit. Psycholl Bull 1968;70:213-230.

25. Fleiss JL. Statistical Methods for raters and proportions.: $2 d$ ed. New York: John Wiley \&Sons; 1981. 325-332 p.

26. Rutter CM. Bootstrap estimation of diagnostic accuracy with patient-clustered data. Acad Radiol $2000 ; 7(6): 413-419$.

27. Yuan $\mathrm{C}$. Kerwin WS. MRI of atherosclerosis. J Magn Reson Imaging 2004;19(6):710-719.

28. Krupinski EA. Computer-aided Detection in Clinical Environment: Benefits and Challenges for Radiologists. Radiology 2004;231(1):7-9.

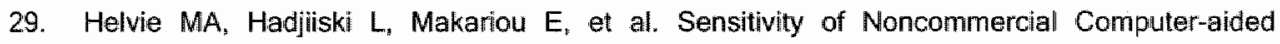
Detection System for Mammographic Breast Cancer Detection: Pilot Clinical Trial. Radiology 2004;231(1):208-214.

30. Jiang $Y$, Nishikawa RM, Schmidt RA, Metz CE, Giger ML. Doi $K$. Improving breast cancer diagnosis with computer-aided diagnosis. Acad Radiol 1999;6(1):22-33.

31. Warren Burnenne $\mathrm{LJ}$, Wood SA, D'Orsi $\mathrm{CJ}$, et al. Potential contribution of computer-aided detection to the sensitivity of screening mammography. Radiology $2000 ; 215(2): 554-562$. 


\section{Chapter 8}

General discussion 


\section{GENERAL DISCUSSION}

Several studies demonstrated that imaging of patients with peripheral arterial disease (PAD) to determine the optimal revascularization strategy can be performed accurately using either duplex ultrasound (DUS), contrast-enhanced MR angiography (CE-MRA), or computed tomographic angilography (CTA) (1-4). However, it is difficult to translate the high accuracy obtained by DUS, CE-MRA, or CTA into a pragmatic clinical approach. Since it remains uncertain which imaging modality is optimal for treatment planning and, at the same time, which is the most cost-effective. This thesis sought to find the optimal initial imaging modality on the basis of the results of the Diagnostic Imaging for Peripheral Arterial Disease (DIPAD) study, which is a randomized cost-effectiveness study.

\section{DIPAD study}

The DIPAD study was performed at four Dutch hospitals: Maastricht University Medical Center, University Medical Center St Radboud Nijmegen "Erasmus Medical Center Rotterdam, and one general hospital St Catharina Hospital Eindhoven. In this prospective multicenter study, all patients with PAD who needed an imaging workup for the assessment of the optimal treatment strategy were randomly assigned to $C E$ MRA or the currently employed test. The currently employed tests were CTA in Rotterdam and DUS in the other three participating centers. Thus patients in Rotterdam were randomized to CE-MRA or CTA, and patients in Maastricht, Eindhoven, and Nijmegen were randomized to CE-MRA or DUS.

\section{Improving imaging protocol}

To perform the best possible cost-effectiveness study, it is important to use up-todate imaging protocols, especially for the CE-MRA $(5,6)$.

Chapter 2 describes the additional value of using a parallel imaging technique (SENSE) for optimizing the CE-MRA protocol. This technique (SENSE) reduces the scan time by simultaneously collecting MR signals from multiple reception coils $(7,8)$. Despite the reduction of scan time (from 132 seconds in the control group to 80 seconds with SENSE), we were unable to avoid all venous enhancement in the lower legs hampering image interpretation. To avoid venous enhancement, the imaging times would have to be accelerated to $5-6$ seconds per station, resulting in a major signal drop even when SENSE is used (9). When a distal bypass is considered, it is particularly important to depict the arteries of the lower leg accurately. Several studies showed that it is easier to avoid venous enhancement in such cases by depicting the lower leg arteries first, and subsequently the aortoiliac and upper leg arteries with an additional contrast bolus (i.e., hybrid CE-MRA protocol) $(10,11)$. During our cost-effectiveness study, we did not use a hybrid CE-MRA protocol, although we recently implemented a hybrid CE-MRA protocol for patients with critical ischemia. Nonetheless, our study shows that CE-MRA using SENSE did improve temporal and spatial resolution and enlarged the anatomical coverage. We conclude that SENSE facilitates routine " high-quality depiction of the entire peripheral vascular 
tree of the legs, including the pedal arch. Subsequently, this MR protocol using SENSE was implemented into the cost-effectiveness study.

\section{Outcome measurements}

Treatment of PAD is usually evaluated in terms of clinical parameters such as the Rutherford classification and the ankle brachial pressure index (ABPI). A major drawback of these clinical parameters is that they poorly reflect the perspective of the patient after treatment (12-14). As a result, several studies propose measuring quality of life (QOL) as a primary endpoint in evaluating treatment effects (15-19).

Quality of life can be assessed with generic questionnairres and/or diseasespecific questionnaires. Whether disease-specific or generic questionnalres most accurately determine the quality of life of patients with PAD remains unclear. Therefore, we compared the ability of disease-specific and generic questionnaires to assess quality of life at baseline and to detect changes after treatment in patients with PAD. As Chapter 3 shows, the disease-specific questionnaire VascuQol was preferred for detecting changes in disease severity over the generic questionnaires (SF-36 and EuroQol-5D). Therefore, we used quality of life as measured by the VascuQol as an important outcome measure in the cost-effectiveness study.

The VascuQol is only one of several disease-specific questionnaires (e.g., CLAU-S) $(20,21)$ that, can assess the quality of life of patients with PAD. However, we chose to implement the VascuQol in the cost-effectiveness study, since the VascuQol is the only disease-specific questionnaire that measures the quality of life of claudication patients as well as critical ischemia patients.

\section{Choosing the best imaging work-up}

The cost-effectiveness study shows that the CE-MRA increases the physician's confidence in taking treatment decisions, which reduces the need for additional imaging compared to that of DUS. On the other hand DUS was associated with lower total diagnostic costs compared to CE-MRA, due to the high investment costs of the MR scanner.

When we compared DUS and CE-MRA with CTA, we found that CTA led to cost savings compared to both MRA and DUS. Moreover, both CTA and CE-MRA required less additional vascular imaging tests and increased the degree of physician's confidence for taking treatment decisions than DUS. However, CTA requires radiation and a nephrotoxic contrast agent, which can be harmful to the patient (22).

Which imaging test is the best? Our study shows that CE-MRA, DUS, and CTA performed equally well in the initial imaging workup for patients with PAD, albeit the three imaging modalities had different qualities that counted in their favor. Therefore, the answer to this question will depend on local expertise with these techniques and cost considerations relevant to the specific hospital setting.

Expertise is very important. DUS gives good results in terms of sensitivity and specificity, even regarding depiction of the lower leg arteries, provided that the ultrasonographers are highly experienced (23-25). Similarly, the image quality of 
MRA depends on the quality of the equipment and experience of the MR technologists $(5,6)$. Experience is important for the evaluation of CTA images due to the presence of vessel wall calcifications, which may obscure stenoses and obstructions.

The treatment policy of a Dutch hospital depends not only on costs, but aiso on clinical efficiency. In this study, CTA was the least expensive modality and required $65 \%$ fewer additional imaging tests than DUS. Should we therefore recommend CTA? CTA is recommended if the radiology department in question, is experienced in performing peripheral CTA, provided that the patient has no renal insufficiency. Centers that seek to improve their Imaging workup might opt to expand their expertise in CE-MRA (rather than DUS or CTA), as the clinical value of CE-MRA outweighs that of DUS, and the patient is not exposed to nephrotoxic contrast agents and radiation.

Critical ischemia patients often undergo distal bypass surgery, for which optimal depiction of the lower leg arteries is essential. In our study, CTA and CEMRA required fewer additional imaging in critical ischemia patients than DUS. Future studies may show whether CE-MRA is to be preferred over CTA, as CE-MRA can depict the lower leg arteries accurately even in patients with criticall ischemia $(1,10,26,27)$. The role of CTA for depicting the lower leg arteries in critical ischemia patients is still controversial. For example, Catalano et al. found that it was possible (4), whereas other studies did not find CTA to be an accurate technique for depicting the lower leg arteries $(28,29)$.

Consistent with Janser et al. (30), the total costs were primarily influenced by the costs of therapy and possible complications after therapy (Chapter 4), while the cost of diagnostic imaging had only a marginal impact on the amount of the total cost. From this perspective, CE-MRA can be recommended, since the detrimental higher diagnostic costs of CE-MRA in comparison to those of DUS were found to be negligible as compared to the costs associated with therapeutic interventions, and consequently to the amount of the total cost (Chapter 4).

Can we generalize our results to all other hospitals? The DIPAD study was a multicenter trial, and in each hospital we found different salaries, different equipment costs, different diagnostic and therapeutic costs. Moreover, a limitation of our study was that each hospital used a different CE-MRA protocol. Nevertheless, a subgroup analysis showed no significant differences in the total additional imaging costs, total diagnostic costs, and total costs of DUS and CE-MRA among the participating hospitals. Therefore, it seems that the results of the DIPAD study can be generalized to all other hospitals.

\section{Future directions for CE-MRA}

Using dedicated coils and up-to-date high-resolution peripheral CE-MRA protocols are particularly important for patients with critical ischemia. Optimal spatial resolution of the lower leg arteries is necessary since these patients often undergo distal bypasses. Optimal temporal resolution is also essential, because critical ischemia patients often have disturbing venous enhancement projecting over the lower leg arteries. Dedicated coils, such as a peripheral vascular coil or a body coill used for 
the lower legs, are necessary for this purpose, especially when a parallel imaging technique is combined $(31,32)$. Using 1.0 molar contrast agents may also help improve signal-to-noise ratio (33).

The use of a 3.0 Tesla MR scanner can improve the signal-to-noise ratio for the lower leg arteries, as is described in Chapter 6 . A limitation of the 3.0 Tesla is that the field of view is smaller compared to the 1.5 Tesla. Therefore, the acquisition time for depicting the entire peripheral vascular tree with the 3.0 Tesla is increased than with 1.5 Tesla. Nevertheless, we found that the role of the 3.0 Tesla seems to be very promising for peripheral CE-MRA. Spatial resolution and signal-to-noise ratio can be further improved when dedicated coils become avallable for the 3.0 Tesla.

A disadvantage of peripheral CE-MRA is that the evaluation of the images is time consuming and therefore expensive, but software that successfully detects the stenosis of an artery and assesses its severity in a semi-automated way, as described in Chapter 7, could improve this situation. This software can be applied to standardize stenosis measurements in (multicenter) clinical trials or in everyday clinical practice. Future studies are necessary to determine whether the software is also useful for detecting stenosis of the upper and lower legs, and whether the software can predict the exact size of vascular stents and endoprostheses prior to intervention.

\section{Future directions of CTA}

The introduction of multidetector CT enables faster acquisition, thereby improving spatial resolution and allowing larger anatomical coverage (4). Newer multi-detector CT scanners (such as the 8,16 , or 32 slice) increase the acquisition speed even more, which reduces the amount of potentially nephrotoxic contrast agent. Furthermore, it might be possible to reduce the radiation dose by optimizing the acquisition protocol as has been done with the protocoll for the multi-detector $\mathrm{CT}$ used in other parts of the body (34).

New post-processing techniques such as a bone-image removal method might be useful in improving image evaluation, since arteries are often obscured by bone, and vessel wall calcifications. A bone-image removal method makes use of an additional, non-enhanced CT scan to create a mask of the bone and if present the vessel wall calcifications, that is used to remove the bone and wall calcifications from the image of the contrast-enhanced CT scan (35). However, the limitation of increased radiation dosages must be overcome to make this useful technique for imaging abdominal arteries.

\section{Conclusions}

The choice of DUS, CE-MRA, or CTA for the initial Imaging workup for patients with PAD depends on the local expertise with these techniques and on cost considerations relevant to the local hospital setting. Centers that seek to improve their imaging workup might opt to expand their expertise in CE-MRA rather than DUS or CTA, as the clinical value of CE-MRA outweighs DUS and no nephrotoxic contrast agent is necessary. Moreover, we recommend to be creative with dedicated 
colls and parallel imaging techniques to improve the CE-MRA protocol. Future use of semi-automated stenosis detection systems and/or by higher magnetic field strengths (3.0 T) might further improve CE-MRA. 


\section{References}

1. Koelemay MJ, Lijmer JG, Stoker $J$, Legemate DA, Bossuyt PM. Magnetic resonance angiography for the evaluation of lower extremity arterial disease: a meta-analysis. Jama $2001,285(10): 1338-1345$

2. Nelemans PJ, Leiner T, de Vet HC, van Engelshoven JM. Peripheral arterial disease: metaanalysis of the diagnostic performance of MR angiography. Radiology 2000;217(1):105-114.

3. Visser K, Hunink MG. Peripheral arterial disease: gadolinium-enhanced MR angiography versus color-guided duplex US-a meta-analysis. Radiology 2000;216(1):67-77.

4. Catalano $C_{n}$ Fraioli $F$, Laghi $A$, et al. Infrarenal aortic and lower-extremity arterial disease: diagnostic performance of multi-detector row CT angiography. Radiology $2004 ; 231(2): 555-563$.

5. Swan JS, Kennell TW, Acher $C W$, et al. Magnetic resonance angiography of aorto-iliac disease. Am J Surg 2000;180(1):6-12.

6. Eiberg JP, Lundorf $E$, Thomsen $C$, Schroeder TV. Peripheral vascular surgery and magnetic resonance arteriography--a review. Eur J Vasc Endovasc Surg 2001;22(5):396-402.

7. Pruessmann KP, Weiger $M$, Scheidegger MB, Boesiger $P$. SENSE: sensitivity encoding for fast MRI. Magn ResonMed 1999;42(5):952-962.

8. Kurihara $Y$, Yakushiji $Y K_{x}$ Tani I, Nakajima $Y$, Van Cauteren $M$. Coll sensitivity encoding in $M R$ imaging: advantages and disadvantages in clinical practice. AJR Am $J$ Roentgenol 2002; 178(5): 1087-1091.

9. Prince MR, Chabra $S G$, Watts $R$, et al. Contrast material travel times in patients undergoing peripheral MR angiography. Radiology 2002;224(1):55-61.

10. Meissner OA, Rieger $J$, Weber $C$, et al. Critical Limb Ischemia: Hybrid MR Angiography Compared with DSA. Radiology 2005.

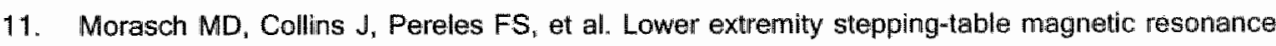
angiography with multilevel contrast timing and segmented contrast infusion. J Vasc Surg 2003;37(1):52-71.

12. Chetter $\| C$, Dolan P, Spark لJll, Scott DJ, Kester RC. Correlating clinical indicators of lower-limb ischaemia with quality of life. Cardiovasc Surg 1997;5(4):361-366.

13. de Vries $S$, Kuipers $W$. Hunink M. Intermittent claudication: Symptom severity versus health values. Joumal of Vascular Surgery 1998;27(3):422-430.

14. Fowkes $F G$, Gillespie $\| N$. Angioplasty (versus non surgical management) for intermittent claudication. Cochrane Database Syst Rev 2000(2):CD000017.

15. Hiatt WR, Hirsch AT, Regensteiner JG, Brass EP. Clinical Trials for Claudication: Assessment of Exercise Performance, Functional Status, and Clinical End Points. Circulation 1995;92(3):614-621.

16. Bosch $\mathrm{JL}$, van der Graaf $Y$, Hunink MG. Health-related quality of lite after angioplasty and stent placement in patients with iliac artery occlusive disease: results of a randomized controlled clinical trial. The Dutch lliac Stent Trial Study Group. Circulation 1999;99(24):3155-3160.

17. Cassar K, Bachoo P, Brittenden J. The Effect of Peripheral Percutaneous Transluminal Angioplasty on Quality of Life in Patients with Intermittent Claudication. European Journal of Vascular and Endováscular Surgery 2003;26(2):130-136.

18. Chetter IC, Spark Ji, Kent PJ, Berridge DC, Scott DJ, Kester RC. Percutaneous transluminal angioplasty for intermittent claudication: evidence on which to base the medicine. Eur J Vasc Endovasc Surg 1998;16(6):477-484. 
19. Domandy JA, Rutherford RB. Management of peripheral arterial disease (PAD). TASC Working Group. TransAtlantic inter-Society Concensus (TASC). J Vasc Surg 2000;34(1 Pt 2):S1-S296.

20. Mehta $T$, Venkata Subramaniam $A$, Chetter 1 , McCollum $P$. Disease-specific quality of life assessment in intermittent claudicationt review. Eur J Vasc Endovasc Surg 2003;25(3):202-208.

21. Spengel $F$, Clement D, Boccalon $H$, Liard $F$, Brown $T$, Lehert P. Findings of the Naftidrofuryl in Quality of Life (NIOOL) European study program. Int Angiol 2002;21(1):20-27.

22. Brenner DJ, Elliston $\mathrm{CD}$. Estimated radiation risks potentially associated with full-body $\mathrm{CT}$ screening. Radiology 2004;232(3):735-738.

23. Koelemay MJ, Legemate DA, van Gurp J, Ponson $A E$, Reekers JA, Jacobs MJ. Colour duplex scanning and pulse-generated run-off for assessment of popliteal and cruropedal arteries before peripheral bypass surgery. Br J Surg 1997;84(8):1115-1119.

24. Koelemay $M J_{;}$Legemate $D A$, de $\operatorname{Vos} H$, et al. Duplex scanning allows selective use of arteriography in the management of patients with severe lower leg arterial disease. J Vasc Surg 2001:34(4):661-667.

25. Grassbaugh JA, Nelson PR, Rzucidlo EM, et al. Blinded comparison of preoperative duplex ultrasound scanning and contrast arteriography for planning revascularization at the level of the tibia. J Vasc Surg 2003;37(6):1186-1190.

26. Leiner $T$, Kessels $A G$, Schurink $G W$, et al. Comparison of contrast-enhanced magnetic resonance angiography and digital subtraction angiography in patients with chronic critical ischemia and tissue loss. Invest Radiol 2004:39(7):435-444.

27. Pellerin $M_{i}$ Coquille $F$, Hubert $M$, Lagrange $C$, Piquois $A$, Scherrer $A$. lComparison between arteriography and magnetic resonance angiography in patients with leg peripheral arterial disease |] J Radiol 2001;82(3 Pt 1):237-243.

28. Portugaller $H R$, Schoellnast $H$, Hausegger $K A$, Tiesenhausen $K$, Amann $W$, Berghold $A$. Multislice spiral $C T$ angiography in peripheral arterial occlusive disease: a valuable tool in detecting significant arterial lumen narrowing? Eur Radiol 2004;14(9):1681-1687.

29. Lawler LP, Fishman EK. Multidetector row computed tomography of the aorta and peripheral arteries. Cardiol Clin 2003;21(4):607-629.

30. Jansen $R$, de Vries $S$, Cullen $K$, Donaldson $M_{n}$ Hunink $M$. Cost-identification analysis of revascularization procedures on patients with peripheral arterial occlusive disease. Journal of Vascular Surgery 1998;28(4):617-623.

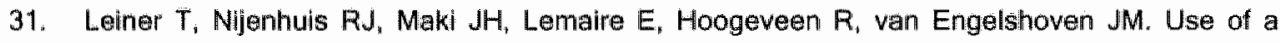
three-station phased array coll to improve peripheral contrast-enhanced magnetic resonance angiography. J Magn Reson Imaging 2004;20(3):417-425.

32. Bezoojien $R$, van den Bosch $H C$, Tielbeek $A V_{\text {, }}$ et al. Peripheral arterial disease: sensitivityencoded multiposition MR anglography compared with intraarterial angiography and conwentional multiposition MR angiography. Radiology 2004;231(1):263-271.

33. Goyen M, Lauenstein TC. Herborn CU, Debatin JF, Bosk S, Ruehm SG. 0.5 M Gd chelate (Magnevist) versus $1.0 \mathrm{M}$ Gd chelate (Gadovist): dose-independent effect on image quality of pelvic three-dimensional MR-anglography. J Magn Reson Imaging 2001;14(5):602-607.

34. Macari $M$, Bini EJ, Xue $X$, et al. Colorectal neoplasms: prospective comparison of thin-section low-dose multi-detector row CT colonography and conventional colonoscopy for detection. Radiology 2002,224(2);383-392.

35. wan Straten $M$, Venema $H W$, Streekstra GJ, Majoie $C B$, den Heeten GJ, Grimbergen $C A$. Removal of bone in CT angiography of the cervical arteries by piecewise matched mask bone elimination. Med Phys 2004;31(10):2924-2933. 


\section{Chapter 9}

Summary and Conclusions

Nederlandse samenvatting

Appendix

Dankwoord

Curriculum Vitae 


\section{Summary and Conclusions}

In the everyday imaging work-up for patients with peripheral arterial disease (PAD), a question arises: Which imaging test is optimal for treatment planning, and at the same time, which is the most costeffective? To address both issues, we have optimized the CE-MRA protocol for patients with PAD, and performed a multicenter cost-effectiveness study: the Diagnostic Imaging for Peripheral Arterial Disease (DIPAD) study.

\section{Optimizing CE-MRA}

Chapter 2 describes a parallel imaging technique (SENSE) in order to optimize peripheral CE-MRA of the legs. This technique (SENSE) reduces the scan time by simultaneously collecting MR signals from multiple reception coils. We used SENSE in this study in combination with a three-station dedicatted surface coil. A total of 19 patients underwent peripheral CE-MRA with SENSE. The findings for these patients were compared to those for a similar patient group that underwent peripheral $C E$ MRA without SENSE. The X-ray angiography was the standard of reference in both groups. The results showed that using SENSE improved vessel-to-background contrast $(P \leq 0.001)$ and enlarged the anatomical coverage, thereby depicting the pedal arch routinely. We conclude that CE-MRA using SENSE facilitates routine, high-quality depiction of the entire peripheral vascular tree of the legs including the pedal arch.

\section{Quality of life}

Assessing the quality of life has become an important outcome measure for the evaluation of patients with PAD. Chapter 3 considers whether disease-specific or generic questionnaires are preferable for assessing the quality of life of patients with $\mathrm{PAD}$. For this purpose, two generic questionnaires [EuroQol-5D and the Short Form 36 (SF-36)] and a disease-specific questionnaire (the VascuQol) were compared to parameters of disease severity such as the Rutherford classification and walking distance. At baseline, 443 patients completed their questionnaires, as did 386 patients after 6 months of follow-up. The results showed that change in disease severity after therapy was better detected by the VascuQol than by the SF-36 or EuroQol-5D. Therefore, the VascuQol is the preferred questionnaire for assessing quality of life in future trials and clinical follow-up of patients with PAD.

DUS, CE-MRA en CTA for the initial imaging workup

Chapter 4 presents the clinical and economic consequences of replacing DUS by CE-MRA for the initial imaging work-up of patients with PAD as part of the DIPAD study. In this prospective multicenter study, 357 patients were randomized to DUS or CE-MRA. All costs of both initial and additional imaging, therapeutic interventions and outpatient visits were collected at each participating hospital during a 6-month 
follow-up period. Other outcome measures were therapeutic confidence of physicians, change in disease severity and change in quality of life.

At the 6-month follow-up, data for 352 patients were analyzed. While CE-MRA and DUS performed equally well in the initial imaging work-up for patients with $P A D$, the two imaging modalities had different qualities that count in their favor. Use of CEMRA resulted in higher therapeutic confidence and reduced the number of additional vascular imaging procedures by $42 \%$ compared to DUS. However, the total diagnostic costs of CE-MRA were $€ 167$ higher than the costs of DUS $(P<0.001)$. No significant differences between DUS and CE-MRA were found for total costs, change in disease severity and change in quality of life (all $P>0.05$ ).

Based on our analyses, we conclude that the choice between DUS and CEMRA for initial imaging work-up for patients with PAD depends on the local expertise with these techniques and cost considerations relevant to the local setting: Centers that seek to improve their imaging work-ups might opt to expand their expertise in CE-MRA rather than DUS, since CE-MRA increases the physician's confidence in taking treatment decisions, which reduces the need for additional imaging.

Chapter 5 presents the overall results of the DIPAD study, which compares DUS, CE-MRA, and CTA for the initial imaging work-up for patients with PAD. Patients were randomly assigned to undergo the CE-MRA or the currently employed test, which was CTA in one hospital (Rotterdam) and DUS in the other three hospitals. For this purpose, we included the data of the group of 157 patients that were randomized to CE-MRA or CTA with the data of the group of 357 patients that were randomized to CE-MRA or DUS. At the 6-month follow-up, 508 patients were analyzed. There were no significant differences in improvement of disease severity or quality of life among the CTA, CE-MRA, and DUS groups. The use of both CTA and CE-MRA resulted in less need for additional vascular imaging tests and more confidence in treatment decisions the use of DUS. The CTA led to cost savings compared to both CE-MRA and DUS in the initial imaging evaluation of patients with PAD.

\section{Future directions of CE-MRA}

Chapter 6 reports our preliminary experience with peripheral CE-MRA on a $3.0 \mathrm{~T}$ whole body scanner with a standard built-in body coil. Four healthy volunteers were imaged on the 3.0 T scanner, two of whom were also imaged on a $1.5 \mathrm{~T}$ scanner for comparative purposes. The results show that peripheral CE-MRA on $3.0 \mathrm{~T}$ is feasible and that the vessel-to-background contrast and subjective image quality of the popliteal and lower leg arteries are better than those of imaging on a $1.5 \mathrm{~T}$ scanner.

Chapter 7 assesses the CE-MRA data regarding accuracy-of-stenosis measurements of aortoiliac arteries when a semi-automated software program is used, and compares the accuracy of these measurements with the accuracy of conventional stenosis measurements. In this prospective study, 25 patients underwent CE-MRA and X-ray angiography. Three observers took the semi- 
automated measurements independently, and three other observers took the conventional stenosis measurements independently. Semi-automated measurements had the same high sensitivity and specificity values as conventional stenosis measurements. There was good interobserver agreement among all readers of semi-automated measurements (combined kappa $=0.78$ ) and conventional measurements (combined kappa $=0.83$ ). Therefore, we conclude that semi-automated analysis of aortoiliac $3 D$ CE-MRA has the same high accuracy for detecting stenosis as conventional readings of CE-MRA.

Chapter 8 provides a general discussion that places the results of our study in a clinical perspective. Recommendations to choose among DUS, CE-MRA, or CTA are based on the results of our cost-effectiveness study. Finally, we describe the recent developments that may further improve the accuracy and use of CE-MRA in everyday clinical practice.

\section{Conclusions of this thesis}

1. The use of a parallel imaging technique optimizes peripheral CE-MRA in terms of improving vessel-to-background contrast and enlarging the anatomical coverage, thereby depicting the pedal arch routinely:

2. The disease-specific questionnaire (VascuQol) is preferred to generic questionnaires (SF-36 and EuroQol-5D) in order to assess quality of life in future trials and clinicall follow-up of patients with PAD.

3. The choice of DUS, CE-MRA, or CTA for the initial imaging work-up of patients with PAD depends on the local expertise with these techniques and cost considerations relevant to the local setting. Centers that seek to improve their imaging work-up might opt to expand their expertise of CE-MRA (rather than DUS or CTA), as the clinical value of CE-MRA outweighs that of DUS, and the patient is not exposed to the radiation and nephrotoxic contrast agents that CTA requires.

4. Peripheral CE-MRA on $3.0 \mathrm{~T}$ is feasible and provides better image quality than imaging on $1.5 \mathrm{~T}$.

5. Semi-automated analysis of aortolliac 3D CE-MRA has the same high accuracy for detecting stenosis as conventional readings of CE-MRA. 


\section{Samenvatting}

\section{Diagnostische tests voor perifeer arterieel vaatlijden}

Perifeer arterieel vaatlijden (PAV) is een vaak voorkomende en veelal invaliderende ziekte, die met name voorkomt bij oudere rakende mannen. De ziekte is een gevolg van atherosclerose (aderverkalking) waardoor de beenslagaders vernauwen en op den duur zelfs verstopt raken. Als gevolg hiervan krijgt de patiënt klachten variërend van pijn bij het lopen tot niet genezende wonden aan de benen. De vaatvernauwingen kunnen worden behandeld door het oprekken van de bloedvaten, het z.g. dotteren, of met een bypass operatie.

Bij het opstellen van een behandelplan is beeldvorming nodig om de ernst en de plaats van de vernauwingen nauwkeurig in beeld te brengen. Röntgenangiografie wordt van oudsher beschouwd als het meest betrouwbare onderzoek voor het opsporen van deze vaatvernauwingen. Dit onderzoek is echter belastend voor de patiënt, aangezien het een invasieve techniek betreft, waarbij een katheter rechtstreeks in de beenslagaders van de patiënt moet worden geplaatst en een opname in het ziekenhuis vaak noodzakelijk is. Het is dan ook logisch dat de laatste jaren meer en meer gebruik wordt gemaakt van andere niet invasieve technieken, zoals de contrast versterkte ("enhanced") magnetische resonantie angiografie (CEMRA), echografie (duplex ultrasound (DUS), of de computer tomografische angiografie (CTA). Het is echter zaak die beeldvormende techniek te kiezen die het meest optimaal is voor het opstellen van een behandelplan, maar die ook het meest kosteneffectief en voor de patiënt het minst belastend is.

In dit proefschrift wordt onderzocht welke beeldvormende techniek het beste gebruikt kan worden bij patiënten met perifeer arterieel vaatlijden. Dit gebeurt door een grote groep patiënten door middel van randomisatie voor éen van deze technieken in aanmerking te laten komen en vervolgens de kosten en effecten van diagnostiek en behandeling gedurende het daarop volgend half jaar te vergelijken. Voor dit doel werd de Diagnostic Imaging of Peripheral Arterial Disease (DIPAD) studie opgezet, een multicenter studie waarin 3 academische en 1 perifeer ziekenhuis participeerden. Bovendien worden in dit proefschrift enkele mogelijke verbeteringen van de CE-MRA techniek onderzocht.

\section{Optimalisatie van de CE-MRA}

Het uitvoeren van perifere CE-MRA vereist slechts één enkele injectie van een contrastmiddel waardoor achtereenvolgens de slagaders in het bekken, boven- en onderbenen worden afgebeeld. Daarbij is het van belang dat er snel wordt gescand om te voorkomen dat ook de aders (venen) in het onderbeen aankleuren, hetgeen de beoordeling van de slagaders bemoeilijkt. Aan de andere kant kan ook niet te snel worden gescand, omdat dit ten koste gaat van het detail en het signaal in de bloedvaten.

In hoofdstuk 2 wordt een nieuwe techniek voor de verdere verbetering van perifere CE-MRA beschreven. Bij het gebruik van deze nieuwe techniek, parallel imaging of SENSE genoemd, detecteren meerdere ontvangstspoelen tegelijkertijd 
hel signaal waardoor ofwel sneller gescand kan worden, ofwel beelden met meer detail en /of signaal gemaakt kunnen worden. Om aan te tonen dat deze SENSE techniek in de praktijk ook de beoogde verbeteringen oplevert zijn 19 achtereenvolgende patiênten met PAV door middel van CE-MRA zonder SENSE en daarna 19 PAV patienten met SENSE onderzocht. De verkregen CE-MRA beelden werden onderling en met de resultaten van röntgen-angiografie (de gouden standaard) vergeleken. De resultaten van deze studie tonen aan dat de combinatie van CE-MRA met SENSE de beeldkwaliteit (vaat - achtergrond contrast) van de bekken en beenslagaders significant verbetert. Bovendien kan vanwege de versnelde acquisitie een groter anatomisch bereik worden gescand, waardoor het mogelijk is geworden om bij alle patiënten de arteriële voetboog af te beelden.

\section{Kwaliteit van leven}

Of een behandeling effect heeft wordt meestal beoordeeld aan de hand van technische objectieve parameters zoals bijvoorbeeld het bepalen van "de enkel-arm index": Deze parameters blijken echter de subjectieve beleving van de patiënt onnauwkeurig weer te geven en blijft het de vraag of de kwaliteit van leven van de patiènt na de behandeling is verbeterd.

Om de kwaliteit van leven in kaart te brengen zijn speciale vragenlijsten ontwikkeldl. Tot op heden werden vooral algemene vragenlijsten gebruikt waarmee de kwaliteit van leven bij patiënten met uiteenlopende aandoeningen kan worden bepaald. Recent is een specifieke vragenlijst ontwikkeld, speciaal bestemd voor patiënten met PAV. Tot op heden is het echter onduidelijk of deze specifieke vragenlijst te verkiezen is boven de algemene vragenlijsten bij het bepalen van de kwaliteit van leven bij patiënten met PAV. In hoofdstuk 3 wordlen de resultaten van de specifieke vragenlijsten (de VascuQol) en de 2 algemene vragenllijsten (de Short Form-36 (SF-36) en de EuroQol-5D) vergeleken met frequent gebruikte ziekteparameters zoals de Rutherford classificatie en de loopafstand. Hiertoe hebben aan het begin van de DIPAD studie 443 patiënten, en na 6 maanden followup 386 patiènten, deze vragenlijsten ingevuld en teruggestuurd. Analyse van de ingevulde vragenlijsten heeft aangetoond dat de specifieke vragenlijst (de VascuQol) beter de verandering kwaliteit van leven in deze patiëntengroep kan detecteren dan de algemene vragenlijsten (SF-36 en de EuroQol-5D). De VascuQol verdient derhalve de voorkeur bij het bepalen van de kwaliteit van leven van patiënten met PAV.

\section{DUS, CE-MRA en CTA voor de diagnostiek van perifeer vaatlliden}

De DIPAD studie was opgezet om te onderzoeken met welke beeldvormende techniek (CE-MRA, CTA of DUS) de ernst en uitgebreidheid van perifeer arterieel vaatlijden het beste bepaald kon worden alvorens te beslissen welke behandeling het meest aangewezen was. Deze studie werd uitgevoerd in één niet academisch ziekenhuis en in drie academische ziekenhuizen. In elk deelnemend centrum werd gerandomiseerd tussen de CE-MRA en de in dat ziekenhuis gangbare 
beeldvormende test. In één ziekenhuis was de gangbare test de CTA (Rotterdam). en voor de overige 3 ziekenhuizen was dit DUS.

In hoofolstuk 4 worden de resultaten gepresenteerd van 357 patienten met perifeer arterieel vaatlijden die werden gerandomiseerd tussen CE-MRA en DUS. Alle kosten die nodig waren voor (additionele) beeldworming, behandeling en polibezoeken werden gedurende 6 maanden verzameld. Daarnaast werd gekeken naar de zekerheid van de specialist om een therapeutische beleidsbeslissing te nemen na het uitgevoerde beeldvormend onderzoek, en naar de verandering in de ernst van de ziekte, en verandering in kwaliteit van leven.

$\mathrm{Na} 6$ maanden follow-up konden de gegevens van 352 patiènten worden geanalyseerd. Als eerste beeldvormende test voor de diagnostische work-up van patiënten met PAV bleken CE-MRA en DUS niet voor elkaar onder te doen, met dien verstande dat beide diagnostische testen specifieke voordelen hadden ten opzichte van de ander. De klinische bruikbaarheid van de CE-MRA was beter dan van DUS, omdat de mate waarin de specialist zich zeker voelde om een beleidsbeslissing te nemen groter was na CE-MRA dan na DUS. Het aantal additionele beeldvormende onderzoeken na CE-MRA was aanzienlijk minder ( $42 \%$ ) dan na DUS. Na 6 maanden follow-up verschilden beide groepen patiënten echter niet wat betreft hun functionele parameters (Rutherford classificatie, enkel-arm index en loopafstand) en kwaliteit van leven. De totale diagnostische kosten voor alle beeldvormende onderzoeken tezamen waren gemiddeld $€ 167$ hoger in de CE-MRA groep dan in de DUS groep. Dit verschil was statistisch significant.

De resultaten van deze studie laten zien dal de keuze tussen DUS en CEMRA als eerste beeldvormende test voor de diagnostische work-up bij patiënten met PAV zal afhangen van de lokale ervaring en beschikbaarheid van deze technieken, daarbij rekening houdend met de lokale kostenoverwegingen. Ziekenhuizen die hum diagnostische work-up willen verbeteren kunnen beter voor CE-MRA dan voor DUS kiezen, omdat de clinicus zich na CE-MRA zekerder voelt bij het nemen van een therapeutische beslissing en bovendien na CE-MRA beduidend minder behoefte blijkt te hebben aan aanvullend beeldonderzoek.

In hoofdstuk 5 worden de overall resultaten van de DIPAD studie gepresenteerd, waarbij het gebruik van DUS, CE-MRA of CTA als eerste beeldvormende test bij patiënten met PAV met elkaar worden vergeleken. De groep van 357 patiënten waarbij werd gerandomiseerd tussen CE-MRA en DUS werd voor dit doel aangevuld met een groep van 157 patiënten waarin werd gerandomiseerd tussen CE-MRA en CTA. Na 6 maandien konden de gegevens van 508 patiênten geanalyseerd worden. Daarbij bleek dat de verbeteringen in functionele parameters (Rutherford classificatie, enkel-arm index en loopafstand) en kwaliteit van leven tussen de patiënten in de CTA, CE-MRA en DUS groep niet verschilden. De totale diagnostische kosten in CTA en DUS groep waren echter significant lager dan de kosten in CE-MRA groep. Echter patiënten gerandomiseerd voor CTA en CE-MRA hadden minder additionele beeldvormende onderzoeken ondergaan, en de mate waarin de specialist zich zeker voelde om een beleidsbeslissing te nemen was groter in de CE-MRA en CTA groep dan in de DUS groep. 
Overeenstemmend met hoofdstuk 4 kan op basis van deze analyses worden geconcludeerd dat zowel CE-MRA als CTA de clinicus meer zekerheid verschaffen dan DUS bij het opstellen van een behandelplan voor patienten met perifeer arterieel vaatlijden. Hierdoor wordt na CE-MRA en CTA beduidend minder vaak aanvullende beelddiagnostiek aangevraagd. Het gebruik van CTA als initiële test leidt, in vergelijking met CE-MRA en DUS, mogelijk zelfs tot enige kostenbesparing.

\section{Nieuwe ontwikkelingen voor de CE-MRA}

In hoofdstuk 6 beschrijven we onze eerste ervaring met perifere CE-MRA uitgevoerd op een scanner met een 2 keer zo sterk magneetveld (3.0 Tesla). Scannen met een sterker magneetveld heeft het voordeel dat de signaal/ruis verhouding in de bloedvaten verbetert. Bij vier gezonde vrijwilligers werd een perifere CE-MRA verricht op een 3.0 T scanner, waarna de resultaten werden vergeleken met de beelden die verkregen werden bij perifere CE-MRA op een $1.5 \mathrm{~T}$ scanner uitgevoerd bij 2 van deze 4 vrijwilligers. De resultaten laten zien dat perifere CE-MRA succesvol kan worden uitgevoerd op een 3.0 T scanner, en dat de beeldkwaliteit van de slagaders ter hoogte van knieën en onderbenen wordt verbeterd ten opzichte van de $1.5 \mathrm{~T}$ scanner.

Een nadeel van de CE-MRA is dat de beoordeling en bewerking van de beeldinformatie tijdrovend en daardoor duur is. Recent is een software programma ontwikkeld dat semi-automatisch de ernst en lokalisatie van de vernauwingen in het bloedvat in CE-MRA data bepaalt. In hoofdstuk 7 wordt de nauwkeurigheid bepaald van dit software programma. In deze prospectieve studie ondergingen 25 patiënten met PAV een CE-MRA en een röntgen-angiografisch onderzoek. Drie onafhankelijke beoordeelaars bepaalden de mate van vernauwingen in de bekkenslagaders met behulp van dit semi-automatische software programma. Deze resultaten werden vergeleken met drie andere onafhankelijke beoordeelaars die de vernauwingen in de CE-MRA beelden op de traditionele manier bepaalden.

De semi-automatische metingen hadden een vergelijkbare sensitiviteit en specificiteit als de traditionele metingen. Ook was de tijdsduur en de mate van interobserver variatie bij semi-automatische metingen (gecombineerde kappa=0.78) vrijwel gelijk aan traditionele metingen (gecombineerde kappa $=0.83$ ). Daarom werd geconcludeerd dat op CE-MRA beelden het semi-automatische software programma net zo snel en nauwkeurig de ernst en lokalisatie van vernauwingen in de bekkenslagaders kan bepalen als de traditionele metingen.

In hoofdstuk 8 worden de bevindingen van dit proefschrift in een klinisch perspectief geplaatst. Op basis van de resultaten van onze DIPAD studie geven we aanbevelingen hoe ieder voor zich tussen de DUS, CE-MRA en CTA moet kiezen. Tenslotte, bespreken we de laatste ontwikkelingen van de perifere CE-MRA. 


\section{Conclusies}

De conclusies van dit proefischrift zijn:

1. Indien bij perifere CE-MRA gebruik gemaakt wordt van een parallel imaging technilek (SENSE) verbetert de signaal ruis verhouding en komt altijd de hele perifere vaatboom inclusief de arteriële voetboog goed in beeld.

2. Ten behoeve van het bepalen van de kwaliteit van leven bij patienten met perifeer arterieel vaatlijden verdient de specifieke vragenlijst (de VascuQol) de voorkeur boven de algemene vragenlijsten (de SF-36 en de EuroQol-5D).

3. De keuze tussen DUS, CE-MRA en CTA in de diagnostische work-up van patiënten met perifeer arterieel vaatlijden moet worden gemaakt op basis van de lokale ervaring en beschilkbaarheid van deze technieken, daarbij rekening gehouden met de lokale kostenoverwegingen. CTA is daarbij goedkoper dan CE-MRA en DUS. Ziekenhuizen die de diagnostische work-up willen verbeteren kunnen beter CE-MRA kiezen, omdat de klinische bruikbaarheid beter is dan van DUS én er is geen risico op contrastnephropathie of stralingsbelasting zoals bij CTA.

4. Perifere CE-MRA kan succesvol, en met een verbeterde beeldkwaliteit, worden uitgevoerd op de $3.0 \mathrm{~T}$ scanner.

5. Een semi-automatische software programma is even nauwkeurig als een subjectieve waarnemer bij het beoordelen van de ernst en lokalisatie van vernauwingen van bekkenslagaders op CE MRA beelden. 


\section{Appendix}

Centers that participated in the Diagnostic Imaging for Peripheral Arterial Disease: study:

Academisch Ziekenhuis Maastricht

M.W. de Haan, J.M.A. van Engelshoven, K. Flobbe, P. Habets, E. Lemaire (Radiology)

P.J.E.H.M. Kitslaar, J.H.M. Tordoir, G.W.H. Schurink, M.J.H.M. Jacobs (Vascular Surgery)

A.G. Kessels, P.J. Nelemans (Epidemiology)

M. Nullens (Vascular laboratory)

\section{Erasmus Universitair Medisch Centrum Rotterdam}

M.G.M. Hunink, R.Ouwendijk P.M.T. Pattynama, M.C.J.M. Kock, W.J. van Leeuwen. C.H. van Bavel, M. Dijkshoorn, L. Everse, H. Muharam-Kamminga (Radiology)

H. van Urk, M.R.H.M. van Sambeek (Vascular Surgery)

Catharina Ziekenhuis Eindhoven

A.V. Tlelbeek, L.E.M Duym, J. Schut (Radiology)

J Buth, P.W.M. Cuypers, A Schouten-Saarloos (Vascular Surgery)

A. Versteeg (Vascular laboratory)

Universitair Medisch Centrum St Radboud, Nijmegen

J.O. Barentsz, F.M.J. Heijstraten, L.J. Schultze Kool, M.P. van Proosdij, K. Powell, M. Hogenkamp, S. Kleuskens, M. Verhoeven (Radiology)

J.A. van der Vliet, P.L. Klemm, H.C.J.L. Buscher, M. van Dam, (Vascular Surgery)

S. Holewijn (Vascular laboratory) 


\section{Dankwoord}

Eindelijk klaar! Wat in het begin allemaal zo ver wegleek is nu afgerond to dit proefschrift. Door de jaren heen hebben heel veel mensen mij enthousiast met dit proefschrift en de DIPAD studie geholpen. I $\mathrm{k}$ wil iedereen hiervoor bedanken, en met name de volgende personen.

De DIPAD harde kern, waarbij ik eerst professor Jos van Engelshoven en protessor Myriam Hunink, mijn promotors wil bedanken voor hun goede adviezen, Michiel de Haan, erg waardevol waren jouw praktische inzichten. Patty Nelemans, ik vond Jouw kritische blik heel erg verhelderend, dit heeft me enorm geholpen om alles te kunnen afronden. Fons Kessels, ik wond jouw continue betrokkenheid bij alle projecten erg bewonderenswaardig. Karin Flobbe, jij hebt me vooral in het begin wegwijs gemaakt in de onderzoekswereld, dankjewel. Peggy Habets, heel erg bedankt voor de nauwkeurige invoer van alle data, dit was echt een enorme klus. En daarbij ook Wibeke van Leeuwen en Caroline van Bavel uit Rotterdam, zij hebben alle data van de vragenlijsten verwerkt. Rody Ouwendijk, ik vond het fijn om met jou de DIPAD studie te voltooien, succes met je eigen promotie!

Verder will ik alle vaatchirurgen, overige betrokken radiologen, MR laboranten en het vaatlab uit Maastricht bedanken voor het eindeloos invullen van vele formulieren.

Het leuke aan de DIPAD studie was dat je regelmatig op bezoek moest bij éën van de deelnemende centra. Ik vond het erg plezierig om overal eens rond te kijken. Hierbij wil ik dan ook alle betrokken radiologen, vaatchirurgen, het vaatlab, röntgenlaboranten uit Universitair Medisch Centrum St Radboud Nijmegen, Catharina Ziekenhuis Eindhoven en Erasmus Universitair Medisch Centrum Rotterdam bedanken voor hun inzet in de DIPAD studie. Marijke Hogenkamp jij hebt de studie in Nijmegen draaiende gehouden, (jij weet hard werken en veel kletsen uitstekend te combineren).

Tim Leiner, altijd weer verras en help je mij met het uitwerken van nieuwe onderzoeksideeën, thanks! Ine Kengen bedankt voor het uitlijnen van het proefschrift. Verder wil ik langs deze weg vooral mijn familie en vrienden bedanken voor hun support tijdens de periode van dit onderzoek.

Beste kamergenootjes, bedankt voor jullie geduld met mij, het was erg gezellig om met jullie te geinen. Dus Suzanne, Nils, Robbert, Dave en Vera, heel veel succes met jullie eigen promotie's en ik hoop dat er nog vele zeill, fiets, langlauf, borrel festijnen volgen!

Tot slot lieve Quido, jouw enthousiasme voor onderzoek heeft aanstekelijk gewerkt. Ik hoop dat we in de opleiding net zo veel lol hebben als we deze jaren als onderzoeker hebben gehad! 


\section{Curriculum vitae}

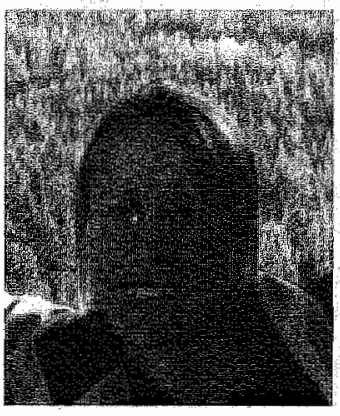

Marianne de Vries was born on June 21, 1976 in Groningen, The Netherlands. She moved to Arnhem were she obtained her high school diploma at the Catholic Gelders Lyceum in 1994.

In the same year she started Medical School at Leuven Catholic University in Leuven, Belgium. In 1995 she worked as a nurse in the Sana Clinic in Munich, Germany. During the internships she worked for several months with a general practitioner in Luxembourg.

In 2001 she received her MSc degree in Medicine with honors, and she finished her one year trainee as general practitioner. In 2001 she worked for several months as a resident on the Emergency Room in TweeSteden Ziekenhuis in Waalwijk, The Netherlands.

In October 2001 she became a research fellow at the Department of Radiology of Maastricht University Hospital. The results of this research are presented in this thesis. Beginning 2005, she started her Radiology residency in Maastricht. 
